\title{
A PROOF OF PROJECTIVE DETERMINACY
}

\author{
DONALD A. MARTIN AND JOHN R. STEEL
}

\section{INTRODUCTION}

Let $\omega$ be the set of all natural numbers. ${ }^{\omega} \omega$ is the set of all functions from $\omega$ to $\omega$. We regard ${ }^{\omega} \omega$ as a topological space by giving it the product of the discrete topology. (With this topology it is homeomorphic to the irrationals.) A subset of the finite product ${ }^{j}\left({ }^{\omega} \omega\right)$ is projective if it comes from an open subset of some ${ }^{k}\left({ }^{\omega} \omega\right)$ via repeated applications of projection and complementation. The projective sets admit a natural hierarchy: a set is $\Sigma_{0}^{1}$ iff it is open, $\Pi_{n}^{1}$ iff it is the complement of a $\Sigma_{n}^{1}$ set, and $\Sigma_{n+1}^{1}$ iff it is the projection of a $\Pi_{n}^{1}$ set.

Although the projective sets are in some sense simply definable, many questions concerning them cannot be answered within ZFC, the standard system of axioms for set theory. In general, ZFC gives a good theory of Borel sets, decides some questions concerning $\Pi_{1}^{1}$ and $\Pi_{2}^{1}$ sets, and decides little about $\Pi_{3}^{1}$ and beyond. For example, while ZFC proves that all $\Pi_{1}^{1}$ sets are Lebesgue measurable (Lusin, cf. [12, p. 105]), it neither proves nor refutes the assertion that all $\Pi_{2}^{1}$ sets are Lebesgue measurable (Gödel, Solovay; cf. [3, pp. 528, 537]). The situation is similar with regard to other "regularity" properties of projective sets, for example the Baire property and the property of either being countable or containing a perfect subset. In a different direction, ZFC proves that all $\Pi_{1}^{1}$ relations admit $\Pi_{1}^{1}$ selection functions (Novikov, Kondo; cf. [12, p. 227]), but does not decide whether all $\boldsymbol{\Pi}_{2}^{1}$ relations admit projective selection functions (Levy [6]).

There is, nevertheless, a fundamental regularity hypothesis concerning projective sets, one which seems to decide all of the open questions about them. To each set $Y$ and each $A \subseteq{ }^{\omega} Y$ is associated a two-person infinite game played

Presented at the Special Session on Determinacy and Large Cardinals at the Annual Meeting of the American Mathematical Society in New Orleans, January 1986; received by the editors April 21,1988

1980 Mathematics Subject Classification (1985 Revision). Primary 03E15, 03E55; Secondary $03 \mathrm{E} 60$.

The authors would like to give special thanks to the referees, whose lists of corrections and suggestions were extraordinary in both length and quality.

The authors were partially supported by NSF Grant DMS84-02983.

(C)1989 American Mathematical Society 
as follows:

$$
\begin{array}{llllll}
I & y_{0} & & y_{2} & & \ldots \\
I I & & y_{1} & & y_{3} & \cdots
\end{array}
$$

Each $y_{i}$ must belong to $Y . I$ wins a play of the game if and only if $\left\langle y_{i}\right| i \in$ $\omega\rangle \in A$. The notions of strategy and winning strategy for the game associated with $A$ (and $Y$ ) are defined in the natural way. $A$ is determined (as a subset of ${ }^{\omega} Y$ ) if one of the two players has a winning strategy for the associated game. $\Pi_{n}^{1}$ determinacy is the assertion that all $\Pi_{n}^{1}$ subsets of ${ }^{\omega} \omega$ are determined; projective determinacy, or PD, is the assertion that all projective subsets of ${ }^{\omega} \omega$ are determined.

During the last 25 years or so, descriptive set theorists have shown that PD settles all the classical questions about projective sets left undecided by ZFC.

The earliest results concerned the regularity properties: PD implies all projective sets are Lebesgue measurable (Mycielski, Swierczkowski), have the Baire property (Banach, Mazur), and either are countable or have a perfect subset (Davis) (cf. [12, pp. 295-301]). Later, through the work of Kechris, Moschovakis, and others, it was shown that, under PD, the deepest methods and results of the classical theory can be generalized and extended to the whole of the projective hierarchy. For example, Moschovakis found the proper generalization of the classical sieves on $\Pi_{1}^{1}$ sets (a basic tool for dealing with such sets), and showed that under PD $\boldsymbol{\Pi}_{2 n+1}^{1}$ sets admit such "generalized sieves." As a consequence, $\Pi_{2 n+1}^{1}$ relations admit $\Pi_{2 n+1}^{1}$ selection functions [12, p. 317]. The theory of projective sets one gets from PD generalizes in a natural way the theory of Borel, $\Pi_{1}^{1}$, and $\Pi_{2}^{1}$ sets one gets from ZFC; indeed, with the benefit of hindsight we can regard much of the latter theory as based on $\Pi_{0}^{1}$ determinacy, which is provable in ZFC (Gale and Stewart; cf. [12, p. 289]).

Because of the richness and coherence of its consequences, one would like to derive PD itself from more fundamental principles concerning sets in general, principles whose justification is more direct.

We know of one proper extension of ZFC which is as well justified as ZFC itself, namely ZFC + "ZFC is consistent." Extrapolating wildly, we are led to strong reflection principles, also known as large cardinal hypotheses. (One can fill in some intermediate steps.) These principles assert that certain properties of the universe $V$ of all sets are shared by, or "reflect to," initial segments $V_{\alpha}$ of the cumulative hierarchy of sets. (Reflecting reflection, we get ordinals $\kappa$ such that certain properties of $V_{\kappa}$ reflect to smaller $V_{\alpha}$ 's. This is the form of the principles below.) Reflection principles have some motivation analogous to that for the axioms of ZFC themselves, and indeed the axioms of infinity and replacement of ZFC are equivalent to a reflection schema (Levy, Montague; cf. [5]).

The principles of interest to us assert the existence of nontrivial elementary embeddings $j$ of the universe $V$ into transitive classes $M$. The greater the 
resemblance between $M$ and $V$, the stronger the principle. ( $M=V$ is impossible by a result of Kunen; cf. [3, p. 314]). The critical point of such an embedding $j$ or crit $(j)$, is the least ordinal $\alpha$ such that $j(\alpha) \neq \alpha$. To see the reflection inherent in such a principle, notice that if $P$ is a property of $\kappa=\operatorname{crit}(j)$, and $M$ resembles $V$ enough that $P(\kappa)$ is true in $M$, then $M$ satisfies $(\exists \alpha<j(\kappa)) P(\alpha)$, so that if $j$ is elementary with respect to $P, V$ satisfies $(\exists \alpha<\kappa) P(\alpha)$.

The "large cardinal" associated to an elementary $j: V \rightarrow M$ is its critical point; the more $M$ resembles $V$, the larger this cardinal must be. A cardinal $\kappa$ is measurable iff $\kappa=\operatorname{crit}(j)$ for some nontrivial elementary $j: V \rightarrow M$. A cardinal $\kappa$ is superstrong iff $\kappa=\operatorname{crit}(j)$ for some nontrivial elementary $j: V \rightarrow M$ with $V_{j(\kappa)} \subseteq M$. A cardinal $\kappa$ is $\omega$-huge iff $\kappa=\operatorname{crit}(j)$ for some nontrivial elementary $j: V \rightarrow M$ with $V_{j_{\omega}(\kappa)} \subseteq M$, where $j_{\omega}(\kappa)=$ $\sup \{j(\kappa), j(j(\kappa)), j(j(j(\kappa))), \ldots\}$. By Kunen's result there is no nontrivial elementary $j: V \rightarrow M$ with $V_{j_{\omega}(\kappa)+1} \subseteq M$.

The first indication that large cardinals are connected with determinacy was Solovay's proof (cf. [3, p. 556]) that PD implies there is an inner model with a measurable cardinal. This suggested that large cardinal hypotheses would be necessary in proving determinacy. Results of Gaifman, Rowbottom, and Solovay suggested they might suffice, and indeed in [7] Martin showed that the existence of a measurable cardinal implies $\Pi_{1}^{1}$ determinacy. The next natural target was $\Pi_{2}^{1}$ determinacy. By extending Solovay's arguments, Martin, Simms, and Green showed that small fragments of $\boldsymbol{\Pi}_{2}^{1}$ determinacy imply the existence of inner models with many measurable cardinals. Thus one needed a hypothesis stronger than the existence of many measurables in order to prove $\Pi_{2}^{1}$ determinacy.

During the ten years between [7] and [8] considerable effort was devoted to deriving $\boldsymbol{\Pi}_{2}^{1}$ determinacy from the existence of supercompact cardinals, a hypothesis slightly more powerful than the existence of superstrong cardinals. There was no success. Then Martin [8] showed that the existence of $\omega$-huge cardinals implies $\boldsymbol{\Pi}_{2}^{1}$ determinacy. Although the hypothesis seemed outlandishly strong, the proof looked natural to descriptive set theorists. This and the earlier failure with supercompacts and the like led to a general opinion that the existence of an $\omega$-huge cardinal was a necessary hypothesis for $\Pi_{2}^{1}$ determinacy. This opinion was reinforced by subsequent work of Woodin, who showed that PD (and more) follows from large cardinal hypotheses somewhat stronger than the existence of an $\omega$-huge cardinal. It seemed one had only to wait for the inner model theory to reach these very large cardinals in order actually to prove them necessary for PD. Unfortunately, the inner model theory had bogged down well below superstrongs.

It came as a great surprise when Woodin [13], using freshly minted techniques of Foreman, Magidor, and Shelah [2], showed that the existence of supercompact cardinals implies all projective sets are Lebesgue measurable. This meant 
that if there are inner models with supercompact cardinals which resemble in certain very basic ways the known inner models with measurable cardinals and the like, then these models with supercompacts must be quite complicated-too complicated to construct under the hypothesis of PD. If the pattern of connection between large cardinals and determinacy that was known in detail at the level of $\Pi_{1}^{1}$ determinacy and slightly beyond were to persist, this meant that supercompact cardinals must imply PD. In fact, Woodin and Shelah soon lowered the large cardinal hypothesis for the Lebesgue measurability of projective sets below superstrongs. In particular, Woodin showed that the existence of $n$ "Woodin cardinals" (as they are now known) with a measurable above them all implies that all $\Pi_{n+2}^{1}$ sets are Lebesgue measurable. If the earlier pattern were to persist, this meant that the existence of $n$ Woodin cardinals with a measurable above them all must imply $\Pi_{n+1}^{1}$ determinacy.

In this paper we show that this is in fact the case. Thus the existence of infinitely many Woodin cardinals implies PD. We also give the proof, using a result of Woodin, that every set in $L(\mathscr{R})$ is determined if there are infinitely many Woodin cardinals with a measurable cardinal above them all.

Our large cardinal hypotheses are essentially weakest possible. For the case of $\Pi_{2}^{1}$ determinacy, we show this in [11] by getting an inner model for a Woodin cardinal in which $\Pi_{2}^{1}$ determinacy fails. Similar results can be proved for higher levels of the projective hierarchy. More recently Woodin has shown that, e.g. $\Pi_{2}^{1}$ determinacy implies that there is an inner model for a Woodin cardinal, and he has gotten some equiconsistency results relating determinacy and Woodin cardinals.

As the foregoing capsule history of determinacy proofs suggests, the authors owe a great debt to the work of Foreman, Magidor, Shelah, and Woodin. Nevertheless, the technical ideas in our proof have no relation to that work. We arrived at our proof by asking what it is about Woodin cardinals that makes their inner models so complicated. In earlier work on inner model theory, there had arisen the worry that superstrong cardinals might generate complicated "iteration trees" (cf. §3). It turns out that Woodin cardinals generate such trees, and, while this is a problem for inner model theory, it can be used to prove determinacy. Our proof also makes heavy use of the connection between determinacy and homogeneous trees and of the method for constructing homogeneous trees of [9].

For more on the background of our theorems, as well as condensed versions of some of the proofs, see [10].

We have tried to make this paper fairly self-contained. Thus in $\S 1$ we develop the theory of the ultrapowers given by towers of measures and especially the theory of extenders in some detail, although we are presenting mostly material familiar to large cardinal experts. Similarly, in $\S 2$ we develop the theory of homogeneous trees from the beginning, a theory well known to experts in determinacy. The reader needs: for $\S 1$ a familiarity with axiomatic set theory at the 
level of an introductory graduate course and an acquaintance with measurable cardinals (with the ultrapowers they give and with Rowbottom's Theorem); for $\S 2$ the definition of the projective hierarchy and the normal form for $\Pi_{1}^{1}$ sets in terms of well-orderings. At the end of $\S 2$ we state our Main Theorem, using the concepts introduced in $\S 2$ and the bare definition of Woodin cardinals. We then deduce the determinacy results mentioned above. The rest of the paper is devoted to proving the Main Theorem. In $\S 3$ we introduce and study our principal technical concept: that of iteration trees. In $\S 4$ we develop the theory of Woodin cardinals, relating them to other large cardinals and showing how they can be used to generate iteration trees. The results of $\S \S 3$ and 4 are combined in $\S 5$ to prove the Main Theorem. No new assumptions of knowledge on the part of the reader are made in $\S \S 3-5$.

In order to avoid technicalities that might obscure the intuitive ideas, we have made free use of proper classes. The reader who would like not to think about technicalities but who nevertheless wants to regard the paper as proving results in a definite formal theory should think of Kelley-Morse set theory as the working theory of the paper. With one exception, everything we do obviously makes sense and goes through in KM. (We explain how to deal with the exception when it arises.) Actually all our uses of proper classes could be eliminated, since we need apply our lemmas about proper classes only to certain specific classes, so our main results are provable in ZFC. We shall occasionally make comments about our uses of proper classes.

Our exposition has been influenced by a set of lectures given by Matthew Foreman on our results in 1985. Readers familiar with notes from those lectures should be warned, however, that the proof, the concepts, and the terminology are somewhat changed.

\section{EXTENDERS}

In this section we assemble various concepts and facts from the theory of large cardinals.

Convention. Throughout this paper we shall mean by a measure on a set $X$ a function $\mu: \mathscr{P}(X) \rightarrow\{0,1\}$ (where $\mathscr{P}(X)$ is the power set of $X$ ) which is finitely additive and satisfies $\mu(X)=1$. By a measure we mean a measure on some set.

Later in the paper we shall have to deal with ultrapowers with respect to two kinds of towers of measures: extenders and sequences of measures associated with homogeneous trees. A bit of the theory of these two kinds of ultrapowers can be developed in a common framework, and that will be our first task. Our framework will be just general enough to cover our two examples.

Let $D$ be a directed nonempty set of sets: if $a, b \in D$ then there is a $c \in D$ such that $a \cup b \subseteq c$. Suppose that $Z$ is a set and $\left\langle\mu_{a} \mid a \in D\right\rangle$ is such that

(1) each $\mu_{a}$ is a countably additive measure on ${ }^{a} Z=\{f \mid f: a \rightarrow Z\}$; 
(2) the $\mu_{a}$ are compatible: if $a \subseteq b$ and $\mu_{a}(X)=1$, then

$$
\mu_{b}(\{f|f| a \in X\})=1 .
$$

We wish to define the ultrapower (of the universe $V$ ) by $\left\langle\mu_{a} \mid a \in D\right\rangle$. (This will really be a direct limit of ultrapowers rather than an ultrapower proper, but calling it an "ultrapower" is by now standard.) Suppose $F:{ }^{a} Z \rightarrow V$ and $G:{ }^{b} Z \rightarrow V$ with $a, b \in D$. We say

$$
F \sim G \Leftrightarrow \mu_{c}(\{h \mid F(h \mid a)=G(h \mid b)\})=1
$$

for some $c \supseteq a \cup b$ with $c \in D$ (iff, by directedness and compatibility, $\mu_{c}(\{h \mid$ $F(h\lceil a)=G(h\lceil b)\})=1$ for all $c \supseteq a \cup b$ with $c \in D$. Let $\llbracket F \rrbracket$ be the set of all members of minimal rank of the equivalence class of $F$ with respect to the equivalence relation $\sim$. For $F:{ }^{a} Z \rightarrow V$ and $G:{ }^{b} Z \rightarrow V$, let $\llbracket F \rrbracket E \llbracket G \rrbracket \Leftrightarrow$ $\mu_{c}(\{h \mid F(h \mid a) \in G(h\lceil b)\})=1$ for some (all) $c \supseteq a \cup b$. The ultrapower by $\left\langle\mu_{a} \mid a \in D\right\rangle$, Ult $\left(V ;\left\langle\mu_{a} \mid a \in D\right\rangle\right)$ is the proper class model for the language of set theory whose domain is the class of $\llbracket F \rrbracket$ such that $(\exists a \in D)\left(F:{ }^{a} Z \rightarrow V\right)$, with " $\in$ " interpreted by $E$. (The only models we shall deal with in this paper are models for the language of set theory, sometimes with constants added.)

Lośs Theorem holds for our ultrapower construction: For any formula $\varphi\left(v_{1}, \ldots, v_{n}\right)$ and any $F_{1}, \ldots, F_{n}, \varphi\left[\llbracket F_{1} \rrbracket, \ldots, \llbracket F_{n} \rrbracket\right]$ holds in $\operatorname{Ult}\left(V ;\left\langle\mu_{a}\right|\right.$ $a \in D\rangle)$ if and only if $\mu_{c}\left(\left\{h \mid \varphi\left[F_{1}\left(h \mid a_{1}\right), \ldots, F_{n}\left(h \mid a_{n}\right)\right]\right.\right.$ holds in $\left.\left.V\right\}\right)=1$, for some (all) $c \supseteq \cup_{1 \leq j \leq n} a_{j}$, where ${ }^{a_{j}} Z=\operatorname{domain}\left(F_{j}\right)$. Thus we get an elementary embedding $i=i_{\left\langle\mu_{a} \mid a \in D\right\rangle}$ with $i: V \rightarrow \operatorname{Ult}\left(V ;\left\langle\mu_{a} \mid a \in D\right\rangle\right)$. (Note our systematic abuse of notation: we do not distinguish between a model and its domain unless there is a possibility of confusion. Thus we write $V$ above, meaning the proper class model $(V ; \in)$, and we often write $\operatorname{Ult}\left(V ;\left\langle\mu_{a} \mid a \in D\right\rangle\right)$ or the like when we are talking of the domain of this model.) $i$ is defined by $i(x)=\llbracket c_{x}^{a} \rrbracket$ for some (all) $a \in D$, where $c_{x}^{a}(f)=x$.

$\operatorname{Ult}\left(V ;\left\langle\mu_{a} \mid a \in D\right\rangle\right)$ may or may not be wellfounded. If it is wellfounded, then it is isomorphic to a unique transitive class by a unique isomorphism.

Convention. If $\operatorname{Ult}\left(V ;\left\langle\mu_{a} \mid a \in D\right\rangle\right)$ is wellfounded, we let $\llbracket F \rrbracket$ be, not the equivalence class of $F$, but rather its image under the isomorphism. (It is usual to use "Ult" in this way; what is unusual is our use of "Ult" for the literal ultrapower in the nonwellfounded case.) This convention applies also to ordinary ultrapowers by single measures. (These are always wellfounded for countably additive measures.)

There is an alternative way of building the ultrapower by $\left\langle\mu_{a} \mid a \in D\right\rangle$. For each $a \in D$ we can form the ordinary ultrapower $\operatorname{Ult}\left(V ; \mu_{a}\right)$. Since $\mu_{a}$ is countably additive, $\operatorname{Ult}\left(V ; \mu_{a}\right)$ is by our convention a transitive class. We have the elementary embedding $i_{\mu_{a}}: V \rightarrow \operatorname{Ult}\left(V ; \mu_{a}\right)$ given by $i_{\mu_{a}}(x)=\llbracket c_{x}^{a} \rrbracket_{\mu_{a}}$. (We use the subscript $\mu_{a}$ to indicate in what ultrapower $c_{x}^{a}$ is representing the object.) For $a, b \in D$ with $a \subseteq b$, let

$$
j_{a, b}: \operatorname{Ult}\left(V ; \mu_{a}\right) \rightarrow \operatorname{Ult}\left(V ; \mu_{b}\right)
$$


be given by $j_{a, b}\left(\llbracket F \rrbracket_{\mu_{a}}\right)=\llbracket F^{\prime} \rrbracket_{\mu_{b}}$, where $F^{\prime}(g)=F(g \mid a)$. By compatibility, the functions $j_{a, b}$ are well-defined and are elementary embeddings.

$$
\left(\left\langle\operatorname{Ult}\left(V, \mu_{a}\right) \mid a \in D\right\rangle,\left\langle j_{a, b} \mid a \subseteq b \& a \in D \& b \in D\right\rangle\right)
$$

is a directed system of models and elementary embeddings. Let $\left(\left\langle M,<j_{a, \infty}\right|\right.$ $a \in D\rangle)$ be the direct limit of this system. $M$ is canonically isomorphic to $\operatorname{Ult}\left(V ;\left\langle\mu_{a} \mid a \in D\right\rangle\right)$. We shall not distinguish notationally between these two objects. Note that if the empty set $\varnothing \in D$, then $i_{\left\langle\mu_{a} \mid a \in D\right\rangle}$ corresponds to $j_{\varnothing, \infty}$.

Lemma 1.1. The following are equivalent.

(a) The ultrapower by $\left\langle\mu_{a} \mid a \in D\right\rangle$ is not wellfounded.

(b) There are a countable $D^{\prime} \subseteq D$ and $\left\langle X_{a} \mid a \in D^{\prime}\right\rangle$ with $\mu_{a}\left(X_{a}\right)=1$ for each $a \in D^{\prime}$ such that there is no $f: \cup D^{\prime} \rightarrow Z$ with $f \mid a \in X_{a}$ for all $a \in D^{\prime}$.

Moreover, if $D$ is countable then (a) and (b) are equivalent to:

(c) There is $\left\langle X_{a} \mid a \in D\right\rangle$ with each $\mu_{a}\left(X_{a}\right)=1$ such that there is no $f: \bigcup D \rightarrow Z$ with $(\forall a \in D) f \uparrow a \in X_{a}$.

Proof. We first show (a) $\Rightarrow(\mathrm{b})$. Let $\left\langle\llbracket F_{i} \rrbracket \mid i \in \omega\right\rangle$ be an infinite descending sequence of "ordinals" of $\operatorname{Ult}\left(V ;\left\langle\mu_{a} \mid a \in D\right\rangle\right)$. For each $i \in \omega$, let $F_{i}:{ }^{a_{i}} Z \rightarrow \mathrm{ON}$ with $a_{i} \in D$. By directedness and compatibility, we may assume that $i \leq j \Rightarrow a_{i} \subseteq a_{j}$. Let $D^{\prime}=\left\{a_{i} \mid i \in \omega\right\}$. Let $X_{a_{0}}={ }^{a_{0}} Z$ and let $X_{a_{i+1}}=\left\{f \in{ }^{a_{i+1}} Z \mid F_{i+1}(f)<F_{i}\left(f \mid a_{i}\right)\right\}$. Clearly $\mu_{a_{i}}\left(X_{a_{i}}\right)=1$ for each $i$. Suppose $f: \bigcup_{i \in \omega} a_{i} \rightarrow Z$ and $f \uparrow a_{i} \in X_{a_{i}}$ for all $i \in \omega$. Then $F_{i+1}\left(f \uparrow a_{i+1}\right)<F_{i}\left(f \mid a_{i}\right)$ for each $i$, giving us an infinite descending sequence of ordinals in $V$ and so a contradiction.

Now let us show (b) $\Rightarrow(\mathrm{a})$. Let $D^{\prime}$ and $\left\langle X_{a} \mid a \in D^{\prime}\right\rangle$ witness (b). Let $D^{\prime}=\left\{a_{i} \mid i \in \omega\right\}$. By directedness, compatibility, and countable additivity, we may assume that $i \leq j \Rightarrow a_{i} \subseteq a_{j}$ and that whenever $i \leq j \& f \in X_{a_{j}}$ then $f \uparrow a_{i} \in X_{a_{i}}$. Let

$$
\begin{array}{r}
T=\left\{\left\langle f_{i} \mid i<n\right\rangle \mid n \in \omega \&(\exists f)\left(f: a_{n-1} \rightarrow Z \&(\forall i<n) f_{i}=f \mid a_{i}\right)\right. \\
\left.\&(\forall i<n) f_{i} \in X_{a_{i}}\right\} .
\end{array}
$$

If we partially order $T$ by $s_{1} \prec s_{2} \Leftrightarrow s_{1}$ properly extends $s_{2}$, then $(T, \prec)$ is a wellfounded partial ordering. Let

$$
F_{n}(f)=\operatorname{rank}_{T}\left\{\left\langle f_{i} \mid i<n+1\right\rangle\left|(\forall i<n+1)\left(f_{i}=f \mid a_{i}\right)\right\rangle\right\}
$$

for $f \in X_{a_{n}}$, where $\operatorname{rank}_{T}(s)$ is defined inductively for $s \in T$ by

$$
\operatorname{rank}_{T}(s)=\sup \left\{\operatorname{rank}_{T}\left(s^{\prime}\right)+1 \mid s^{\prime} \text { properly extends } s \& s^{\prime} \in T\right\}
$$

The $\llbracket F_{n} \rrbracket$ form an infinite descending sequence of ordinals of $\operatorname{Ult}\left(V ;\left\langle\mu_{a}\right|\right.$ $a \in D\rangle)$. 
(b) $\Rightarrow$ (c) in general. To see this let $D^{\prime}$ and $\left\langle X_{a} \mid a \in D^{\prime}\right\rangle$ witness $B$. For $a \in D^{\prime}-D$ let $X_{a}={ }^{a} Z$. $\left\langle X_{a} \mid a \in D\right\rangle$ witnesses (c). If $D$ is countable, obviously (c) $\Rightarrow$ (b).

Iterations. We need to consider not just ultrapowers and their elementary embeddings but also iterated ultrapowers and the associated iterations of embeddings. First we prove two simple very general facts.

Lemma 1.2. Let $M_{k}, k \in \omega$, be transitive proper class models of ZFC and let $\left\langle i_{m, n} \mid m \leq n \in \omega\right\rangle$ be such that each $i_{m, n}: M_{m} \rightarrow M_{n}$ is an elementary embedding and such that $i_{n, p} \circ i_{m, n}=i_{m, p}$ for $m \leq n \leq p$, where $\circ$ denotes composition. Let $\left(M_{\infty},\left\langle i_{k, \infty} \mid k \in \omega\right\rangle\right)$ be the direct limit of the system $\left(\left\langle M_{k} \mid k \in \omega\right\rangle,\left\langle i_{m, n} \mid m \leq n \in \omega\right\rangle\right)$.

(a) $M_{\infty}$ is illfounded if and only if there are ordinals $\beta_{k}, k \in \omega$, with each $\beta_{k} \in M_{k}$ and $\left(m<n \Rightarrow i_{m, n}\left(\beta_{m}\right)>\beta_{n}\right)$.

(b) Assume $M_{0}=V$. Let $e_{k} \in M_{k}$ for each $k \in \omega$. Define measures $\nu_{k}$ as follows:

$$
\nu_{k}(X)=1 \Leftrightarrow\left\langle i_{m, k}\left(e_{m}\right) \mid m<k\right\rangle \in i_{o, k}(X) .
$$

The $\nu_{k}$ are compatible and they are $\kappa$-complete, where $\kappa$ is the smallest critical point of the $i_{m, n}$. Moreover, $\operatorname{Ult}\left(V ;\left\langle\nu_{k} \mid k \in \omega\right\rangle\right)$ is wellfounded if $M_{\infty}$ is wellfounded.

Proof. (a) If the $\beta_{k}$ 's exist, then $\left\langle i_{m, \infty}\left(\beta_{m}\right) \mid m \in \omega\right\rangle$ is an infinite descending sequence of ordinals of $M_{\infty}$. Suppose then that $M_{\infty}$ is illfounded, and let us show that the $\beta_{k}$ 's exist. Let $\left\langle b_{k} \mid k \in \omega\right\rangle$ be an infinite descending sequence of ordinals of $M_{\infty}$. Let $\gamma_{k}$ and $m_{k}$ be such that $i_{m_{k}, \infty}\left(\gamma_{k}\right)=b_{k}$. We may assume without loss of generality that $m_{0}=0$ and $k_{1}<k_{2} \Rightarrow m_{k_{1}}<m_{k_{2}}$. For $0 \leq q<m_{k+1}-m_{k}$, let $\beta_{m_{k}+q}=i_{m_{k}, m_{k}+q}\left(\omega \cdot \gamma_{k}+m_{k+1}-m_{k}-q\right)$.

(b) The compatibility of the $\nu_{k}$ is easily verified, as is the $\kappa$-completeness of the $\nu_{k}$. For $m \leq n \in \omega$, let $j_{m, n}: \operatorname{Ult}\left(V ; \nu_{m}\right) \rightarrow \operatorname{Ult}\left(V ; \nu_{n}\right)$ be the elementary embedding as defined earlier (in the alternative definition of Ult $\left(V ;\left\langle\mu_{a}\right|\right.$ $a \in D\rangle))$. For each $k \in \omega$, define $\pi_{k}: \operatorname{Ult}\left(V ; \nu_{k}\right) \rightarrow M_{k}$ by

$$
\pi_{k}\left(\llbracket F \prod_{\nu_{k}}\right)=\left(i_{0, k}(F)\right)\left(\left\langle i_{m, k}\left(e_{m}\right) \mid m<k\right\rangle\right) .
$$

It is easy to see that $\pi_{k}$ is well defined and is an elementary embedding. Moreover, we have the following commutative diagram:

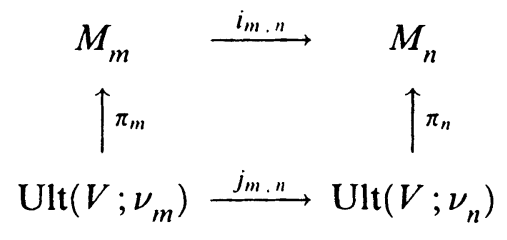


This is because

$$
\begin{aligned}
\pi_{n}\left(j_{m, n}\left(\llbracket F \rrbracket_{\nu_{m}}\right)\right) & =\pi_{n}\left(\llbracket F^{\prime} \prod_{\nu_{n}}\right) \\
& =\left(i_{0, n}\left(F^{\prime}\right)\right)\left(\left\langle i_{m^{\prime}, n}\left(e_{m^{\prime}}\right) \mid m^{\prime}<n\right\rangle\right) \\
& =\left(i_{0, n}(F)\right)\left(\left\langle i_{m^{\prime}, n}\left(e_{m^{\prime}}\right) \mid m^{\prime}<m\right\rangle\right) \\
& =i_{m, n}\left(\left(i_{0, m}(F)\right)\left(\left\langle i_{m^{\prime}, m}\left(e_{m^{\prime}}\right) \mid m^{\prime}<m\right\rangle\right)\right) \\
& =i_{m, n}\left(\pi_{m}\left(\llbracket F \prod_{\nu_{m}}\right)\right),
\end{aligned}
$$

where $F^{\prime}(f)=F(f \uparrow m)$. Hence we get an elementary embedding $\pi_{\infty}$ : $\operatorname{Ult}\left(V ;\left\langle\nu_{k} \mid k \in \omega\right\rangle\right) \rightarrow M_{\infty}$ defined by $\pi_{\infty}\left(j_{k, \infty}(x)\right)=i_{k, \infty}\left(\pi_{k}(x)\right)$. If $M_{\infty}$ is wellfounded, then $\left.\operatorname{Ult}\left(V ;\left\langle\nu_{k}\right| k \in \omega\right)\right)$, which is embedded into $M_{\infty}$, must be wellfounded also.

As in the case of a single measure, the notion $\operatorname{Ult}\left(M,\left\langle\mu_{a} \mid a \in D\right\rangle\right)$ makes sense when $M$ is a transitive (set or class) model of ZFC and $M$ satisfies " $D$ is a directed family of sets and $\left\langle\mu_{a} \mid a \in D\right\rangle$ have, with some $Z$, properties (1) and (2)." ((1) and (2) are the properties formulated at the beginning of this section.) Just let $\operatorname{Ult}\left(M ;\left\langle\mu_{a} \mid a \in D\right\rangle\right)$ be $\left(\operatorname{Ult}\left(V ;\left\langle\mu_{a} \mid a \in D\right\rangle\right)\right)^{M}$.

By an internal iteration on $M$ of length $q+1$, with $M$ a transitive proper class model of ZFC and $q \in \omega$, let us mean a system $\left(\left\langle M_{k} \mid k \leq q\right\rangle,\left\langle i_{m, n}\right|\right.$ $m \leq n \leq q\rangle$ ) such that each $M_{k}$ is a transitive proper class model of ZFC with $M_{0}=M$, each $i_{m, n}: M_{m} \rightarrow M_{n}$ is an elementary embedding, $i_{n, p} \circ i_{m, n}=i_{m, p}$ whenever $m \leq n \leq p$, each $i_{k, k+1}$ is $i_{\left\langle\mu_{a}^{k} \mid a \in D_{k}\right\rangle}$ for some $Z_{k}$ and $\left\langle\mu_{a} \mid a \in D_{k}\right\rangle$ with $M_{k}$ satisfying " $D_{k}$ is a directed family of sets and (1) and (2) hold of $Z_{k}$ and $\left\langle\mu_{a}^{k} \mid a \in D_{k}\right\rangle$ ", and each $M_{k+1}=\operatorname{Uit}\left(M_{k} ;\left\langle\mu_{a}^{b} \mid a \in D_{k}\right\rangle\right)$.

An internal iteration on $M$ of length $\omega$ is a system $\left(\left\langle M_{k} \mid k \in \omega\right\rangle,\left\langle i_{m, n}\right|\right.$ $m \leq n \in \omega\rangle)$ such that each $\left(\left\langle M_{k} \mid k \leq q\right\rangle,\left\langle i_{m, n} \mid m \leq n \leq q\right\rangle\right)$ is an internal iteration on $M$ of length $q+1$.

Remark. The foregoing definitions are an example of our unnecessary use of proper classes. The models $M_{k}$ and the embeddings $i_{m, n}$ are completely determined by the systems of measures and the model $M$. Thus we could have defined an internal iteration as a sequence $\left\langle\left\langle\mu_{a}^{k} \mid a \in D_{k}\right\rangle \mid k \in \omega\right\rangle$. This would make an internal iteration a set instead of a proper class.

Lemma 1.3. If $\left(\left\langle M_{k} \mid k \in \omega\right\rangle,\left\langle i_{m, n} \mid m \leq n \in \omega\right\rangle\right)$ is an internal iteration on $M$ of length $\omega$ and $\left(M_{\infty},\left\langle i_{m, \infty} \mid m \in \omega\right\rangle\right)$ is the direct limit of this internal iteration, then $M_{\infty}$ is wellfounded.

Proof. Assume that the lemma is false. Let $M$ be such that there is an internal iteration on $M$ of length $\omega$ witnessing the falsity of the lemma. There is then, by Lemma 1.2(a), an ordinal $\alpha$ such that there is an internal iteration $\left(\left\langle M_{k} \mid k \in \omega\right\rangle,\left\langle i_{m, n} \mid m \leq n \in \omega\right\rangle\right)$ on $M$ of length $\omega$ and there is a sequence $\left\langle\beta_{k} \mid k \in \omega\right\rangle$ such that each $\beta_{k}$ is an ordinal and $i_{m, n}\left(\beta_{m}\right)>\beta_{n}$ whenever $m<n$ and such that $\beta_{0}=\alpha$. Let $\alpha$ be the least such ordinal and 
let $\left\langle M_{k} \mid k \in \omega\right\rangle,\left\langle i_{m, n} \mid m \leq n \in \omega\right\rangle$, and $\left\langle\beta_{k} \mid k \in \omega\right\rangle$ witness that $\alpha$ has this property. Let $\left\langle\left\langle\mu_{a}^{k} \mid a \in D_{k}\right\rangle \mid k \in \omega\right\rangle$ witness that this is an internal iteration. Let $\gamma$ be an ordinal such that both $\left\langle\beta_{k} \mid k \in \omega\right\rangle$ and $\left\langle\left\langle\mu_{a}^{k} \mid a \in D_{k}\right\rangle \mid k \in \omega\right\rangle$ belong to $V_{\gamma}$.

Consider a formula $\varphi\left(v_{1}, v_{2}\right)$, indicated as follows.

$\varphi\left(v_{1}, v_{2}\right): \quad v_{1}$ and $v_{2}$ are ordinals and there are $\left\langle\left\langle\hat{\mu}_{a}^{k} \mid a \in \widehat{D}_{k}\right\rangle \mid k \in \omega\right\rangle$ and $\left\langle\hat{\beta}_{k} \mid k \in \omega\right\rangle$, both in $V_{v_{2}}$, and there is an internal iteration $\left(\left\langle\widehat{M}_{k}\right| k \in\right.$ $\left.\omega\rangle,\left\langle\hat{i}_{m, n} \mid m \leq n \in \omega\right\rangle\right)$ on $V$ of length $\omega$ such that $\left\langle\left\langle\hat{\mu}_{a}^{k} \mid a \in \widehat{D}_{k}\right\rangle \mid k \in \omega\right\rangle$ and $\left\langle\hat{\beta}_{k} \mid k \in \omega\right\rangle$ witness that this iteration is internal, such that $\hat{i}_{m, n}\left(\hat{\beta}_{m}\right)>\hat{\beta}_{n}$ whenever $m<n$, and such that $\hat{\beta}_{0}=v_{1}$.

$M$ satisfies $\varphi[\alpha, \gamma]$, for otherwise the set of all finite attempts to build a witness that $M$ satisfies $\varphi[\alpha, \gamma]$, partially ordered by putting proper extensions before the initial parts they extend, is wellfounded in $M$. By the absoluteness of wellfoundedness, this partial ordering is really wellfounded. But $\left\langle\beta_{k} \mid k \in \omega\right\rangle$ and $\left\langle\left\langle\mu_{a}^{k} \mid a \in D_{k}\right\rangle \mid k \in \omega\right\rangle$ give an infinite descending chain in this partial ordering.

Similarly $M$ satisfies " $\alpha$ is the least ordinal $\xi$ such that $\varphi(\xi, \gamma)$." Hence $M_{1}$ satisfies " $i_{0,1}(\alpha)$ is the least ordinal $\xi$ such that $\varphi\left(\xi, i_{0,1}(\gamma)\right)$." But $\left(\left\langle M_{k+1} \mid k \in \omega\right\rangle,\left\langle i_{m+1, n+1} \mid m \leq n \in \omega\right\rangle\right)$ is an internal iteration on $M_{1}$. Hence absoluteness arguments as before show that $M_{1}$ satisfies $\varphi\left[\beta_{1}, \gamma\right]$. Since $\gamma \leq i_{0,1}(\gamma), M_{1}$ satisfies $\varphi\left[\beta_{1}, i_{0,1}(\gamma)\right]$. Since $\beta_{1}<i_{0,1}(\alpha)$, this is a contradiction.

Extenders, to which we now turn, are a refinement due to A. Dodd and R. Jensen of an analysis by W. Mitchell of arbitrary elementary embeddings $j: V \rightarrow M$ in terms of directed systems of measures. Our definition here is a little more general than that of Dodd and Jensen (see [1]), since it is convenient for us to permit arbitrary transitive sets and not just ordinal numbers as supports of our extenders.

We shall in fact give two different, but essentially equivalent, definitions of "extender". The first definition is more elegant and fits into the general theory we have been developing. The second, which we shall use in the bulk of the paper, has special technical advantages for the uses to which we shall put extenders.

Let $Y$ be a transitive set and let $\kappa$ be a cardinal number. An extender with critical point $\kappa$ and support $Y$ is a system $E=\left\langle E_{a} \mid a \in{ }^{<\omega}[Y]\right\rangle \quad\left({ }^{<\omega}[Y]\right.$ is the collection of all finite subsets of $Y$ ) with the following properties:

(i) Each $E_{a}$ is a $\kappa$-complete measure on ${ }^{a}\left(V_{\kappa}\right)$, and at least one $E_{a}$ is not $\kappa^{+}$-complete.

(ii) The $E_{a}$ are compatible in the sense defined at the beginning of this section.

(iii) $E_{a}(\{f \mid f:(a, \in) \simeq(\operatorname{range}(f) ; \in)\})=1$. 
(iv) If $F:{ }^{a}\left(V_{\kappa}\right) \rightarrow V_{\kappa}$ and $E_{a}(\{f \mid F(f) \in U(\operatorname{range}(f))\})=1$, then there is a $y \in Y$ such that $E_{a \cup\{y\}}(\{f \mid F(f \mid a)=f(y)\})=1$.

(v) $\operatorname{Ult}\left(V ;\left\langle E_{a} \mid a \in^{<\omega}[Y]\right\rangle\right)$ is wellfounded.

Remarks. (a) Properties (i) and (ii) imply that $V_{\kappa}$ and $\left\langle E_{a} \mid a \in{ }^{<\omega}[Y]\right\rangle$ satisfy (1) and (2) above (with $Z=V_{\kappa}$ and $D={ }^{<\omega}[Y]$.

(b) One could also speak of $\kappa$ as the completeness of $E$ instead of the critical point of $E$.

(c) The term "extender" should really also be applied if we replace $V_{\kappa}$ by an arbitrary set. We use the more restrictive definition here because it is convenient to deal only with extenders in our restrictive sense.

Lemma 1.4. Let $E=\left\langle E_{a} \mid a \in{ }^{<\omega}[Y]\right\rangle$ be an extender with critical point $\kappa$. Let $i_{E}: V \rightarrow \operatorname{Ult}(V ; E)$ be the canonical elementary embedding. Then $i_{E} \uparrow V_{\kappa}$ is the identity but $i_{E}(\kappa)>\kappa$. (Thus $\kappa$ is the critical point, in the usual sense, of $\left.i_{E}.\right)$

Proof. The proof of the first assertion is just like that for the corresponding fact about the embedding from a single $\kappa$-complete measure on $\kappa$ : One proves, by induction on rank, that constant functions with values in $V_{\kappa}$ represent their values, using the $\kappa$-completeness of the $E_{a}$. For the second assertion, let $E_{a}$ be not $\kappa^{+}$-complete. Let $X_{\alpha}$, for $\alpha<\kappa$, be such that $E_{a}\left(X_{\alpha}\right)=1$ but suppose also that $E_{a}\left(\bigcap_{\kappa<\kappa} X_{\alpha}\right)=0$. Let $F:{ }^{a}\left(V_{\kappa}\right) \rightarrow \kappa$ be given by $F(f)=$ the least $\alpha<\kappa$ such that $f \notin X_{\alpha}$. It is easy to see that $\alpha<\llbracket F \rrbracket$ for each $\alpha<\kappa$ but $\llbracket F \rrbracket<i_{E}(\kappa)$.

Lemma 1.5. Let $E=\left\langle E_{a} \mid a \in{ }^{<\omega}[Y]\right\rangle$ be an extender with $\operatorname{crit}(E)$ (the critical point of $E)=\kappa$. For each $y \in Y$ and each $a \in^{<\omega}[Y]$ with $y \in a$, let

$$
H_{y}^{a}(f)=f(y)
$$

for all $f \in{ }^{a}\left(V_{\kappa}\right)$. In Ult $(V ; E), \quad y=\llbracket H_{y}^{a} \rrbracket$.

Proof. We prove the lemma for all $y$ and $a$ by induction on $\operatorname{rank}(y)$. Note first that the definition of the ultrapower gives directly that $\llbracket H_{y}^{a} \rrbracket=\llbracket H_{y}^{b} \rrbracket$ for any $a$ and $b$ with $y \in a \cap b$.

Suppose the lemma holds for all $z \in Y$ with $\operatorname{rank}(z)<\operatorname{rank}(y)$. Let $z \in y$. Let $a$ be such that $\{z, y\} \subseteq a$. By clause (iii) in the definition of extender, $E_{a}(\{f \mid f(z) \in f(y)\})=1$. Thus $E_{a}\left(\left\{f \mid H_{z}^{a}(f) \in H_{y}^{a}(f)\right\}\right)=1$, and so $\llbracket H_{z}^{a} \rrbracket \in \llbracket H_{y}^{a} \rrbracket$. Since $z=\llbracket H_{z}^{a} \rrbracket$ by our inductive assumption, it follows that $z \in \llbracket H_{y}^{a} \rrbracket$. By the remark at the beginning of the proof, $z \in \llbracket H_{y}^{b} \rrbracket$ for any $b$ with $y \in b$.

Now suppose $y \in a$ and $\llbracket F \rrbracket \in \llbracket H_{y}^{a} \rrbracket$ with $F:{ }^{a}\left(V_{\kappa}\right) \rightarrow V$. Then $E_{a}(\{f \mid F(f) \in f(y)\})=1$ so $E_{a}(\{f \mid F(f) \in \bigcup(\operatorname{range}(f))\})=1$. By clause (iv) there is a $z \in Y$ such that $E_{a \cup\{z\}}(\{f \mid F(f \mid a)=f(z)\})=1$. Hence $\llbracket F \rrbracket=\llbracket H_{z}^{a \cup\{z\}} \rrbracket=z$. But we also have $E_{a \cup\{z\}}(\{f \mid f(z) \in f(y)\})=1$, and so (iii) gives that $z \in y$. Hence $\llbracket F \rrbracket \in y$ as required. 
Example 1. Let $j: V \rightarrow N$ be an elementary embedding with $N$ transitive. Let $\kappa \in Y \subseteq V_{j(\kappa)} \cap N$ with $Y$ transitive, where $\kappa=\operatorname{crit}(j)$. For each $a \in{ }^{<\omega}[Y]$ define a measure $E_{a}$ on ${ }^{a}\left(V_{\kappa}\right)$ by

$$
E_{a}(X)=1 \Leftrightarrow j^{-1}\lceil j(a) \in j(X) .
$$

Let $E=\left\langle E_{a} \mid a \in^{<\omega}[Y]\right\rangle$.

It is easy to see that each $E_{a}$ is a $\kappa$-complete measure on ${ }^{a}\left(V_{\kappa}\right)$ and that the $E_{a}$ are compatible. $E_{\{\kappa\}}$ is not $\kappa^{+}$-complete, since $j \operatorname{moves} \kappa=\operatorname{crit}(j)$. Thus (i) and (ii) in the definition of extender hold. (iii) holds since $j^{-1} \uparrow j(a)$ : $(j(a), \in) \simeq(a, \in)$. To verify (iv), let $F:{ }^{a}\left(V_{\kappa}\right) \rightarrow V$ with

$$
E_{a}(\{F \mid F(f) \in \bigcup(\operatorname{range}(f))\})=1
$$

By definition, $(j(F))\left(j^{-1} \uparrow j(a)\right) \in \bigcup(a)$. Thus $(j(F))\left(j^{-1} \uparrow j(a)\right) \in y$ for some $y \in a$. Therefore $(j(F))\left(j^{-1} \mid j(a)\right)=z$ for some $z \in Y$, since $Y$ is transitive. By the definition of $E_{a \cup\{z\}}$,

$$
E_{a \cup\{z\}}(\{f \mid F(f \mid a)=f(z)\})=1 .
$$

Let $k: \operatorname{Ult}(V ; E) \rightarrow N$ be defined by

$$
k(\llbracket F \rrbracket)=(j(F))\left(j^{-1} \uparrow j(a)\right)
$$

for $F \in{ }^{a}\left(V_{\kappa}\right)$. It is easy to check that $k$ is well-defined and is an elementary embedding. Since, in particular, this means that $k$ is a monomorphism of $\operatorname{Ult}(V ; E)$ into the wellfounded model $N$, we have $(\mathrm{v})$ and so have that $E$ is an extender.

In addition, we have the following commutative diagram:

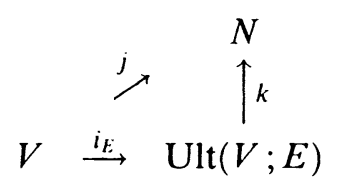

To see this, note that if $c_{y}^{a}:{ }^{a}\left(V_{\kappa}\right) \rightarrow\{y\}$ is the constant function then $k\left(i_{E}(y)\right)=k\left(\llbracket c_{y}^{a} \rrbracket\right)=\left(j\left(c_{y}^{a}\right)\right)\left(j^{-1}\lceil j(a))=j(y)\right.$.

Suppose $y \in Y$. By Lemma 1.5, $k(y)=k\left(\llbracket H_{y}^{a} \rrbracket\right)=\left(j\left(H_{y}^{a}\right)\right)\left(j^{-1} \uparrow j(a)\right)=$ $\left(j^{-1} \uparrow j(a)\right)(j(y))=j^{-1}(j(y))=y$. In other words, $k \uparrow Y$ is the identity.

We shall call the extender $E$ defined from $j$ and $Y$ as above the extender derived from $j$ with support $Y$.

If $E$ is an extender with support $Y$, then Lemma 1.5 implies that $Y \subseteq$ $\operatorname{Ult}(V ; E)$, and so we can define the extender $E^{\prime}$ derived from $i_{E}$ with support $Y$. We see as follows that $E^{\prime}$ is just $E$ itself: Let $E=\left\langle E_{a} \mid a \in^{<\omega}[Y]\right\rangle$ and let $E^{\prime}=\left\langle E_{a}^{\prime} \mid a \epsilon^{<\omega}[Y]\right\rangle$. We have that $E_{a}^{\prime}(X)=1 \Leftrightarrow i_{E}^{-1} \mid i_{E}(a) \in i_{E}(X)$. 
For $y \in a, i_{E}^{-1}\left(i_{E}(y)\right)=y=\llbracket H_{y}^{a} \rrbracket$. Hence $i_{E}^{-1} \mid i_{E}(a)=\llbracket\langle\langle f(y) \mid y \in a\rangle|$ $\left.f \in{ }^{a}\left(V_{\kappa}\right)\right\rangle \rrbracket=\llbracket\left\langle f \mid f \in{ }^{a}\left(V_{\kappa}\right)\right\rangle \rrbracket=\llbracket \operatorname{id}_{a_{(}\left(V_{\kappa}\right)} \rrbracket$. Hence $E_{a}^{\prime}(X)=1 \Leftrightarrow \llbracket \operatorname{id}_{a_{(}\left(V_{\kappa}\right)} \rrbracket \in$ $i_{E}(X) \Leftrightarrow E_{a}(\{f \mid f \in X\})=1 \Leftrightarrow E_{a}(X)=1$.

Thus every extender is the extender derived from some $j: V \rightarrow N$ with support some $Y \subseteq V_{j(\kappa)} \cap N$. We could, if we wished, have made this our definition of extender and have taken our present definition as a derived result. In [10] we do define extenders in this way.

Example 2. Let $E$ be an extender with $\operatorname{support}(E)=Y$, i.e. with $Y$ the support of $E$. Let $Y^{\prime} \subseteq Y$ be transitive with $Y^{\prime} \nsubseteq V_{\kappa}$. Let $E^{\prime}=E \backslash Y^{\prime}$, i.e. let $E^{\prime}=\left\langle E_{a} \mid a \in{ }^{<\omega}\left[Y^{\prime}\right]\right\rangle$. It is easy to check that $E^{\prime}$ is an extender with the same critical point as $E$ and with $\operatorname{support}\left(E^{\prime}\right)=Y^{\prime}$. In fact, $E^{\prime}$ is the extender derived from $i_{E}$ with support $Y^{\prime}$, as the argument of two paragraphs ago essentially shows. Thus we have the elementary embedding $k: \operatorname{Ult}\left(V ; E^{\prime}\right) \rightarrow$ $\operatorname{Ult}(V ; E)$ as in Example 1. $k$ can also be defined by $k\left(\llbracket F \rrbracket_{E^{\prime}}\right)=\llbracket F \rrbracket_{E}$, since if $F:{ }^{a}\left(V_{\kappa}\right) \rightarrow V$ then $\left(i_{E}(F)\right)\left(i_{E}^{-1}\left\lceil i_{E}(a)\right)=\left(i_{E}(F)\right)\left(\llbracket \operatorname{id}_{a_{(}\left(V_{\kappa}\right)} \rrbracket_{E}\right)=\llbracket F \rrbracket_{E}\right.$.

We gave the particular definition of extender that we did because it fits directly into the general scheme introduced at the beginning of this section. But our definition has one defect: The measures $E_{a}$ are essentially measures on ${ }^{|a|}\left(V_{\kappa}\right)$ but they are not literally measures on ${ }^{|a|}\left(V_{\kappa}\right)$. This small difference would produce-if we continued to operate with our official definitionnumerous small notational problems. For this reason we shall use an alternative definition of extender (whose specific form was suggested to us by M. Foreman).

We replace $\left\langle E_{a} \mid a \in{ }^{<\omega}[Y]\right\rangle$ by $\left\langle\widehat{E}(q) \mid q \in{ }^{<\omega} Y\right\rangle$ where ${ }^{<\omega} Y$ is the collection of all finite sequences of elements of $Y$. Each $\widehat{E}(q)$ is a measure on ${ }^{\text {eh }(q)}\left(V_{\kappa}\right)$, where $\ell h(q)$ is the length of $q$. Let

$$
\widehat{E}(q)(X)=E_{\text {range }(q)}\left(\left\{f \mid g_{f} \in X\right\}\right),
$$

where $g_{f}(n)=f(q(n))$. We no longer have a system satisfying (1) and (2), so the ultrapower must be defined differently: Elements of the ultrapower are of the form $\llbracket F \mathbb{\rrbracket}_{q}$, when $q \in{ }^{<\omega} Y$ and $F:{ }^{\ln (q)}\left(V_{\kappa}\right) \Rightarrow V$. $\llbracket F \mathbb{1}_{q}=\llbracket G \mathbb{1}_{r} \Leftrightarrow$ $\widehat{E}\left(q^{-} r\right)\left(\left\{h \mid F(h \mid \ell h(q))=G(\langle h(\ell h(q)+m|m<\ell h(r)\rangle)\})=1 .\left(q^{-} r\right.\right.\right.$ is the concatenation of the sequences $q$ and $r$.) The characterization of when $\llbracket F \rrbracket_{q} \in \llbracket G \rrbracket_{r}$ is totally analogous. We leave to the reader the easy verification that this ultrapower is isomorphic to the ultrapower by the system $E$ described previously.

Our new definition of extender is thus finally: An extender with critical point $\kappa$ and support $Y$ is a system $\left\langle E(a) \mid a \in{ }^{<\omega} Y\right\rangle$ such that

$\left(\mathrm{i}^{*}\right)$ Each $E(a)$ is a $\kappa$-complete measure on ${ }^{\ell h(a)}\left(V_{\kappa}\right)$ and at least one $E(a)$ is not $\kappa^{+}$-complete.

(ii $\left.{ }^{*}\right)$ The $E(a)$ are compatible in the sense that if $(E(a))(X)=1$ then

$$
\left(E\left(a^{-} b\right)\right)(\{z|z| \ln (a) \in X\})=1 .
$$


$\left(\right.$ iii $\left.^{*}\right) \quad(E(a))(\{z \mid(\forall m<\ell h(a))(\forall n<\ell h(a))(a(m) \in a(n) \Leftrightarrow z(m) \in z(n))\})=$ 1 , and if $\pi$ is a permutation of $\ell h(a)$, then

$$
(E(a \circ \pi))(\{z \circ \pi \mid z \in X\})=(E(a))(X) .
$$

$\left(\right.$ iv $\left.^{*}\right)$ If $F:{ }^{\ell h(a)}\left(V_{\kappa}\right) \rightarrow V_{\kappa}$ and $(E(a))(\{z \mid F(z) \in \bigcup(\operatorname{range}(z))\})=1$, then there is a $y \in Y$ such that

$$
\left(E\left(a^{\Upsilon}\langle y\rangle\right)\right)(\{z \mid F(z \mid \operatorname{lh}(a))=z(\operatorname{lh}(a))\})=1 .
$$

$\left(\mathrm{v}^{*}\right)$ The ultrapower $\operatorname{Ult}(V ; E)$ as described above is wellfounded.

Note that we are reverting to using " $E$ " and " $a$ " when we talk of extenders in the new sense, but we write $E(a)$ instead of $E_{a}$ to indicate that we are dealing with an extender in the new sense. Our two notions of extender are equivalent in that there is a natural one-one correspondence between extenders of the two types, and corresponding extenders have isomorphic ultrapowers.

We shall sometimes want to consider ultrapowers $\operatorname{Ult}(M ; E)$ where $M$ is a set rather than a proper class and where $M$ may not satisfy full ZFC. Let $\mathrm{ZC}$ be $\mathrm{ZFC}$ without replacement (but with Aussonderung). Assume that $M$ is a (set or proper class) model of $\mathrm{ZC}+\Sigma_{2}$-replacement $+(\forall x)(\exists \alpha) x \in V_{\alpha}$. The ultrapower of $M$ by a system $\langle\mu(a) \mid a \in D\rangle$ such that $M$ satisfies " $D$ is a directed set of sets and $\langle\mu(a) \mid a \in D\rangle$ and some $Z$ have properties (1) and (2)" makes sense and is definable in $M$.

We shall often want to form the ultrapower of a model by an extender of a different model. Suppose $M$ and $N$ are transitive (set or class) models of $\mathrm{ZC}+\Sigma_{2}$-replacement $+(\forall x)(\exists \alpha) x \in V_{\alpha}$ and suppose that $V_{\kappa+1} \cap M=V_{\kappa+1} \cap N$ for some ordinal $\kappa$ of $M \cap N$. Suppose $M$ satisfies " $E$ is an extender and $\operatorname{crit}(E)=\kappa$." We can form $\operatorname{Ult}(N ; E)$, the ultrapower of $N$ by $E$, as follows. If $a$ and $b$ belong to ${ }^{<\omega}(\operatorname{support}(E))$ and both $F:{ }^{\ell h(a)}\left(V_{\kappa} \cap N\right) \rightarrow N$ and $G:{ }^{l h(b)}\left(V_{\kappa} \cap N\right) \rightarrow N$ belong to $N$, then

$$
\begin{aligned}
& (F, a) \sim(G, b) \\
& \quad \Leftrightarrow\left(E\left(a^{-} b\right)\right)(\{z \mid F(z\lceil\operatorname{lh}(a))=G(\langle z(\operatorname{lh}(a)+m|m<\operatorname{lh}(b)\rangle)\})=1 .
\end{aligned}
$$

$\sim$ is an equivalence relation, and we can therefore form equivalence classes ( $\cap$ their members' minimal rank) which we denote $\llbracket F \prod_{a}^{N}$. As usual we define Ult $(N ; E)$ and get the embedding $i_{E}^{N}: N \rightarrow \operatorname{Ult}(N ; E)$. (If confusion is possible, we shall henceforth denote the embedding of $M$ itself into $\operatorname{Uit}(M ; E)$ by $i_{E}^{M}$.) $i_{E}^{N}$ is elementary if $N$ satisfies replacement for the domain $V_{\operatorname{crit}(E)}$. In general Ult $(N ; E)$ need not be wellfounded, even if $M$ and $N$ are proper classes. The following lemma gives a useful sufficient condition for its wellfoundedness.

Lemma 1.6. Suppose $M$ and $N$ are transitive (set or class) models of $Z C+\Sigma_{2}$ replacement $+(\forall x)(\exists \alpha) x \in V_{\alpha}$, that $V_{\kappa+1} \cap M=V_{\kappa+1} \cap N$ for some $\kappa \in M \cap N$, 
and that $M$ satisfies " $E$ is an extender and $\operatorname{crit}(E)=\kappa$." Suppose also that $M$ is countably closed, i.e. that ${ }^{\omega} M \subseteq M$. Then $\operatorname{Ult}(N ; E)$ is wellfounded.

Proof. Assume for a contradiction that $\operatorname{Ult}(N ; E)$ is illfounded. Let $\left\langle\llbracket F_{i} \rrbracket_{a_{i}}^{N}\right|$ $i \in \omega\rangle$ be an infinite descending " $\in$-sequence" of Ult $(N ; E)$. We may assume that $i \leq j \Rightarrow a_{i} \subseteq a_{j}$. Just as in the proof of the (a) $\Rightarrow(\mathrm{b})$ part of Lemma 1.1, define $X_{a_{0}}={ }^{\ln \left(a_{0}\right)}\left(V_{\kappa}\right)$ and $X_{a_{i+1}}=\left\{z \in^{\ln \left(a_{i+1}\right)}\left(V_{\kappa}\right) \mid F_{i+1}(z)<F_{i}\left(z \mid \ell h\left(a_{i}\right)\right)\right\}$. Each $X_{a_{i+1}} \in N$ and so each $X_{a_{i+1}} \in M$. As in the proof of Lemma 1.1, there is no $f: \omega \rightarrow V_{\kappa}$ with each $f \mid \ell h\left(a_{i}\right) \in X_{a_{i}}$, or else we have an infinite descending $\in$-sequence in $V$. But $M$ is countably closed, so $\left\langle a_{i} \mid i \in \omega\right\rangle \in M$ and $\left\langle X_{a_{i}} \mid i \in \omega\right\rangle$. But then $M$ does not satisfy " $E$ is an extender," contrary to hypothesis.

Lemma 1.7. Suppose $M$ and $N$ are transitive (set or class) models of $Z C+\Sigma_{2}-$ replacement $+(\forall x)(\exists \alpha) x \in V_{\alpha}$, that $V_{\rho+1} \cap M=V_{\rho+1} \cap N$, that $\kappa \leq \rho \in M \cap N$, that $M$ satisfies " $E$ is an extender with critical point $\kappa$ and support $Y$," and that $\operatorname{Ult}(M ; E)$ and $\operatorname{Ult}(N ; E)$ are wellfounded. Then

(a) $V_{i_{E}^{\prime \prime}(\rho)+1} \cap \operatorname{Ult}(M ; E)=V_{i_{E}^{N}(\rho)+1} \cap \operatorname{Ult}(N ; E)$.

(b) $Y \subseteq \operatorname{Ult}(N ; E)$.

(c) For $x \in V_{i_{i}^{\prime \prime}(\rho)+1} \cap \mathrm{Ult}(M ; E)$, the same functions with range $\subseteq V_{\rho+1}$ represent $x$ in $\operatorname{Ult}(M ; E)$ as in $\operatorname{Ult}(N ; E)$.

(Note that here, as always, we identify a wellfounded ultrapower with the isomorphic transitive set or class.)

Proof. (c) is obvious. (c) $\Rightarrow$ (a). (a) $\Rightarrow$ (b) since $Y \subseteq V_{i_{E^{\prime \prime}}^{\prime \prime}(\kappa)} \cap \mathrm{Ult}(M ; E)$.

Lemma 1.8. Let $M$ and $N$ be as in Lemma 1.6 and suppose that $N$ and $Y$, as well as $M$, are countably closed. Then $\operatorname{Ult}(N ; E)$, which is wellfounded by Lemma 1.6, is countably closed. (For arbitrary sets or classes such as $Y$, let us take countable closure to mean that every countable subset of $Y$ belongs to $Y$.) Proof. Suppose we are given $\left\langle\llbracket F_{i} \mathbb{1}_{a_{i}}^{N} \mid i \in \omega\right\rangle$. We must show that this sequence belongs to $\operatorname{Ult}(N ; E)$. Each $a_{i}$ belongs to $Y$, since $Y$ is countably closed. Hence $b=\left\langle a_{i} \mid i \in \omega\right\rangle \in Y$ by the countable closure of $Y$. Let $\widehat{F}:{ }^{\prime}\left(V_{\kappa}\right) \cap N \rightarrow$ $N$ be given by

$$
\hat{F}(\langle y\rangle)=\left\{\begin{array}{l}
\left\langle F_{i}(y(i)) \mid i \in \omega\right\rangle \quad \text { if } y: \omega \rightarrow N \&(\forall i)\left(y(i) \in^{l h\left(a_{i}\right)}\left(V_{\kappa}\right)\right) ; \\
\varnothing \text { otherwise. }
\end{array}\right.
$$

Each $F_{i} \in N$. Hence $\left\langle F_{i} \mid i \in \omega\right\rangle \in N$ by countable closure of $N$. Since $\ell h\left(a_{i}\right)$ is the $n$ such that domain $\left(F_{i}\right)={ }^{n}\left(V_{\kappa}\right) \cap N, \widehat{F} \in N$. Hence $\llbracket \widehat{F} \mathbb{1}_{\langle b\rangle}^{N} \in$ $\operatorname{Ult}(N ; E)$. Since $(E(\langle b\rangle))\left(\left\{\langle y\rangle \mid y: \omega \rightarrow N \&(\forall i)\left(y(i) \in^{\operatorname{lh}\left(a_{i}\right)}\left(V_{\kappa}\right)\right)\right\}\right)=1$ (essentially by Lemma 1.5 ), we have that $\llbracket \widehat{F} \rrbracket_{\langle b\rangle}^{N}$ is a function with domain $\omega$. Furthermore, $\llbracket \widehat{F} \mathbb{\rrbracket}_{\langle b\rangle}^{N}(i)=\llbracket F_{i} \rrbracket_{a_{i}}^{N}$ for each $i$, since

$$
\left.E\left(\langle b\urcorner^{\top} a_{i}\right)(\{\langle y\rangle\urcorner z \mid z=y(i)\}\right)=1 .
$$




\section{Homogeneous TREeS}

In this section we shall introduce the basic concepts needed to state our Main Theorem and prove some well-known facts about these concepts-facts that, however, do not seem to have been literally proved in any publication. We then deduce (assuming large cardinals) projective determinacy from these results and the Main Theorem (to be proved in $\S 5$ ). We also deduce $\mathrm{AD}^{L(\mathscr{R})}$ from these results, the Main Theorem, and a theorem of $\mathrm{H}$. Woodin.

A tree is a partially ordered set $(T, \prec)$ with the property that, for each $x \in T$, the set of all $y \prec x$ is wellordered by $\prec$. In descriptive set theory, the word "tree" is often used in a more special sense, for a set $T$ of finite sequences which is closed under initial segments. If we order such a $T$ by $x \prec y \Leftrightarrow x$ is properly extended by $y$, then $(T, \prec)$ is indeed a tree. Unfortunately it is also customary to order $T$ backward, letting $x \prec y$ mean that $x$ properly extends $y$. This is because the main interest is often in whether or not $T$ is wellfounded with respect to this backward partial ordering.

We adopt the following conventions.

(1) We use tree in the general sense (for a partially ordered set with wellordered initial segments).

(2) By a tree on a set $X$ we mean a subset $T$ of ${ }^{<\omega} X$ such that $(s \subseteq t \&$ $t \in T) \Rightarrow s \in T$, partially ordered by $s \prec t \Leftrightarrow t$ properly extends $s$. (Hence a tree on a set is literally a special kind of tree.) Since the partial ordering is determined by $T$, we usually speak of $T$ itself as a tree.

(3) We define other notions as in descriptive set theory: a tree $T$ on $X$ is wellfounded just in case $\succ$ is a wellfounded relation on $T$, i.e. just in case $T$ has no infinite branches, where an infinite branch of $T$ is an $f: \omega \rightarrow X$ such that each $x \mid n \in T .[T]$ is the set of all infinite branches of $T$. (So $T$ is wellfounded $\Leftrightarrow[T]=\varnothing$.) If $T$ is wellfounded, then we define inductively, for $t \in T$, the rank of $t$ in $T, \operatorname{rank}_{T}(t)$ by $\operatorname{rank}_{T}(t)=\sup \left\{\operatorname{rank}_{T}(s)+1 \mid s \in\right.$ $T \& t \subsetneq s\}$. The rank of $T$ is $\sup \left\{\operatorname{rank}_{T}(s)+1 \mid s \in T\right\}$.

When we deal with trees on cartesian products we shall always pretend that the trees consist of pairs of finite sequences instead of finite sequences of pairs: If $T$ is a tree on $X \times Y$ then we regard the members of $T$ as pairs $\langle s, t\rangle$ with $s \in{ }^{<\omega} X, t \in{ }^{<\omega} Y$, and $\ell h(s)=\ell h(t)$. Similarly we regard the members of [T] as pairs $\langle f, g\rangle$ with $f \in{ }^{\omega} X$ and $g \in{ }^{\omega} Y$.

If $A \in{ }^{\omega} X \times{ }^{\omega} Y, p A$, the projection of $A$, is $\{f:(\exists g)\langle f, g\rangle \in A\}$.

If $\kappa$ is a cardinal number and $B \subseteq{ }^{\omega} X, B$ is $\kappa$-Souslin if there is a tree $T$ on $X \times \kappa$ with $B=p[T]$.

We are primarily interested in subsets $B$ of ${ }^{\omega} \omega$. The axiom of choice is easily seen to give that every such $B$ is $2^{\aleph_{0}}$-Souslin. Proofs of determinacy from large cardinal axioms-including the proofs in this paper-typically depend on showing that the relevant set is not just $\kappa$-Souslin for some $\kappa$ but homogeneously Souslin. It is to this concept that we now turn. 
A tree $T$ on $Y \times Z$ is homogeneous if there is a system $\left\langle\mu_{s} \mid s \in{ }^{<\omega} Y\right\rangle$ such that

(1) each $\mu_{s}$ is a countably additive measure on $T_{s}=\{t \mid\langle s, t\rangle \in T\}$;

(2) if $s_{1} \subseteq s_{2}$, then $\mu_{s_{1}}(X)=1 \Leftrightarrow \mu_{s_{2}}\left(\left\{t|t| \ln \left(s_{1}\right) \in X\right\}\right)=1$;

(3) if $x \in p[T]$, then the ultrapower by $\left\langle\mu_{x \mid n} \mid n \in \omega\right\rangle$ is wellfounded.

(3) makes sense, as we can set $D=\omega, Z=Z$, and $\mu_{n}=\mu_{x \mid n}$, and then (2) asserts compatibility in the sense of $\S 1$.

Lemma 2.1. A tree $T$ on $Y \times Z$ is homogeneous just in case there are $\left\langle\mu_{s}\right| s \in$ $\left.{ }^{\omega} Y\right\rangle$ such that (1) and (2) hold and $\left(3^{\prime}\right)$ if $x \in p[T]$ and $\mu_{x \mid n}\left(X_{n}\right)=1$ for all $n \in \omega$, then there is an $f \in{ }^{\omega} Z$ with $(\forall n) f \uparrow n \in X_{n}$.

Proof. The lemma follows from Lemma 1.1.

Remark. There is a more general notion of homogeneous tree which has certain advantages and disadvantages. Replace $\left\langle\mu_{s} \mid s \in{ }^{<\omega} Y\right\rangle$ by $\left\langle\mu_{s} \mid s \in R\right\rangle$, where $R \subseteq{ }^{<\omega} Y$ is a tree on $Y$. Replace (3) by: if $x \in p[T]$ then $(\forall n)(x \uparrow n \in R)$ and the ultrapower by $\left\langle\mu_{x \mid n} \mid n \in \omega\right\rangle$ is wellfounded. All our results in this paper would go through with this liberalized definition. Among the advantages would be that it is a theorem of ZF that every closed subset $A$ of ${ }^{\omega} Y$ is $p[T]$ for some homogeneous (in the liberal sense) $T$. (Let $R=\{s|(\exists x)| s \subseteq x \& x \in A\}$. Let $T=\{\langle s, s\rangle \mid s \in R\}$. Let $\mu_{s}$ be the measure on $\{s\}$.) The disadvantageshaving to pay attention to $R$-are more important for us here.

A tree $T$ on $Y \times Z$ is $\kappa$-homogeneous if some $\left\langle\mu_{s} \mid s \in{ }^{<\omega} Y\right\rangle$ witnesses that $T$ is homogeneous with each $\mu_{s} \kappa$-complete. A set $A \subseteq{ }^{\omega} Y$ is homogeneously Souslin if $A=p[T]$ for some homogeneous $T . A$ is $\kappa$-homogeneously Souslin if $A=p[T]$ for some $\kappa$-homogeneous $T$.

The notion of homogeneous trees has its roots in [7], but the general notion was only isolated much later by Kechris [4] and Martin independently and was motivated in part by work of K. Kunen.

The following theorem provides the basic example of a homogeneous tree. It comes essentially from [7].

Theorem 2.2. If $A \subseteq{ }^{\omega} \omega$ is $\Pi_{1}^{1}$ and $\kappa$ is a measurable cardinal, then $A$ is $\kappa$-homogeneously Souslin.

Proof. As is well known, we can associate with each $s \in^{<\omega} \omega$ a linear ordering $<_{s}$ of $\ell h(s)$ in such a way that $s_{1} \subseteq s_{2} \Rightarrow<_{s_{1}} \subseteq<_{s_{2}}$, and hence that a linear ordering $<_{x}$ of $\omega$ is associated with each $x \in{ }^{\omega} \omega$, so that

$$
\left(\forall x \in{ }^{\omega} \omega\right)\left(x \in A \Leftrightarrow<_{x}\right. \text { is a wellordering). }
$$

In fact, the existence of such an association $s \mapsto<_{x}$ is equivalent with $A$ 's being $\Pi_{1}^{1}$.

Define a tree $T$ on $\omega \times \kappa$ by

$$
\begin{aligned}
T=\{ & \langle s, t\rangle \mid s \in{ }^{<\omega} \omega \& t \in{ }^{<\omega} \kappa \& \operatorname{lh}(s)=\ell h(t) \\
& \left.\&(\forall m<\operatorname{lh}(s))(\forall n<\operatorname{lh}(s))\left(m<_{s} n \Leftrightarrow t(m)<t(n)\right)\right\} .
\end{aligned}
$$


$A=p[T]$, since an $f$ with $\langle x, f\rangle \in[T]$ is just a verification that $<_{x}$ is a wellordering. (So far we have used only that $\kappa \geq \omega_{1}$.)

Let $\nu$ be a normal, $\kappa$-complete measure on $\kappa$. By Rowbottom's Theorem (see Theorem 70 of [3]), if $F:{ }^{n}[\kappa] \rightarrow\{0,1\}$, where $n \in \omega$, then there in an $X \subseteq \kappa$ with $\nu(X)=1$ and $\left.F\right|^{n}[X]$ constant. Thus we can define, for each $s \in{ }^{<\omega} \omega$, a measure $\mu_{s}$ on $T_{s}$ by

$\left.\mu_{s}(X)=1 \Leftrightarrow\left(\exists X^{\prime} \subseteq \kappa\right)\left(\nu\left(X^{\prime}\right)=1 \&(\forall t)\left(t \in T_{s} \& \operatorname{range}(t) \subseteq\left(X^{\prime}\right)\right) \Rightarrow t \in X\right)\right)$.

(Note that range $(t)$ determines $t$ for $t \in T_{s}$.)

Each $\mu_{s}$ is $\kappa$-complete, since $\nu$ is $\kappa$-complete. It is also easy to see that property (2) in the definition of homogeneous trees is enjoyed by $\left\langle\mu_{s} \mid s \in{ }^{<\omega} \omega\right\rangle$. To prove that $T$ is homogeneous, we must then verify property (3) and so, using Lemma 2.1 , we need only verify property $\left(3^{\prime}\right)$.

Let $X_{n} \subset T_{x \mid n}$ with $\mu_{x \mid n}\left(X_{n}\right)=1$ for each $n \in \omega$ and let $x \in A$. For each $n$, let $X_{n}^{\prime} \subseteq \kappa$ be such that $\nu\left(X_{n}^{\prime}\right)=1$ and $\left(t \in T_{x \mid n} \& \operatorname{range}(t) \subseteq X_{n}^{\prime}\right) \Rightarrow t \in$ $X_{n}$. Let $X=\bigcap_{n \in \omega} X_{n}^{\prime}$. Since $x \in A,<_{x}$ is a wellordering. Since $X$ is an uncountable set of ordinals, there is an $f: \omega \rightarrow X$ such that $(\forall m)(\forall n)\left(m<_{x}\right.$ $n \Leftrightarrow f(m)<f(n))$. For each $n, f \uparrow n \in T_{x \mid n}$ and $\operatorname{range}(f \uparrow n) \subseteq X \subseteq X_{n}^{\prime}$, so $f \mid n \in X_{n}$.

Remark. The theorem continues to hold-with essentially the same proof-if we let $A \subseteq{ }^{\omega} Y$ with $\neg A \aleph_{0}$-Souslin.

For our purposes, the most important property of homogeneously Souslin sets is determinacy. The following theorem is a standard fact in determinacy theory, but does not seem to have appeared in a published work. The theorem is a generalization of [7].

Theorem 2.3. Let $A \subseteq{ }^{\omega} Y$ be $\kappa$-homogeneously Souslin for some $\kappa>|Y| . A$ is determined.

Proof. Let $T$ on $Y \times Z$ be $\kappa$-homogeneous with $A=p[T]$. Let $G$ be the game given by $A$. Let $G^{*}$ be the game played as follows.

$$
\begin{array}{rlllll}
I & \left\langle y_{0}, z_{0}\right\rangle & & \left\langle y_{2}, z_{1}\right\rangle & & \cdots \\
I I & & y_{1} & & y_{3} & \cdots
\end{array}
$$

The rules are: Each $y_{i}$ must belong to $Y$. Each $z_{i}$ must belong to $Z$. Each $\left\langle\left\langle y_{i}\right| i\langle n\rangle,\left\langle z_{i} \mid i\langle n\rangle\right\rangle\right.$ must belong to $T$. The first player to disobey one of these rules loses. If all rules are obeyed, $I$ wins.

$G^{*}$ is a closed game and so is determined. If $I$ has a winning strategy for $G^{*}$, then $I$ has a winning strategy for $G$. (Just play the $y_{i}$ 's given by the strategy for $G^{*}$.)

Suppose then that $\tau^{*}$ is a winning strategy for $I I$ for $G^{*}$. Let $\left\langle\mu_{s} \mid s \in{ }^{<\omega} Y\right\rangle$ witness that $T$ is $\kappa$-homogeneous for some $\kappa>|Y|$. We now define a strategy $\tau$ for $I I$ for $G$. Let $\left\langle y_{i} \mid i \leq 2 n\right\rangle$ be a position in $G$ with $I I$ to move. Since 
$\mu_{\left\langle y_{i} \mid i \leq n\right\rangle}$ is $|Y|^{+}$-complete, there is a $y \in Y$ such that $\mu_{\left\langle y_{i} \mid i \leq n\right\rangle}\left(\left\{t \in{ }^{n+1} Z \mid \tau^{*}\right.\right.$ calls for $I I$ to play $y$ in the position given by $\left\langle y_{i} \mid i \leq 2 n\right\rangle$ and $\left.\left.t\right\}\right)=1$. Let $\tau$ call for $I I$ to play this $y$ in the position $\left\langle y_{i} \mid i \leq 2 n\right\rangle$.

Let $x \in{ }^{\omega} Y$ be a play of $G$ consistent with $\tau$. For each $n \in \omega$, let $X_{n}=$ $\left\{t \in{ }^{n+1} Z \mid \tau^{*}\right.$ calls for $I I$ to play $x(2 n+1)$ in the position given by $x \uparrow 2 n+$ 1 and $t\}$. By the definition of $\tau, \mu_{x \mid n+1}\left(X_{n}\right)=1$ for each $n$. Assume for a contradiction that $x \in A$. By Lemma 2.1, there is an $f \in{ }^{\omega} Z$ such that $(\forall n)|f| n+1 \in X_{n}$. But then $x$ and $f$ give a play of $G^{*}$ consistent with $\tau^{*}$ with all rules obeyed, contradicting the fact that $\tau^{*}$ is a winning strategy for II .

Our determinacy results will be proved via Theorem 2.3. In particular, we shall prove PD by showing - assuming Woodin cardinals-that every projective subset of ${ }^{\omega} \omega$ is homogeneously Souslin. To do this we shall use Theorem 2.3 and our Main Theorem, which will give us a method for propagating homogeneous Souslinness up the projective hierarchy.

A well-known fact (whose ultimate origin is [9]) - but which, like Theorem 2.3, does not seem to have been published (though see [4])-is that, if $B \subseteq{ }^{\omega} Y \times{ }^{\omega} \omega$ is homogeneously Souslin and $A=\neg p B$, i.e., $x \in A \Leftrightarrow$ $\left(\forall y \in{ }^{\omega} \omega\right)\langle x, y\rangle \notin B$, then $A$ is $\kappa$-Souslin for some $\kappa$ via a tree $\widetilde{T}$ constructed in a canonical fashion from a homogeneous $T$ with $B=p[T]$ plus measures witnessing the homogeneity of $T$. Our Main Theorem will say that under certain conditions $\widetilde{T}$ is $\eta$-homogeneous for certain $\eta$.

Shortly we shall define the operation giving $\widetilde{T}$, but first we shall describe a simpler operation, starting with a tree $T$ on $Y \times Z$ for some $Z$ and $\left\langle\mu_{s}\right|$ $\left.s \in{ }^{<\omega} Y\right\rangle$ witnessing the homogeneity of $T$, and giving a tree $T^{*}$ such that $p\left[T^{*}\right]=\neg p[T]$. There is a theorem simpler than the Main Theorem, giving that $T^{*}$ is under certain conditions $\eta$-homogeneous for certain $\eta$. In $\S 5$ we shall first present the proof of this simpler theorem, since all the ideas needed for the Main Theorem's proof appear in a more easily digested form in the proof of the simpler theorem.

We begin with the construction of $T^{*}$. Let $T$ be a tree on $Y \times Z$. Let $\left\langle\mu_{s} \mid s \in{ }^{<\omega} Y\right\rangle$ witness that $T$ is homogeneous. For $s \in{ }^{<\omega} Y$, let $j_{s}=i_{\mu_{s}}$ : $V \rightarrow M_{s}=\operatorname{Ult}\left(V ; \mu_{s}\right)$. For $s_{1} \subseteq s_{2}$, let $j_{s_{1}, s_{2}}: M_{s_{1}} \rightarrow M_{s_{2}}$ be given by $j_{s_{1}, s_{2}}\left(\llbracket F \prod_{\mu_{s_{1}}}\right)=\llbracket F^{\prime} \mathbb{1}_{\mu_{s_{2}}}$, where $F^{\prime}(t)=F\left(t \uparrow \operatorname{lh}\left(s_{1}\right)\right)$. As noted in $\S 1, j_{s_{1}, s_{2}}$ is well defined and is an elementary embedding. Define $T^{*}$ on $Y \times \mathrm{ON}$ by

$$
\begin{aligned}
\langle s, u\rangle \in T^{*} \Leftrightarrow & (\operatorname{lh}(s)=\operatorname{lh}(u) \& \\
& \left.\left(\forall i_{1}<\operatorname{lh}(s)\right)\left(\forall i_{2}<\operatorname{lh}(s)\right)\left(i_{1}<i_{2} \Rightarrow u\left(i_{2}\right)<j_{s\left\lceil i_{1}, s \mid i_{2}\right.}\left(u\left(i_{1}\right)\right)\right)\right) .
\end{aligned}
$$

Lemma 2.4.

$$
p\left[T^{*}\right]=p\left[T^{*} \uparrow\left(2^{|Z|}\right)^{+}\right]=\neg p[T],
$$

where $T^{*} \mid \alpha=\left\{\langle s, u\rangle \mid\langle s, u\rangle \in T^{*} \& u \in \epsilon^{<\omega} \alpha\right\}$. 
Proof. Suppose $x \in p\left[T^{*}\right]$. Let $\langle x, f\rangle \in\left[T^{*}\right]$. We have $j_{x\lceil i, x}: M_{x \uparrow i} \rightarrow M_{x}$, with $M_{x}$ the ultrapower by $\left\langle\mu_{x \mid i} \mid i \in \omega\right\rangle$, for each $i \in \omega$. Now

$$
j_{x\lceil i, x}(f(i))=j_{x\lceil i+1, x}\left(j_{x \nmid i, x \nmid i+1}(f(i))\right)>j_{x\lceil i+1, x}(f(i+1)) .
$$

Hence $\left\langle j_{x \uparrow i, x}(f(i)) \mid i \in \omega\right\rangle$ is an infinite descending sequence of ordinals in the ultrapower by $\left\langle\mu_{x \uparrow i} \mid i \in \omega\right\rangle$. Hence $x \notin p[T]$.

Suppose then that $x \notin p[T] . \quad T(x)=\left\{t \mid(\exists n)\left(t \in T_{x \mid n}\right)\right\}$ is a wellfounded tree. For $t \in T_{x \mid n}$, let $G_{n}(t)=\operatorname{rank}_{T(x)} t$. Let $f(n)=\llbracket G_{n} \rrbracket_{\mu_{x \mid n}}$. Since $G_{n+1}(t)<G_{n}(t \mid n)$ for all $t \in T_{x\lceil n+1}, f(n+1)=\llbracket G_{n+1} \rrbracket_{\mu_{x \uparrow n+1}}<$ $j_{x|n, x| n+1} \llbracket G_{n} \rrbracket_{\mu_{x \mid n}}=j_{x|n, x| n+1}(f(n))$. Hence $\langle x, f\rangle \in\left[T^{*}\right]$ and so $x \in$ $p\left[T^{*}\right]$.

If $Z$ is finite every $x$ belongs to $p[T]$. If $Z$ is infinite, then $|f(n)|=$ $\mid\left[\left|G_{n} \rrbracket_{x \mid n}\right| \leq\left|\left\{F \mid F:{ }^{n} Z \rightarrow \operatorname{rank}(T(x))\right\}\right| \leq|Z|^{|Z|}=2^{|Z|}\right.$. (This part of the lemma is not really important.)

Suppose now that $T$ is a tree on $(Y \times \omega) \times Z$ and that $\left\langle\mu_{\langle s, t\rangle}\right| s \in{ }^{<\omega} Y \& t \in$ $\left.{ }^{<\omega} \omega \& \ell h(s)=\ell h(t)\right\rangle$ witness that $T$ is homogeneous. Let $r_{0}, r_{1}, \ldots$ enumerate ${ }^{<\omega} \omega$ so that each finite sequence is enumerated before any of its proper extensions. Define a tree $\widetilde{T}$ on $Y \times$ ON by

$$
\begin{aligned}
\langle s, u\rangle \in \widetilde{T} & \Leftrightarrow\left(\forall i_{1}\right)\left(\forall i_{2}\right)\left[\left(i_{1}<i_{2}<\operatorname{lh}(s) \& r_{i_{1}} \subseteq r_{i_{2}}\right)\right. \\
& \left.\Rightarrow u\left(i_{2}\right)<j_{\left\langle\sinh \left(r_{i_{1}}\right), r_{i_{1}}\right\rangle,\left(\operatorname{silh}\left(r_{i_{2}}\right), r_{i_{2}}\right\rangle}\left(u\left(i_{1}\right)\right)\right],
\end{aligned}
$$

with the obvious definition of the $j_{\left\langle s_{1}, t_{1}\right\rangle,\left\langle s_{2}, t_{2}\right\rangle}$.

Lemma 2.5. $p[\widetilde{T}]=p\left[\widetilde{T} \uparrow\left(2^{|Z|}\right)^{+}\right]=\{x \mid(\forall y)\langle x, y\rangle \notin p[T]\}$.

Proof. Assume first that $x \in p[\tilde{T}]$. Let $(x, f) \in[\tilde{T}]$. Let $y \in{ }^{\omega} \omega$. Let $r_{i_{k}}=y \uparrow k$ for all $k \in \omega$. Then

$$
f\left(i_{k+1}\right)<j_{\langle x| k, y\lceil k\rangle,\langle x| k+1, y\lceil k+1\rangle}\left(f\left(i_{k}\right)\right)
$$

for all $k \in \omega$. As in the proof of Lemma 2.4, this gives us an infinite descending sequence of ordinals in the ultrapower by $\left\langle\mu_{\langle x|n, y| n\rangle} \mid n \in \omega\right\rangle$. Hence $\langle x, y\rangle \notin$ $p[T]$.

Now suppose that $\left(\forall y \in{ }^{\omega} \omega\right)\langle x, y\rangle \notin p[T]$. Let $S=T(x)=\{\langle r, t\rangle \mid$ $\langle\langle x \mid \ell h(r), r\rangle, t\rangle \in T\}$. $S$ can have no infinite branches, i.e. $S$ is wellfounded. Let $G_{i}(t)=\operatorname{rank}_{S}\left(\left\langle r_{i}, t\right\rangle\right)$ and let $g(i)=\llbracket G_{i} \rrbracket_{\mu_{\left(x, t h\left(r_{i}\right), r_{i}\right\rangle}}$. As in the proof of Lemma 2.4, if $r_{i} \subsetneq r_{j}$ then $G_{j}(t)<G_{i}\left(t \uparrow \operatorname{lh}\left(r_{i}\right)\right)$ for all $t \in$ $T_{\left\langle x \mid \ell h\left(r_{j}\right), r_{j}\right\rangle}$. As in the proof of Lemma 2.4, this implies that $g(j)<$ $j_{\left\langle x \mid \ell h\left(r_{i}\right), r_{i}\right\rangle,\left\langle x \mid \ell h\left(r_{j}\right), r_{j}\right\rangle}(g(i))$ and so that $\langle x, g\rangle \in[\widetilde{T}]$. Thus $x \in p[\widetilde{T}]$.

Also as in the proof of Lemma 2.4, $g(i)<\left(2^{|Z|}\right)^{+}$for each $i$.

In order to state our Main Theorem, we must formulate our large cardinal hypothesis. This means we must define the relevant kind of large cardinals: Woodin cardinals. In this section we shall give only the bare definition. Woodin 
cardinals will be studied in $\S 4$ and will be related to more familiar kinds of large cardinals. A cardinal $\delta$ is Woodin if, for every $f: \delta \rightarrow \delta$, there is a $\kappa<\delta$ such that $\kappa$ is closed under $f$ and such that there is an elementary embedding $j: V \rightarrow M$ with $\operatorname{crit}(j)=\kappa, M$ transitive, and $V_{(j(f))(\kappa)} \in M$.

Main Theorem (Theorem 5.11). Let $\delta$ be a Woodin cardinal, let $\left\langle\mu_{\langle s, r\rangle}\right| s \in$ $\left.{ }^{<\omega} \omega \& r \in{ }^{<\omega} \omega \& \ell h(s)=\ell h(r)\right\rangle$ witness that the tree $T$ on $(\omega \times \omega) \times Z$ is $\delta^{+}$-homogeneous, and let $\widetilde{T}$ be defined as above. For each $\alpha<\delta, \widetilde{T}$ is $\alpha$-homogeneous.

Remarks. (1) The measures witnessing the $\alpha$-homogeneity of $\tilde{T}$ for various $\alpha<\delta$ all concentrate in some sufficiently large ${ }^{<\omega} \beta$. Hence they witness that the set $\tilde{T} \uparrow \beta$ is $\alpha$-homogeneous.

(2) The theorem remains true-and our proof continues to work with essentially no change-if we let $T$ be a tree on $(Y \times \omega) \times Z$ for any $Y \in V_{\delta}$.

Corollary. For each $n \in \omega$, if there is a measurable cardinal larger than $n$ Woodin cardinals, then $\left(\boldsymbol{\Pi}_{n+1}^{1}\right)$ determinacy holds.

Proof. Let $\delta_{1}<\delta_{2}<\cdots<\delta_{n}$ be Woodin cardinals if $n>0$. Let $\delta_{0}=\omega$. Let $\rho>\delta_{n}$ be measurable. Let $\delta_{i}<\alpha_{i}<\delta_{i+1}$ for $i<n$ and let $\alpha_{n}=\rho$. By induction we show that every $\Pi_{i+1}^{1}$ set is $\alpha_{n-i}$-homogeneously Souslin, for $0 \leq i \leq n$.

For the case $i=0$, Theorem 2.2 gives that every $\Pi_{1}^{1}$ set is $\rho$-homogeneously Souslin. $\alpha_{n-0}=\alpha_{n}=\rho$.

Assume that every $\boldsymbol{\Pi}_{i+1}^{1}$ set is $\alpha_{n-i}$-homogeneously Souslin, for $0 \leq i<n$. Let $A \subseteq{ }^{<\omega} \omega$ be $\boldsymbol{\Pi}_{i+2}^{1}$. Then $A=\{x \mid(\forall y)(x, y) \notin B\}$ with $B \in \boldsymbol{\Pi}_{i+1}^{1}$. Let $T$ on $(\omega \times \omega) \times Z$ for some $Z$ witness that $B$ is $\alpha_{n-i}$-homogeneously Souslin. Since $\delta_{n-i}<\alpha_{n-i}$ and $\alpha_{n-(i+1)}<\delta_{n-i}$, the Main Theorem implies that $\widetilde{T}$ (and so $\widetilde{T} \mid \beta$ for some $\beta$ ) is $\alpha_{n-(i+1)}$-homogeneous. By Theorem 2.5, $p[\tilde{T}]=A$ and hence $A$ is $\alpha_{n-(i+1)}$-homogeneously Souslin.

The case $i=n$ gives us that every $\Pi_{n+1}^{1}$ set is homogeneously Souslin and so, by Theorem 2.3, determined.

More determinacy can be deduced from a combination of the Main Theorem and results of $\mathrm{H}$. Woodin.

Theorem (Woodin). If there is a measurable cardinal larger than infinitely many Woodin cardinals, then every subset of ${ }^{\omega} \omega$ in $L(\mathscr{R})$ is of the form $\{x \mid(\forall y)(x, y)$ $\notin p[T]\}$ for some tree on $(\omega \times \omega) \times Z$, for some $Z$, such that $T$ is $\delta^{+}$-homogeneous for some Woodin cardinal $\delta$.

Corollary (to the Woodin Theorem and the Main Theorem). If there is a measurable cardinal larger than infinitely many Woodin cardinals, then every subset of ${ }^{\omega} \omega$ in $L(\mathscr{R})$ is determined (hence the Axiom of Determinacy holds in $L(\mathscr{R})$ ).

The corollary follows directly from the Woodin Theorem, the Main Theorem, and Theorem 2.3. Assuming the existence of more Woodin cardinals, 
Woodin can strengthen the conclusion of his theorem, replacing $L(\mathscr{R})$ by larger classes. The conclusion of the corollary is correspondingly strengthened. From the stronger hypothesis that a supercompact cardinal exists, Woodin proves the conclusion of his theorem in [13]. In [13] Woodin states his conclusion in terms of weakly homogeneous trees. For our purposes a weakly homogeneous tree is a tree on $Y \times(\omega \times Z)$ such that the corresponding tree on $(Y \times \omega) \times Z$ is homogeneous.

Embedding normal form. If a tree $T$ on $Y \times Z$ is homogeneous and $A=p[T]$, then there is a system $\left(\left\langle M_{s} \mid s \in{ }^{<\omega} Y\right\rangle,\left\langle k_{s_{1}, s_{2}} \mid s_{1} \subseteq s_{2} \& s_{1}, s_{2} \in{ }^{<\omega} Y\right\rangle\right)$ such that

(a) $M_{0}=V$ and each $M_{s}$ is a transitive proper class model of ZFC.

(b) $k_{s_{1}, s_{2}}: M_{s_{1}} \rightarrow M_{s_{2}}$ is elementary and $\left(s_{1} \subseteq s_{2} \subseteq s_{3} \Rightarrow k_{s_{1}, s_{3}}=k_{s_{2}, s_{3}} \circ\right.$ $\left.k_{s_{1}, s_{2}}\right)$.

(c) if $x \in{ }^{\omega} Y$ and $\left(M_{x},\left\langle k_{x \mid n, x} \mid n \in \omega\right\rangle\right)$ is the direct limit of the system $\left(\left\langle M_{x \mid n} \mid n \in \omega\right\rangle,\left\langle k_{x|m, x| n} \mid m \leq n \in \omega\right\rangle\right)$, then $x \in A \Leftrightarrow M_{x}$ is wellfounded.

(Just let $\left\langle\mu_{s} \mid s \in{ }^{<\omega} Y\right\rangle$ witness that $T$ is homogeneous, and let $k_{s_{1}, s_{2}}=$ $j_{s_{1}, s_{2}}: M_{s_{1}} \rightarrow M_{s_{2}}$, where $M_{s}=\operatorname{Ult}\left(V ; \mu_{s}\right)$ and $j_{s_{1}, s_{2}}$ is the canonical elementary embedding.)

Let us say that $\left(\left\langle M_{s} \mid s \in{ }^{<\omega} Y\right\rangle,\left\langle k_{s_{1}, s_{2}} \mid s_{1} \subseteq s_{2} \& s_{1}, s_{2} \in{ }^{<\omega} Y\right\rangle\right)$ gives an embedding normal form for $A$ if (a), (b), and (c) hold and that $A$ has an embedding normal form if some system gives an embedding normal form for $A$.

In proving the Main Theorem, it will help our motivation if we aim directly for an embedding normal form for $\{x \mid(\forall y)(x, y) \notin p[T]\}$, rather than for full homogeneity of $\widetilde{T}$. Once we get embedding normal form, we will see that our method for doing so also gives homogeneity.

\section{ITERATION TREES}

In order to prove (under the hypotheses of the Main Theorem) that $p[\widetilde{T}]$ has an embedding normal form-and that the conclusion of the Main Theorem holds-we shall associate with each $x \in{ }^{\omega} \omega$ not just a single sequence of elementary embeddings but rather a whole tree of elementary embeddings. One branch of this tree will provide us with the sequence of embeddings which give an embedding normal form. We need the rest of the tree to be able to control the wellfoundedness of the direct limit model along the main branch.

For $n \in \omega$, an iteration tree on $V$ of length $n+1$ is a system

$$
\left(\prec,\left\langle M_{k} \mid k \leq n\right\rangle,\left\langle E_{k} \mid k<n\right\rangle,\left\langle\rho_{k} \mid k<n\right\rangle\right)
$$

with the following properties:

(1) $(n+1, \prec)$ is a tree with 0 the $\prec$-least element, and $\prec$ respects the natural ordering on $n+1$. 
(2) Each $M_{k}$ is a transitive proper class model of ZFC and $M_{0}=V$.

(3) $\left\langle\rho_{k}\right| k\langle n\rangle$ is a nondecreasing sequence of ordinals.

(4) $k_{1} \leq k_{2} \leq n \Rightarrow V_{\rho_{k_{1}}+1} \cap M_{k_{1}}=V_{\rho_{k_{1}}+1} \cap M_{k_{2}}$.

(5) $M_{k}$ satisfies " $E_{k}$ is an extender" (so, in particular, $E_{k} \in M_{k}$ ).

(6) $\operatorname{Support}\left(E_{k}\right) \supseteq V_{\rho_{k}+1} \cap M_{k}$.

(7) Let $k^{*}$ be the immediate predecessor of $k+1$ with respect to $\prec$, for $k<n . \rho_{k^{*}} \geq \operatorname{crit}\left(E_{k}\right)$ and $M_{k+1}=\operatorname{Ult}\left(M_{k^{*}} ; E_{k}\right)$.

Remarks. (a) Our definition is almost the same as that of [11]. In [11] only extenders with ordinal supports are considered. This necessitates a change in (6). The natural replacement is

(6 $\left.{ }^{\prime}\right) V_{\rho_{k}+1} \cap M_{k} \subseteq \operatorname{Ult}\left(M_{k} ; E_{k}\right)$.

Allowing only ordinal supports and replacing (6) by $\left(6^{\prime}\right)$ makes no real change in the concept: For any iteration tree in one of the two senses, there is an iteration tree in the other sense with the same models $M_{k}$, the same ordinals $\rho_{k}$, and the same elementary embeddings of $M_{k^{*}}$ into $M_{k+1}$. In [11], however, the clause corresponding to (6) (in the final official definition) is not $\left(6^{\prime}\right)$ but rather

$$
V_{\rho_{k}+2} \cap M_{k} \subseteq \operatorname{Ult}\left(M_{k} ; E_{k}\right) .
$$

The reason for this is certain technical problems connected with iteration trees of transfinite length. We shall not consider such trees here, so we keep the more natural $\rho_{k}+1$.

(b) In [11] iteration trees not on $V$ are considered. There not only the condition $M_{0}=V$ is dropped but also the condition that the $M_{k}$ be proper classes is dropped and the condition that they be models of ZFC is weakened.

(c) Note that (4) guarantees that (7) makes sense-that there is an $\operatorname{Ult}\left(M_{k^{*}} ; E_{k}\right)$. ((4) actually follows from the other clauses.)

(d) The possibility that superstrong cardinals might generate complicated iteration trees arose as a worry in Steel's work on inner models for large cardinal axioms (see [11]).

(e) Implicit in (7) is the requirement that $\operatorname{Ult}\left(M_{k^{*}} ; E_{k}\right)$ be wellfounded. In fact, it can be proved that this is automatically the case. See [11]. Here we shall avoid having to prove this by considering only "countably closed" iteration trees.

An iteration tree on $V$ of length $n+1$ is countably closed if each $M_{k}$ satisfies "support $\left(E_{k}\right)$ is countably closed." By Lemma 1.8, this implies-and so is equivalent with-the countable closure of all the $M_{k}$ and all the $\operatorname{support}\left(E_{k}\right)$.

(f) Note that $\left(\prec,\left\langle E_{k} \mid k<n\right\rangle,\left\langle\rho_{k} \mid k<n\right\rangle\right)$ completely determine the iteration tree. If we were being careful about sets versus classes, we would have defined this set to be the tree.

Lemma 3.1. Let $\left(\prec,\left\langle M_{k} \mid k \leq n\right\rangle,\left\langle E_{k} \mid k<n\right\rangle,\left\langle\rho_{k} \mid k<n\right\rangle\right)$ be a countably closed iteration tree on $V$ of length $n+1$. Let $E \in M_{n}$ and $\rho \geq \rho_{n-1}$, if $n>0$, be such that $M_{n}$ satisfies " $E$ is an extender with countably closed support and $\operatorname{support}(E) \supseteq V_{\rho+1}$." Suppose $\tilde{n} \leq n$ is such that $\operatorname{crit}(E) \leq \rho_{\tilde{n}}$. There 
is a unique countably closed iteration tree $\left(\prec^{\prime},\left\langle M_{k}^{\prime}\right| k \leq n+1\right),\left\langle E_{k}^{\prime}\right| k<$ $\left.n+1\rangle,\left\langle\rho_{k}^{\prime} \mid k<n+1\right\rangle\right)$ on $V$ of length $n+2$ with $M_{k}^{\prime}=M_{k}$ for all $k \leq n$, $\rho_{k}^{\prime}=\rho_{k}$, and $E_{k}^{\prime}=E_{k}$ for $k<n, \prec^{\prime} \mid n+1=\prec, E_{n}^{\prime}=E, \rho_{n}^{\prime}=\rho$, and $\tilde{n}$ the immediate predecessor on $n+1$ with respect to $\prec^{\prime}$.

Proof. Let $\prec^{\prime} \mid n+1=\prec$ and let $k \prec^{\prime} n+1 \Leftrightarrow k \preceq \tilde{n}$. By (4), the critical point $\kappa$ of $E$ satisfies $V_{\kappa+1} \cap M_{\tilde{n}}=V_{\kappa+1} \cap M_{n}$. Thus we can form $\operatorname{Ult}\left(M_{\tilde{n}} ; E\right)$. By Lemma 1.6 and the fact that $M_{n}$ is countably closed, we get that $M_{n+1}^{\prime}=$ $\operatorname{Ult}\left(M_{\tilde{n}} ; E\right)$ is wellfounded. By Lemma 1.7, $V_{\rho_{n}+1} \cap M_{n+1}=V_{\rho_{n}+1} \cap M_{n}$. Thus (4) holds with $k_{2}=n+1$.

An iteration tree on $V$ of length $\omega$ is a system

$$
\mathscr{T}=\left(\prec,\left\langle M_{k} \mid k \in \omega\right\rangle,\left\langle E_{k} \mid k \in \omega\right\rangle,\left\langle\rho_{k} \mid k \in \omega\right\rangle\right)
$$

such that each $\mathscr{T}_{n}=\left(\langle| n+1,\left\langle M_{k} \mid k \leq n\right\rangle,\left\langle E_{k} \mid k<n\right\rangle,\left\langle\rho_{k} \mid k<n\right\rangle\right)$ is an iteration tree on $V$ of length $n+1$. $\mathscr{T}$ is countably closed if each $\mathscr{T}_{n}$ is countably closed.

Let $\mathscr{T}=\left(\prec,\left\langle M_{k} \mid k<\alpha\right\rangle,\left\langle E_{k} \mid k+1<\alpha\right\rangle,\left\langle\rho_{k} \mid k+1<\alpha\right\rangle\right)$ be an iteration tree on $V$ of length $\alpha$ with $0<\alpha \leq \omega$. The canonical elementary embeddings associated with $\mathscr{T}$ are the $i_{k_{1}, k_{2}}$ for $k_{1} \preceq k_{2}<\alpha$, defined as follows.

(i) $i_{k_{1}, k_{1}}$ is the identity: $M_{k_{1}} \rightarrow M_{k_{1}}$.

(ii) $i_{k^{*}, k+1}=i_{E_{k}}^{M_{k^{*}}}: M_{k^{*}} \rightarrow M_{k+1}$.

(iii) $i_{k_{1}, k_{3}}=i_{k_{2}, k_{3}} \circ i_{k_{1}, k_{2}}$ for $k_{1} \preceq k_{2} \preceq k_{3}$.

A branch of an iteration tree on $V$ of length $\alpha \leq \omega$ is a maximal linearly ordered subset of $\omega$ with respect to the $\prec$ of the iteration tree. An infinite branch of an iteration tree on $V$ of length $\omega$ is then an infinite, maximal linearly ordered $(\prec)$ subset of $\omega$. If $b$ is a branch of an iteration tree, we can form the direct limit

$$
\left(M_{b},\left\langle i_{k, b} \mid k \in b\right\rangle\right)
$$

of the directed system $\left(\left\langle M_{k} \mid k \in b\right\rangle,\left\langle i_{k_{1}, k_{2}} \mid k_{1} \preceq k_{2} \in b\right\rangle\right)$. In order to get embedding normal form results, we would like to be able to build iteration trees with infinite branches $b$ so that we can control whether or not $M_{b}$ is wellfounded. It is easier to guarantee that an $M_{b}$ is illfounded than that it is wellfounded. (Just build in an infinite descending sequence of "ordinals" of $M_{b}$.) Lemma 3.2 will help us to deal with this problem. What Lemma 3.2 says is that, if all infinite branches other than $b$ are illfounded and if this is witnessed in a sufficiently continuous fashion, then $M_{b}$ is wellfounded.

Lemma 3.2. Let $\mathscr{T}=\left(\prec,\left\langle M_{n} \mid n \in \omega\right\rangle,\left\langle E_{n} \mid n \in \omega\right\rangle,\left\langle\rho_{n} \mid n \in \omega\right\rangle\right)$ be a countably closed iteration tree on $V$ of length $\omega$ and let $b$ be an infinite branch of $\mathscr{T}$. Suppose that there are ordinal numbers $\left\langle\xi_{n} \mid n \in \omega-b\right\rangle$ such that, for each $n$ with $n^{*} \notin b, \xi_{n+1}<i_{n^{*}, n+1}\left(\xi_{n^{*}}\right)$ (i.e. $\xi_{n+1}<i_{E_{n}}^{M_{n^{*}}}\left(\xi_{n^{*}}\right)$ ). Then $M_{b}$ is wellfounded.

Proof. Let $i_{m, n}, m \preceq n$, be the canonical elementary embeddings associated with our iteration tree. Let $\gamma$ be a limit ordinal such that, for each $n$, 
$\operatorname{support}\left(E_{n}\right) \in V_{i_{0, n}(\gamma)}$. We may assume without loss of generality that each $\xi_{n}$ for $n \notin b$ is in $M_{n}$ a limit ordinal of cofinality $>i_{0, n}(\gamma)$ with $V_{\xi_{n}}$ satisfying $\Sigma_{2}$-replacement. (Just replace the given $\xi_{n}$ by the $\xi_{n}$ th limit ordinal $\xi$ of cofinality $>i_{0, n}(\gamma)$ such that $V_{\xi}$ satisfies $\Sigma_{2}$-replacement in $M_{n}$.)

Assume for a contradiction that $M_{b}$ is illfounded. By Lemma 1.2(a) let $\left\langle\xi_{n} \mid n \in b\right\rangle$ be such that $\xi_{n+1}<i_{E_{n}}^{M_{n^{*}}}\left(\xi_{n^{*}}\right)$ for each $n$ such that $n+1 \in b$.

Our plan is to replace $\left(\left\langle M_{n} \mid n \in b\right\rangle,\left\langle i_{m, n} \mid m \preceq n \in b\right\rangle\right)$ by an internal iteration $\left(\left\langle\bar{M}_{n} \mid n \in b\right\rangle,\left\langle\bar{i}_{m, n} \mid m \preceq n \in b\right\rangle\right)$. We shall have elementary embeddings $\pi_{n}: \bar{M}_{n} \rightarrow M_{n}$, embeddings that commute with the embeddings of the two iterations. Furthermore, we shall arrange that each $\xi_{n}$, for $n \in b$, is in the range of the corresponding $\pi_{n}$. This will give us the contradiction that the direct limit of the internal iteration is not wellfounded. To get an internal iteration, we replace the natural candidates for the $\bar{M}_{n}, n \notin b$, by models which belong to earlier models $\bar{M}_{m}$. This is done by taking Skolem hulls of ranks in the natural candidates. The $\xi_{n}, n \notin b$, will give us the ranks in which to take these hulls. Note that we are not building a new iteration tree, but only a kind of approximation to one.

We begin by defining a sequence of countable sets which contain the important elements of the $M_{n}$. For each $d \in M_{n+1}$, choose $f_{n}^{d} \in M_{n *}$ and $a_{n}^{d} \in M_{n}$ such that $d=\llbracket f_{n}^{d} \mathbb{1}_{a_{n}^{d}, E_{n}}^{M_{n^{*}}}$, making sure that $f_{n}^{d}$ has minimal possible rank. For $m, n \in \omega$ we shall define, by induction on $m$, subsets $C_{n}^{m}$ of $M_{n}$. Set $C_{n}^{0}=\left\{\xi_{n}, E_{n}, \rho_{n}\right\}$. If $C_{n}^{m}$ has been defined for each $n$, let

$$
C_{n}^{m+1}=C_{n}^{m} \cup\left\{a_{n}^{d} \mid d \in C_{n+1}^{m}\right\} \cup\left\{f_{n^{\prime}}^{d} \mid d \in C_{n^{\prime}+1}^{m} \& n=\left(n^{\prime}\right)^{*}\right\} .
$$

Now let $C_{n}=\bigcup_{m \in \omega} C_{n}^{m}$. We shall see that

(i) each $C_{n}$ is a countable subset of $M_{n}$;

(ii) for each $n,\left\{\xi_{n}, E_{n}, \rho_{n}\right\} \subseteq C_{n}$;

(iii) whenever $d \in C_{n+1}$, there are $a \in C_{n}$ and $f \in C_{n^{*}}$ such that $d=$ 【f $\prod_{a, E_{n}}^{M_{n^{*}}}$

(iv) for each $n \in \omega-b, C_{n}-\left\{\xi_{n}\right\} \subseteq V_{\xi_{n}}$; for each $n$ and each $d \in C_{n+1}$, if $n \notin b$ then $a_{n}^{d} \in V_{\xi_{n}}$ and if $n^{*} \notin b$ then $f_{n}^{d} \in V_{\xi_{n^{*}}}$.

(i), (ii), and (iii) are obvious. For (iv) we proceed by induction on $m$, showing that $C_{n}^{m}-\left\{\xi_{n}\right\} \subseteq V_{\xi_{n}}$ for each $n \notin b$. For $m=0$ and $n \notin b$ we have that $\operatorname{support}\left(E_{n}\right) \in V_{i_{0, n}(\gamma)} \subseteq V_{\xi_{n}}$ and that $V_{\rho_{n}} \cap M_{n} \subseteq \operatorname{support}\left(E_{n}\right)$, the latter by the definition of iteration tree. Since $\xi_{n}$ is a limit ordinal, these facts imply $E_{n} \in V_{\xi_{n}}$ and $\rho_{n} \in V_{\xi_{n}}$. Now let us consider the induction step, for $m+1$. It suffices to show, for $d \in C_{n+1}^{m}$, that if $n \notin b$ then $a_{n}^{d} \in V_{\xi_{n}}$ and if $n^{*} \notin b$ then $f_{n}^{d} \in V_{\xi_{n^{*}}}$. Now $a_{n}^{d} \in{ }^{<\omega}\left(\operatorname{support}\left(E_{n}\right)\right) \in V_{\xi_{n}}$ for $n \notin b$. Assume $n^{*} \notin b$. By induction we have that $d \in V_{\xi_{n+1}+1}$. Since $d=\llbracket f_{n}^{d} \prod_{a_{n}^{d}, E_{n}}^{M_{*}^{*}}$ this means that $\llbracket f_{n}^{d} \rrbracket_{a_{n}^{d}, E_{n}} \in V_{\xi_{n+1}+1} \subseteq V_{i_{E_{n}}^{1 n^{*}}\left(\xi_{n^{*}}\right)}$ by the fact that $\xi_{n+1}<i_{E_{n}}^{M_{n^{*}}}\left(\xi_{n^{*}}\right)$. By the 
minimal rank constraint on the choice of $f_{n}^{d}$, this implies that $f_{d}^{n}(z) \in V_{\xi_{n^{*}}}$ for every $z$. Since $\xi_{n^{*}}>\rho_{n^{*}} \geq \operatorname{crit}\left(E_{n}\right)$ and since $\xi_{n^{*}}$ is a limit ordinal of cofinality $>i_{0, n}(\gamma)$ we get that $f_{n}^{d} \in V_{\xi_{n^{*}}}$ as desired.

We next turn to the main construction of the proof of the lemma, in which we replace the iteration along $b$ by an internal iteration which nevertheless has illfounded direct limit.

We shall define, by induction on $n,\left\langle\bar{M}_{n} \mid n \in \omega\right\rangle,\left\langle\bar{E}_{n} \mid n \in \omega\right\rangle,\left\langle\bar{\rho}_{n}\right| n \in$ $\omega\rangle$, and $\left\langle\pi_{n} \mid n \in \omega\right\rangle$ such that

(a) for each $n \in b, \bar{M}_{n}$ is a countably closed transitive proper class model of ZFC;

(b) for each $n \in \omega-b, \bar{M}_{n}$ is a countably closed transitive set model of $\mathrm{ZC}+\Sigma_{2}$-replacement (see $\left.\S 1\right)$

(c) for each $n \in b, \pi_{n}: \bar{M}_{n} \rightarrow M_{n}$ is an elementary embedding with $C_{n} \subseteq \operatorname{range}\left(\pi_{n}\right)$

(d) for each $n \in \omega-b, \pi_{n}: \bar{M}_{n} \rightarrow V_{\xi_{n}} \cap M_{n}$ is an elementary embedding with $C_{n}-\left\{\xi_{n}\right\} \subseteq \operatorname{range}\left(\pi_{n}\right)$;

(e) for each $n, \pi_{n}\left(\bar{\rho}_{n}\right)=\rho_{n}$;

(f) each $\bar{M}_{n}$ satisfies " $\bar{E}_{n}$ is an extender with countably closed support and support $\left(\bar{E}_{n}\right) \supseteq V_{\bar{\rho}_{n+1}}$ ";

(g) $\bar{M}_{n+1}=\operatorname{Ult}\left(\bar{M}_{n^{*}} ; \bar{E}_{n}\right)$ for each $n$ such that $n+1 \in b$;

(h) for each $n$ with $n+1 \in b$, we have the following commutative diagram:

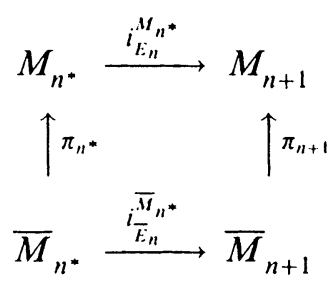

(i) for each $n, V_{\bar{\rho}_{n}+1} \cap \bar{M}_{n}=V_{\bar{\rho}_{n}+1} \cap \bar{M}_{m}$ for every $m \geq n$;

(j) for each $n, \pi_{n} \uparrow V_{\bar{\rho}_{n}+1} \cap \bar{M}_{n}=\pi_{m} \uparrow V_{\bar{\rho}_{n}+1} \cap \bar{M}_{m}$, for every $m \geq n$;

(k) for each $n \in \omega-b, \bar{M}_{n} \in \bar{M}_{\hat{n}}$, where $\hat{n}$ is the largest element of $b$ smaller than $n$ (in the natural order of $\omega$ ).

Let $\bar{M}_{0}=V$ and let $\pi_{0}: V \rightarrow V$ be the identity.

Assume that $\left\langle\bar{M}_{k} \mid k \leq n\right\rangle,\left\langle\bar{E}_{k} \mid k<n\right\rangle,\left\langle\bar{\rho}_{k} \mid k<n\right\rangle$, and $\left\langle\pi_{k} \mid k \leq n\right\rangle$ are defined and satisfy $(\mathrm{a})-(\mathrm{k})$.

Let us consider first the case that $n+1 \in b$. By (c) and (d) and property (ii) of $C_{n}$, we may define $\bar{E}_{n}$ and $\bar{\rho}_{n}$ by $\pi_{n}\left(\bar{E}_{n}\right)=E_{n}$ and $\pi_{n}\left(\bar{\rho}_{n}\right)=\rho_{n}$. (e) holds by definition. If $n \in b$ the elementarity of $\pi_{n}: \bar{M}_{n} \rightarrow M_{n}$ ((c)) gives (f). If $n \notin b$ then $\pi_{n}: \bar{M}_{n} \rightarrow V_{\xi_{n}} \cap M_{n}$ is elementary, and so we need only check that $V_{\xi_{n}} \cap M_{n}$ satisfies " $E_{n}$ is an extender with countably closed support and support $\left(E_{n}\right) \supseteq V_{\rho_{n}+1}$." But the definition of iteration tree implies that $M_{n}$ satisfies this formula and the formula is absolute in $M_{n}$ for limit ranks. 
$\operatorname{crit}\left(E_{n}\right) \leq \rho_{n^{*}}$, by the definition of iteration tree, and $\pi_{n^{*}}\left(\bar{\rho}_{n^{*}}\right)=\pi_{n}\left(\bar{\rho}_{n^{*}}\right)=$ $\rho_{n^{*}}$, so $\operatorname{crit}\left(\bar{E}_{n}\right) \leq \bar{\rho}_{n^{*}}$. Therefore (i) guarantees that $V_{\operatorname{crit}\left(\bar{E}_{n}\right)+1} \cap \bar{M}_{n^{*}}=$ $V_{\text {crit }\left(\bar{E}_{n}\right)+1} \cap \bar{M}_{n}$. We may then set $\bar{M}_{n+1}=\operatorname{Ult}\left(\bar{M}_{n^{*}} ; \bar{E}_{n}\right)$. We have the elementary embedding $i \bar{E}_{\bar{M}_{n}}: \bar{M}_{n^{*}} \rightarrow \bar{M}_{n+1}$. By (a), (b), (d), and (f), Lemma 1.6 gives that $\bar{M}_{n+1}$ is wellfounded, and Lemma 1.8 gives that it is countably closed. Hence (a) holds for $n+1$.

Define $\pi_{n+1}: \bar{M}_{n+1} \rightarrow M_{n+1}$ by

$$
\pi_{n+1}\left(\llbracket f \mathbb{1}_{a, \bar{E}_{n}}^{\bar{M}_{n}}\right)=\llbracket \pi_{n^{*}}(f) \prod_{\pi_{n}(a), E_{n}}^{M_{n^{*}}} .
$$

To see that $\pi_{n+1}$ is well defined, let $a, b \in^{<\omega}\left(\operatorname{support}\left(\bar{E}_{n}\right)\right)$, let $m=\ell h(a)$, and let $m^{\prime}=\ell h(b)$.

$$
\begin{aligned}
& \llbracket f \rrbracket_{a, \bar{E}_{n}^{*}}^{\bar{M}_{n}^{*}}=\llbracket g \rrbracket_{b, \bar{E}_{n}^{*}}^{\bar{M}_{n}^{*}} \\
& \Leftrightarrow\left(\bar{E}_{n}(\hat{a} b)\right)\left(\left\{z \mid f(z \mid m)=g\left(\left\langle z(m+k) \mid k<m^{\prime}\right\rangle\right)\right\}\right)=1 \\
& \Leftrightarrow\left(\pi_{n}\left(\bar{E}_{n}\left(a^{-} b\right)\right)\right)\left(\pi_{n}\left(\left\{z \mid f(z \mid m)=g\left(\left\langle z(m+k) \mid k<m^{\prime}\right\rangle\right)\right\}\right)\right)=1 \\
& \Leftrightarrow\left(\text { since } \pi_{n} \text { and } \pi_{n^{*}} \text { agree on } V_{\bar{\rho}_{n^{*}+1}} \cap \bar{M}_{n^{*}} \text { and since } \operatorname{crit}\left(\bar{E}_{n}\right) \leq \bar{\rho}_{n^{*}}\right) \\
& \left(\pi_{n}\left(\bar{E}_{n}\left(a^{-} b\right)\right)\left(\pi_{n^{*}}\left(\left\{z \mid f(z \mid m)=g\left(\left\langle z(m+k) \mid k<m^{\prime}\right\rangle\right)\right\}\right)\right)=1\right. \\
& \Leftrightarrow\left(E_{n}\left(\pi_{n}(a) \pi_{n}(b)\right)\right)\left(\left\{z \mid\left(\pi_{n^{*}}(f)\right)(z \mid m)=\left(\pi_{n^{*}}(g)\right)\left(\left\langle z(m+k) \mid k<m^{\prime}\right\rangle\right)\right\}\right)=1 \\
& \Leftrightarrow \llbracket \pi_{n^{*}}(f) \rrbracket_{\pi_{n}(a), E_{n}}^{M_{n^{*}}}=\llbracket \pi_{n^{*}}(g) \rrbracket_{\pi_{n}(b), E_{n}}^{M_{n^{*}}} .
\end{aligned}
$$

A similar argument shows that $\pi_{n+1}: \bar{M}_{n+1} \rightarrow M_{n+1}$ is elementary, as required by $(\mathrm{c})$.

To check that the rest of (c) - that $C_{n+1} \subseteq \operatorname{range}\left(\pi_{n+1}\right)$-holds, let $d \in C_{n+1}$. By property (iii) of $C_{n+1}$, let $a \in C_{n}$ and $f \in C_{n^{*}}$ be such that $d=\mathbb{L} f \mathbb{1}_{a, E_{n}}^{M_{n^{*}}}$. By (c) and (d) for $n$ and $n^{*}$ and by property (iv) of $C_{n}$ and $C_{n^{*}}$, let $\pi_{n}(\bar{a})=a$ and $\pi_{n^{*}}(\bar{f})=f$. By the elementarity of $\pi_{n}, \quad \bar{a} \in{ }^{<\omega}\left(\operatorname{support}\left(\bar{E}_{n}\right)\right)$. The elementarity of $\pi_{n^{*}}$ gives that $\bar{f}:{ }^{\ln (\bar{a})}\left(\operatorname{crit}\left(\bar{E}_{n}\right)\right) \rightarrow \bar{M}_{n^{*}}$, since $\pi_{n^{*}}\left(\operatorname{crit}\left(\bar{E}_{n}\right)\right)=$ $\pi_{n}\left(\operatorname{crit}\left(\bar{E}_{n}\right)\right)$ by $(\mathrm{j})$ and the fact that $\operatorname{crit}\left(\bar{E}_{n}\right) \leq \bar{\rho}_{n^{*}}$. Let then $\bar{d}=\llbracket \bar{f} \prod_{\bar{a}, \bar{E}_{n}}^{\bar{M}_{n^{*}}}$. $\pi_{n+1}(\bar{d})=\llbracket \pi_{n^{*}}(\bar{f}) \prod_{\pi_{n}(\bar{a}), E_{n}}^{M_{n^{*}}}=\llbracket f \prod_{a, E_{n}}^{M_{n^{*}}}=d$. Hence $d \in \operatorname{range}\left(\pi_{n+1}\right)$.

Let us next check (h). Let $d \in \bar{M}_{n^{*}} . \quad i_{\bar{E}_{n}}^{\bar{M}_{n^{*}}}(d)=\llbracket c_{d} \rrbracket_{\varnothing, \bar{E}_{n}}^{\bar{M}_{n^{*}}}$, where $c_{d}$ : ${ }^{0} V_{\text {crit }\left(\bar{E}_{n}\right)} \rightarrow M_{n^{*}}$ is the function with value $d$. Hence

$$
\pi_{n+1}\left(i_{\bar{E}_{n}}^{\bar{M}_{n^{*}}}(d)\right)=\llbracket c_{\pi_{n^{*}}(d)} \rrbracket_{\varnothing, E_{n}}^{M_{n^{*}}}=i_{E_{n}}^{M_{n^{*}}}\left(\pi_{n^{*}}(d)\right) .
$$

To check (i), note first that $\operatorname{Ult}\left(\bar{M}_{n}, \bar{E}_{n}\right)$ is wellfounded by Lemma 1.6. Since $\bar{\rho}_{n}<i \overline{\bar{E}}_{n}\left(\operatorname{crit}\left(\bar{E}_{n}\right)\right) \leq i \overline{\bar{M}}_{n}\left(\bar{\rho}_{n^{*}}\right)$ and $V_{\bar{\rho}_{n^{*}+1}} \cap M_{n^{*}}=V_{\bar{\rho}_{n^{*}+1}} \cap M_{n}$, Lemma $1.7\left(\right.$ a) gives that $V_{\bar{\rho}_{n}+1} \cap \operatorname{Ult}\left(\bar{M}_{n^{*}}, \bar{E}_{n}\right)=V_{\bar{\rho}_{n}+1} \cap \operatorname{Ult}\left(\bar{M}_{n}, \bar{E}_{n}\right)$. Since $\operatorname{support}\left(\bar{E}_{n}\right) \supseteq V_{\bar{\rho}_{n}+1} \cap \bar{M}_{n}$, it follows that

$$
V_{\rho_{n}+1} \cap \bar{M}_{n+1}=V_{\rho_{n}+1} \cap \operatorname{Ult}\left(\bar{M}_{n^{*}} ; \bar{E}_{n}\right)=V_{\bar{\rho}_{n}+1} \cap \bar{M}_{n} .
$$


The rest of the requirement of (i) will be seen to be fulfilled if we can prove that $n^{\prime} \leq n \Rightarrow \bar{\rho}_{n^{\prime}} \leq \bar{\rho}_{n}$. But $(\mathrm{j})$ and (c) give that $\pi_{n}\left(\bar{\rho}_{n^{\prime}}\right)=\pi_{n^{\prime}}\left(\bar{\rho}_{n^{\prime}}\right)=\rho_{n^{\prime}} \leq$ $\rho_{n}=\pi_{n}\left(\bar{\rho}_{n}\right)$. So the elementarity of $\pi_{n}$ implies that $\bar{\rho}_{n^{\prime}} \leq \bar{\rho}_{n}$.

Finally let us verify $(\mathrm{j})$. By what has just been proved, it is enough to show that $\pi_{n+1} \uparrow\left(V_{\bar{\rho}_{n}+1} \cap \bar{M}_{n+1}\right)=\pi_{n} \uparrow\left(V_{\bar{\rho}_{n}+1} \cap \bar{M}_{n}\right)$. Suppose then that $d \in V_{\bar{\rho}_{n+1}} \cap \bar{M}_{n+1}$. Since $\bar{\rho}_{n}+1<i \overline{\bar{E}}_{n^{*}}\left(\bar{\rho}_{n^{*}}\right), d=\llbracket f \prod_{a, \bar{E}_{n}}^{\bar{M}_{n^{*}}}$ for some $a$ and some $f:{ }^{\operatorname{lh}(a)}\left(V_{\operatorname{crit}\left(\bar{E}_{n}\right)}\right) \rightarrow V_{\bar{\rho}_{n^{*}}} \cdot \operatorname{crit}\left(\bar{E}_{n}\right) \leq \bar{\rho}_{n^{*}}$, so $f$ is essentially a member of $V_{\bar{\rho}_{n^{*}+1}}$. By Lemma 1.7(c), $d=\llbracket f \mathbb{1}_{a, \bar{E}_{n}}^{\bar{M}_{n}}$. Hence $\pi_{n}(d)=$ $\llbracket \pi_{n}(f) \rrbracket_{\pi_{n}(a), E_{n}}^{M_{n}}$. Since $\pi_{n}(f)$ is essentially a member of $V_{\rho_{n^{*}+1}}$, Lemma $1.7(\mathrm{c})$ gives that $\pi_{n}(d)=\llbracket \pi_{n}(f) \rrbracket_{\pi_{n}(a), E_{n}}^{M_{n^{*}}}$. But $\pi_{n}(f)=\pi_{n^{*}}(f)$, by $(\mathrm{j})$. Hence $\pi_{n}(d)=\llbracket \pi_{n^{*}}(f) \rrbracket_{\pi_{n}(a), E_{n}}^{M_{n^{*}}}$. But this is just $\pi_{n+1}\left(\llbracket f \rrbracket_{a, \bar{E}_{n}}^{\bar{M}_{n^{*}}}\right)=\pi_{n+1}(d)$.

Now let us turn to the case $n+1 \notin b$. We proceed in two steps, the first analogous to the case $n+1 \in b$, and the second to take care of $(\mathrm{k})$.

Define $\bar{E}_{n}$ and $\bar{\rho}_{n}$ as before by $\pi_{n}\left(\bar{E}_{n}\right)=E_{n}$ and $\pi_{n}\left(\bar{\rho}_{n}\right)=\rho_{n}$. As before, (e) and (f) hold. Now set $M_{n+1}^{\prime}=\operatorname{Ult}\left(\bar{M}_{n^{*}} ; \bar{E}_{n}\right) . M_{n+1}^{\prime}$ is wellfounded and countably closed by Lemmas 1.6 and 1.8 , and we have the elementary embedding $i \bar{E}_{n^{*}}: \bar{M}_{n^{*}} \rightarrow M_{n+1}^{\prime}$. In particular we get (b $\left.{ }^{\prime}\right) M_{n+1}^{\prime}$ is a countably closed set model of $\mathrm{ZC}+\Sigma_{2}$-replacement if $n^{*} \notin b$ and a countably closed proper class model of ZFC if $n^{*} \in b$.

Define $\pi_{n+1}^{\prime}: M_{n+1}^{\prime} \rightarrow M_{n+1}$ by

$$
\pi_{n+1}^{\prime}\left(\llbracket f \mathbb{1}_{a, \bar{E}_{n}}^{\bar{M}_{n^{*}}}\right)=\llbracket \pi_{n^{*}}(f) \mathbb{1}_{\pi_{n}(a), E_{n}}^{M_{n^{*}}} .
$$

The arguments of the case $n+1 \in b$ give us that $\pi_{n+1}^{\prime}$ is well defined and also $\left(\mathrm{d}^{\prime}\right) \pi_{n+1}^{\prime}: M_{n+1}^{\prime} \rightarrow V_{\gamma_{n+1}} \cap M_{n+1}$ is an elementary embedding with $C_{n+1} \subseteq$ range $\left(\pi_{n+1}^{\prime}\right)$, where $\gamma_{n+1}=i_{E_{n}}^{M n^{*}}\left(\xi_{n^{*}}\right)$ if $n^{*} \notin b$ and $\gamma_{n+1}=\mathrm{ON}$ if $n^{*}$ $\in b$

(i') $V_{\bar{\rho}_{n}+1} \cap M_{n+1}^{\prime}=V_{\bar{\rho}_{n}+1} \cap \bar{M}_{n}$;

$\left(\mathrm{j}^{\prime}\right) \pi_{n+1}^{\prime} \uparrow\left(V_{\bar{\rho}_{n}+1} \cap M_{n+1}^{\prime \prime}\right)=\pi_{n} \uparrow\left(V_{\bar{\rho}_{n}+1} \cap \bar{M}_{n}\right)$.

Let $C_{n+1}^{\prime}$ be the preimage of $C_{n+1}$ under $\pi_{n+1}^{\prime}$. Since $M_{n+1}^{\prime}$ is countably closed, $C_{n+1}^{\prime} \in M_{n+1}^{\prime}$ and $M_{n+1}^{\prime}$ satisfies " $C_{n+1}^{\prime}$ is countable."

Let $\pi_{n+1}^{\prime}\left(\xi_{n+1}^{\prime}\right)=\xi_{n+1} . M_{n+1}^{\prime}$ satisfies ZC and so $M_{n+1}^{\prime}$ contains a set $S$ of Skolem functions for $V_{\xi^{\prime}{ }_{n+1}} \cap M_{n+1}^{\prime}$.

Let $Z_{n+1}$ be the smallest subset of $V_{\xi^{\prime}{ }_{n+1}} \cap M_{n+1}^{\prime}$ such that

(i) $Z_{n+1}$ is countably closed;

(ii) $Z_{n+1}$ is closed under the Skolem functions in $S$;

(iii) $\left(V_{\bar{\rho}_{n}+1} \cap M_{n+1}^{\prime}\right) \cup C_{n+1}^{\prime}-\left\{\xi_{n+1}^{\prime}\right\} \subseteq Z_{n+1}$.

$Z_{n+1} \in M_{n+1}^{\prime}$ and $\left|Z_{n+1}\right|^{M_{n+1}^{\prime}}=\left|V_{\rho_{n}+1}\right|^{M_{n+1}^{\prime}}$.

Let $\bar{\pi}_{n+1}: \bar{M}_{n+1} \simeq Z_{n+1}$ with $\bar{M}_{n+1}$ transitive. Let $\pi_{n+1}=\pi_{n+1}^{\prime} \circ \bar{\pi}_{n+1}$. 
(b) holds. (d) holds since $\pi_{n+1}^{\prime} \uparrow\left(V_{\xi^{\prime}{ }_{n+1}} \cap M_{n+1}^{\prime}\right): V_{\xi^{\prime}{ }_{n+1}} \cap M_{n+1}^{\prime} \rightarrow V_{\xi_{n+1}} \cap M_{n+1}$ is an elementary embedding and since $C_{n+1}^{\prime}-\left\{\xi_{n+1}\right\} \in \operatorname{range}\left(\bar{\pi}_{n+1}\right)$. (i) holds since $V_{\bar{\rho}_{n}+1} \cap M_{n+1}^{\prime} \subseteq Z_{n+1}$ and $\left(\mathrm{i}^{\prime}\right)$ holds. (j) holds since $\bar{\pi}_{n+1} \uparrow\left(V_{\bar{\rho}_{n}+1} \cap \bar{M}_{n+1}\right)$ is the identity and $\left(\mathrm{j}^{\prime}\right)$ holds.

It remains only to check (k). $\operatorname{crit}\left(\bar{E}_{n}\right)$ is inaccessible in $\bar{M}_{n}$, so it is inaccessible in $\bar{M}_{n^{*}}$, since, by the same argument as in the case $n+1 \in b, \operatorname{crit}\left(\bar{E}_{n}\right) \leq \bar{\rho}_{n^{*}}$ and so $V_{\text {crit }\left(\bar{E}_{n}\right)+1} \cap \bar{M}_{n^{*}}=V_{\text {crit }\left(\bar{E}_{n}\right)+1} \cap \bar{M}_{n}$. Thus $i \bar{M}_{n_{n}^{*}}\left(\operatorname{crit}\left(\bar{E}_{n}\right)\right)$ is inaccessible in $M_{n+1}^{\prime} \cdot\left|Z_{n+1}\right|^{M_{n+1}^{\prime}}=\left|V_{\bar{\rho}_{n+1}}\right|^{M_{n+1}^{\prime}}<i \overline{\bar{E}}_{\bar{E}_{n}{ }^{*}}\left(\operatorname{crit}\left(\bar{E}_{n}\right)\right)$, because $\bar{\rho}_{n}+1 \subseteq$ $\operatorname{support}\left(\bar{E}_{n}\right) \subseteq V_{\left.i_{\bar{E}_{n}} \overline{\bar{N}}_{n}^{*}\left(\operatorname{crit}\left(\bar{E}_{n}\right)\right)\right)}$. Hence $\bar{M}_{n+1}$ belongs to $V_{i_{\bar{E}_{n}} \overline{\bar{M}}_{n}^{*}\left(\operatorname{crit}\left(\bar{E}_{n}\right)\right)} \cap M_{n+1}^{\prime}$. But

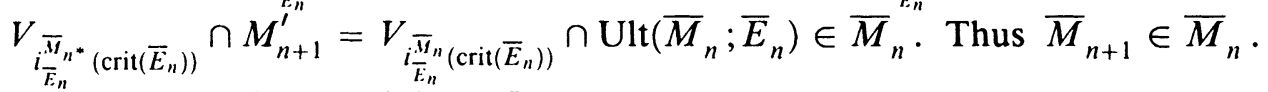
Since $\bar{M}_{n}=\bar{M}_{n+1}$ or $\bar{M}_{n} \in \bar{M}_{\hat{n}}=\bar{M}_{n+1}$, it follows that $\bar{M}_{n+1} \in \bar{M}_{\widehat{n+1}}$, as required.

Now let us use our construction to get a contradiction. For each $n, \bar{E}_{n} \in \bar{M}_{n}$ and so $\bar{E}_{n} \in \bar{M}_{\hat{n}}$ whenever $n \notin b$. If $n+1 \in b$, then either $n^{*}=n$ or else $n^{*}=\hat{n}$. In either case, $n+1 \in b$ implies that $\bar{E}_{n} \in \bar{M}_{n^{*}}$. Thus

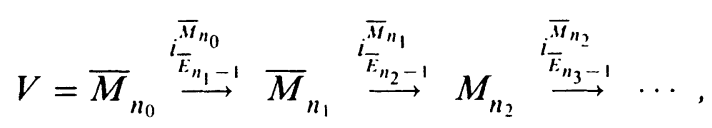

is a internal iteration, where $0=n_{0}, n_{1}, n_{2}, \ldots$ is an enumeration of $b$ in order of magnitude. Let $\bar{i}_{n_{k}, n_{k^{\prime}}}: \bar{M}_{n_{k}} \rightarrow \bar{M}_{n_{k^{\prime}}}$ be the obvious elementary embeddings for $k \leq k^{\prime}$. Let $\left(\bar{M}_{b},\left\langle\bar{i}_{n_{k}, n_{k^{\prime}}}, \mid k \leq k^{\prime} \in \omega\right\rangle\right)$ be the direct limit of the system $\left(\left\langle\bar{M}_{n_{k}} \mid k \in \omega\right\rangle,\left\langle\bar{i}_{n_{k}, n_{k^{\prime}}}, \mid k \leq k^{\prime} \in \omega\right\rangle\right)$. By Lemma $1.3, \bar{M}_{b}$ is wellfounded.

By (h) we have the commutative diagram

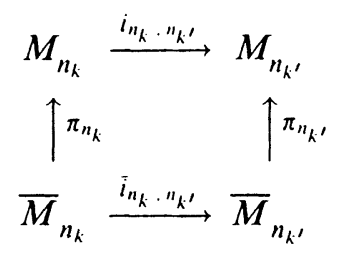

for $k \leq k^{\prime}$.

Let $\bar{\xi}_{n_{k}}$ be given by $\pi_{n_{k}}\left(\bar{\xi}_{n_{k}}\right)=\xi_{n_{k}}$, using property (ii) of $C_{n_{k}}$ and (c). Since $\xi_{n_{k^{\prime}}}<i_{n_{k}, n_{k^{\prime}}}\left(\xi_{n_{k}}\right)$, it follows that $\bar{\xi}_{n_{k^{\prime}}}<\bar{i}_{n_{k}, n_{k^{\prime}}}\left(\bar{\xi}_{n_{k}}\right)$. Thus $\left\langle\bar{i}_{n_{k}, b}\left(\bar{\xi}_{n_{k}}\right) \mid k \in \omega\right\rangle$ is an infinite descending sequence in the ordinals of $\bar{M}_{b}$. This contradiction completes the proof of the lemma.

Remark. We are dealing with countably closed iteration trees simply because the proofs of Lemmas 3.1 and 3.2 are simpler than the proofs of the assertions that would result from deleting all occurrences of "countably closed." Nevertheless 
those resulting assertions are true-and are proved in [11]. Armed with them, we could delete all occurrences of "countably closed" from the rest of this paper.

In the proof of the Main Theorem, we shall be concerned with iteration trees living in a certain $V_{\delta}$ and with homogeneous trees whose measures are $\delta^{+}$-complete. It will be important to know that the canonical elementary embeddings associated with the iteration trees act trivially on the elementary embeddings from the $\delta^{+}$-complete measures and that the latter act trivially on the former. This is the content of the following lemma. The proof of the lemma is at bottom the same as the proof that embeddings witnessing two distinct cardinals are measurable act trivially on one another. The extra complexity is due to the facts that our iteration tree embeddings are from extenders rather than measures and-more importantly-the extenders do not belong to the model the embeddings are applied to.

Lemma 3.3. Let $\mathscr{T}=\left(\prec,\left\langle M_{k} \mid k<\alpha\right\rangle,\left\langle E_{k} \mid k+1<\alpha\right\rangle,\left\langle\rho_{k} \mid k+1<\alpha\right\rangle\right)$ be an iteration tree on $V$ of length $\leq \omega$. Let $i_{m, n}$, for $m \preceq n$, be the canonical elementary embeddings associated with $\mathscr{T}$. Let $\mu$ be a $\delta$-complete measure on $X$, let $\nu$ be a $\delta$-complete measure on $X^{\prime}$, and let $q: X \rightarrow X^{\prime}$ be such that $\nu(A)=1 \Leftrightarrow \mu(\{z \mid q(z) \in A\})=1$, with $\delta$ a strong limit cardinal with each $\operatorname{support}\left(E_{k}\right) \in V_{\delta}$. Let $j=i_{\mu}$, let $j^{\prime}=i_{\nu}$, and let $j^{*}: \operatorname{Ult}(V ; \nu) \rightarrow$ $\operatorname{Ult}(V ; \mu)$ be the elementary embedding given by $j^{*}\left(\llbracket F \rrbracket_{\nu}\right)=\llbracket \check{F} \rrbracket_{\mu}$, where $\check{F}(z)=F(q(z))$. Then

(a) $i_{0, n}(j)=j \uparrow M_{n} ; i_{0, n}\left(j^{\prime}\right)=j^{\prime} \uparrow M_{n}$; and $i_{0, n}\left(j^{*}\right)=j^{*} \uparrow j^{\prime}\left(M_{n}\right)$.

(b) $\left.\left(j\left(i_{m, n}\right)\right) \uparrow \mathrm{ON}=\left(j^{\prime}\left(i_{m, n}\right)\right) \uparrow \mathrm{ON}\right)=i_{m, n} \uparrow \mathrm{ON}$.

Here, for example, $i_{0, n}(j)=\bigcup_{\alpha} i_{0, n}\left(j \uparrow V_{\alpha}\right)$.

Proof. We shall first prove (a). Let us begin by showing

(i) if $Y \subseteq i_{0, n}(X)$ and $Y \in M_{n}$, then $\left(i_{0, n}(\mu)\right)(Y)=1$ if and only if $\mu\left(\left\{z \mid i_{o, n}(z) \in Y\right\}\right)=1$.

Proof of (i). Assume (i) holds for all $n \leq m$. Let $Y \subset i_{0, m+1}(X)$ with $Y \in$ $M_{m+1}$. Assume that $\left(i_{0, m+1}(\mu)\right)(Y)=1$. Let $Y=\llbracket f \mathbb{1}_{a, E_{m}}^{M_{m^{*}}}$. Let

$$
K=\left\{x \in^{\ell h(a)}\left(V_{\operatorname{crit}\left(E_{m}\right)}\right) \mid\left(i_{0, m^{*}}(\mu)\right)(f(x))=1\right\} .
$$

$\left(E_{n}(a)\right)(K)=1$. For each $x \in K$, our inductive assumption gives that (i) holds for $n=m^{*}$, and so there is a $Z_{x} \subseteq X$ such that $\mu\left(Z_{x}\right)=1$ and $\left(\forall w \in Z_{x}\right)\left(i_{0, m^{*}}(w) \subseteq f(x)\right)$. Let $Z=\bigcap_{x \in K} Z_{x}$. Since $\mu$ is $\delta$-complete and $\delta>\operatorname{crit}\left(E_{m}\right), \mu(Z)=1$.

$$
\begin{aligned}
w \in Z & \Rightarrow(\forall x \in K)\left(w \in Z_{x}\right) \\
& \Rightarrow(\forall x \in K)\left(\left(i_{0, m^{*}}(w)\right) \in f(x)\right) \\
& \Rightarrow\left(E_{m}(a)\right)\left(\left\{x \mid i_{0, m^{*}}(w) \in f(x)\right\}\right)=1 \\
& \Rightarrow i_{m^{*}, m+1}\left(i_{0, m^{*}}(w)\right) \in \mathbb{I} f \mathbb{1}_{a, E_{n}}^{M_{m^{*}}} \Rightarrow i_{0, m+1}(w) \in Y .
\end{aligned}
$$


We have shown then that $\left(i_{0, n+1}(\mu)\right)(Y)=1 \Rightarrow \mu\left(\left\{z \mid i_{0, n+1}(z) \in Y\right\}\right)=1$. If $\left(i_{0, n+1}(\mu)\right)(Y)=0$, then $\mu\left(\left\{z \mid i_{0, n+1}(z) \in i_{0, n+1}(X)-Y\right\}\right)=1$ and so $\mu\left(\left\{z \mid i_{0, m+1}(z) \in Y\right\}\right)=0$.

For each $n \in \omega$ and each $F \in M_{n} \cap^{\left(i_{0 . n}(X)\right)} M_{n}$, let $\Phi_{n}(F): X \rightarrow M_{n}$ be given by

$$
\left(\Phi_{n}(F)\right)(z)=F\left(i_{0, n}(z)\right) .
$$

(ii) For each $G \in{ }^{X} M_{n}$ there is an $F \in M_{n} \cap^{\left(i_{0, n}(X)\right)} M_{n}$ such that

$$
\llbracket \Phi_{n}(F) \rrbracket_{\mu}=\llbracket G \rrbracket_{\mu}
$$

Proof of (ii). Assume that (ii) holds for all $n \leq m$. Let $G: X \rightarrow M_{m+1}$. For each $z \in X$, let $G(z)=\llbracket f_{z} \rrbracket_{a_{z}, E_{m}}^{M_{m^{*}}}$. Since $\mu$ is $\delta$-complete and $\delta>$ $\left|\operatorname{support}\left(E_{m}\right)\right|$ there must be an $a \in{ }^{<\omega}\left(\operatorname{support}\left(E_{m}\right)\right)$ such that $\mu\left(\left\{z \mid a_{z}=a\right\}\right)=1$. For this $a, \mu\left(\left\{z \mid G(z)=\llbracket f_{z} \rrbracket_{a, E_{m}}^{M_{m^{*}}}\right\}\right)=1$. Define $G^{*}: X \rightarrow M_{m^{*}}$ by

$$
G^{*}(z)=f_{z} .
$$

Our inductive assumption gives that (ii) holds for $n=m^{*}$, and so there is an $F^{*} \in M_{m^{*}}$ with $F^{*}: i_{0, m^{*}}(X) \rightarrow M_{m^{*}}$ such that $\llbracket \Phi_{m^{*}}\left(F^{*}\right) \rrbracket_{\mu}=\llbracket G^{*} \rrbracket_{\mu}$. Define $h:{ }^{\ell h(a)}\left(V_{\operatorname{crit}\left(E_{m}\right)}\right) \rightarrow M_{m^{*}}$ by letting $h(x): i_{0, m^{*}}(X) \rightarrow M_{m^{*}}$ with $(h(x))(z)=\left(F^{*}(z)\right)(x)$. Let $F=\llbracket h \rrbracket_{a, E_{m}}^{M_{m^{*}}}$. We must show that

$$
\mu\left(\left\{z \mid F\left(i_{0, m+1}(z)\right)=G(z)\right\}\right)=1 .
$$

We know that $\mu\left(\left\{z \mid G(z)=\llbracket f_{z} \mathbb{I}_{a, E_{n}}^{M_{m^{*}}} \& F^{*}\left(i_{0, m^{*}}(z)\right)=G^{*}(z)\right\}\right)=1$. Let $z$ belong to this set. Then

$$
(h(x))\left(i_{0, m^{*}}(z)\right)=\left(F^{*}\left(i_{0, m^{*}}(z)\right)\right)(x)=\left(G^{*}(z)\right)(x)=f_{z}(x) .
$$

Hence $F\left(i_{0, m+1}(z)\right)=F\left(i_{m^{*}, m+1}\left(i_{0, m^{*}}(z)\right)\right)=\llbracket f_{z} \mathbb{1}_{a, E_{m}}^{M_{m^{*}}}=\llbracket f_{z} \rrbracket_{a_{z}, E_{m}}^{M_{m^{*}}}=G(z)$.

(i) implies that $\Phi_{n}$ induces an elementary embedding of $\left(i_{0, n}(j)\right)\left(M_{n}\right)$ into $j\left(M_{n}\right)$. To see this, let $\psi\left(v_{1}, \ldots, v_{k}\right)$ be a formula of set theory and let $G_{1}, \ldots, G_{k}$ be elements of $M_{n} \cap^{i_{0, n}(X)} M_{n}$.

$$
\begin{aligned}
& \left(i_{0, n}(j)\right)\left(M_{n}\right) \vDash \psi\left[\llbracket G_{1} \rrbracket_{i_{0, n}(\mu)}^{M_{n}}, \ldots, \llbracket G_{k} \rrbracket_{i_{0, n}(\mu)}^{M_{n}}\right] \\
& \Leftrightarrow\left(i_{0, n}(\mu)\right)\left(\left\{z \mid M_{n} \vDash \psi\left[G_{1}(z), \ldots, G_{l}(z)\right]\right\}\right)=1 \\
& \Leftrightarrow\left(\text { by (i)) } \mu \left(\left\{z \mid M_{n} \vDash \psi\left[G_{1}\left(i_{0, n}(z), \ldots, G_{k}\left(i_{0, n}(z)\right)\right]\right\}\right)=1\right.\right. \\
& \Leftrightarrow \mu\left(\left\{z \mid M_{n} \vDash \psi\left[\left(\Phi_{n}\left(G_{1}\right)\right)(z), \ldots,\left(\Phi_{n}\left(G_{k}\right)\right)(z)\right]\right\}\right)=1 \\
& \Leftrightarrow j\left(M_{n}\right) \vDash \psi\left[\llbracket \Phi_{n}\left(G_{1}\right) \mathbb{1}_{\mu}, \ldots, V \llbracket \llbracket \Phi_{n}\left(G_{k}\right) \rrbracket_{\mu}^{V}\right] \text {. }
\end{aligned}
$$

(ii) implies that the elementary embedding induced by $\Phi_{n}$ is a surjection and so is an isomorphism, i.e. is the identity. Since $\Phi_{n}$ sends each constant function to the constant function with the same value, it follows that $\left(i_{0 . n}(j)\right)=j \uparrow M_{n}$. 
Using functions $\Phi_{n}^{\prime}$ analogous to the $\Phi_{n}$, we similarly get that $\left(i_{0, n}\left(j^{\prime}\right)\right)=j^{\prime}$ । $M_{n}$. To see that $\left(i_{0, n}\left(j^{*}\right)\right)=j^{*} \mid j^{\prime}\left(M_{n}\right)$, note first that $\left(i_{0, n}\left(j^{*}\right)\right)\left(\llbracket F \mathbb{\prod}_{i_{0, n}(\nu)}^{M_{n}}\right)=$ $\llbracket F \circ i_{0, n}(q) \mathbb{1}_{i_{0, n}, l_{n}(\mu)}^{M_{n}}$, but $j^{*}\left(\llbracket \Phi_{n}^{\prime}(F) \rrbracket_{\nu}^{V}\right)=\llbracket\left(\Phi_{n}^{\prime}(F)\right) \circ q \mathbb{\rrbracket}_{\mu}^{V}=\llbracket F \circ i_{0, n} \circ q \mathbb{\rrbracket}_{\mu}^{V}=$ $\llbracket F \circ i_{0, n}(q) \circ i_{0, n} \rrbracket_{\mu}^{V}=\llbracket \Phi_{n}\left(F \circ i_{0, n}(q)\right) \rrbracket_{\mu}^{V}$. Since $\Phi_{n}$ and $\Phi_{n}^{\prime}$ induce the identities on equivalence classes, this completes the proof of (a).

Now let us turn to (b). Let $n$ be arbitrary. Since $\mu$ is $\delta$-complete, $j(V)$ is closed under sequences of length $<\delta$. The elementarity of $i_{0, n^{*}}$ thus implies that $\left(i_{0, n^{*}}(j)\right)\left(M_{n^{*}}\right)$ has the same sequences of ordinals of length $<i_{0, n^{*}}(\delta)$ as does $M_{n^{*}}$. Now $\left(i_{0, n^{*}}(j)\right)\left(M_{n^{*}}\right)=j\left(M_{n^{*}}\right)$, as we already showed. Hence $j\left(M_{n^{*}}\right)$ has the same sequences of ordinals of length $<i_{0, n^{*}}(\delta)$, and hence the same functions $F:{ }^{<\omega}\left(V_{\operatorname{crit}\left(E_{n}\right)}\right) \rightarrow \mathrm{ON}$ as does $M_{n^{*}}$. Since $j\left(E_{n}\right)=E_{n}$, it follows that $j\left(i_{n^{*}, n+1}\right)=i_{n^{*}, n+1}$. Since $n$ was arbitrary, (b) follows by the definition of the $i_{m, n}$.

Remark. In our applications, $\mu$ and $\nu$ will be, for example, $\mu_{x \mid n}$ and $\mu_{x \mid m}$, respectively, for some $m<n \in \omega$, where $\left\langle\mu_{s} \mid s \in{ }^{<\omega} \omega\right\rangle$ witnesses that some tree is $\delta$-homogeneous. In this case $q$ is given by $q(t)=t \uparrow m$.

In $\S 5$ we shall often be dealing with iteration trees of a particularly simple sort: alternating chains. Let $\prec^{*}$ be the partial ordering of $\omega$ given by

$$
m \prec{ }^{*} n \Leftrightarrow(m<n \&(m=0 \vee n-m \text { is even })) .
$$

An alternating chain on $V$ of length $\alpha \leq \omega$ is an iteration tree on $V$ of length $\alpha$ whose tree ordering is $\prec^{*} \mid \alpha$. We shall usually describe alternating chains as systems $\left(\left\langle M_{n} \mid n<\alpha\right\rangle,\left\langle E_{n} \mid n+1<\alpha\right\rangle,\left\langle\rho_{n} \mid n+1<\alpha\right\rangle\right)$, i.e. we omit " $\prec^{*}$." Pictorially an alternating chain is

$$
\begin{array}{r}
V=M_{0} \stackrel{\stackrel{i_{E_{0}}^{M_{0}}}{\longrightarrow}}{\longrightarrow} M_{1} \stackrel{\stackrel{i_{E_{2}}^{M_{1}}}{\longrightarrow}}{\longrightarrow} M_{3} \stackrel{\stackrel{i_{E_{4}}^{M_{3}}}{\longrightarrow}}{\longrightarrow} \cdots \\
\searrow_{E_{E_{1}} M_{0}}^{N_{0}} \\
M_{2} \stackrel{i_{E_{3}}^{M_{2}}}{\longrightarrow} M_{4} \stackrel{i_{E_{5}}^{M_{4}}}{\longrightarrow} \cdots
\end{array}
$$

\section{REFLECTING CARDINALS}

In this section we develop the theory of Woodin cardinals in order to get the technical tools we need to build iteration trees in $\S 5$.

First, however, we give some proofs which are not needed for $\S 5$ but show where Woodin cardinals sit in the hierarchy of large cardinal axioms. A cardinal $\kappa$ is $\lambda$-supercompact if there is an elementary $j: V \rightarrow M$ with $M$ transitive, $\operatorname{crit}(j)=\kappa$, and ${ }^{\lambda} M \subseteq M . \kappa$ is superstrong if there is an elementary $j$ : $V \rightarrow M$ with $M$ transitive, $\operatorname{crit}(j)=\kappa$, and $V_{j(\kappa)} \subseteq M . \kappa$ is Shelah if for every $f: \kappa \rightarrow \kappa$ there is a $j: V \rightarrow M$ with $M$ transitive, $\operatorname{crit}(j)=\kappa$, and $V_{(j(f))(\kappa)} \subseteq M$. A cardinal $\delta$ is Woodin if for every $f: \delta \rightarrow \delta$ there is a $\kappa<\delta$ closed under $f$ and there is a $j: V \rightarrow M$ with $M$ transitive, $\operatorname{crit}(j)=\kappa$, and 
$V_{(j(f))(\kappa)} \subseteq M . \kappa$ is $\lambda$-strong if there is an elementary $j: V \rightarrow M$ with $M$ transitive, $\operatorname{crit}(j)=\kappa$, and $V_{\lambda} \subseteq M$.

The concept of $\lambda$-supercompactness is due to $\mathrm{R}$. Solovay. The notions of $\lambda$-strong and superstrong cardinals arose out of the work of W. Mitchell. Shelah and Woodin isolated the notions named after them. The lemma that follows catalogues some basic facts about the relative strengths of the large cardinal axioms corresponding to these concepts. No part of the lemma is due to us.

Lemma 4.1. (1) If $\kappa$ is $2^{\kappa}$-supercompact then there are $\kappa$ superstrong cardinals $<\kappa$. (2) If $\kappa$ is superstrong then $\kappa$ is Shelah and there are $\kappa$ Shelah cardinals $<\kappa$. (3) If $\kappa$ is Shelah then $\kappa$ is Woodin and there are $\kappa$ Woodin cardinals $<\kappa$. (4) If $\delta$ is Woodin then $\delta$ is inaccessible and there are $\delta$ cardinals $\kappa<\delta$ such that $(\forall \lambda<\delta) \kappa$ is $\lambda$-strong.

Proof. (1) Let $j: V \rightarrow M$ witness that $\kappa$ is $2^{\kappa}$-supercompact. Let $E$ be the extender derived from $j$ with support $V_{j^{(\kappa)}} \cap M$. For each $a \in \epsilon^{<\omega}\left(V_{j^{(\kappa)}}\right) \cap M$, $E(a)$ is a measure on ${ }^{\ln (a)}\left(V_{\kappa}\right)$ and $(E(a))(X)=1 \Leftrightarrow a \in j(X)$. Thus $E$ depends only on $V_{j^{(\kappa)}} \cap M$ and $j \uparrow V_{\kappa+1}$. But $j \uparrow V_{\kappa+1} \in{ }^{\left(V_{\kappa+1}\right)} M$ so $j \uparrow V_{\kappa+1} \in$ $\ddot{M}$. Hence $E \in M . i_{E}^{M}: M \rightarrow N$ is an element embedding, belongs to $M$, and is such that $V_{j(k)} \cap M \in N$. We have the commutative diagram

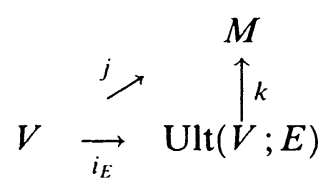

Hence $j(\kappa)=k\left(i_{E}(\kappa)\right) \geq i_{E}(\kappa) \geq i_{E}^{M}(\kappa)$. It follows that $V_{i_{i}^{M}(\kappa)} \cap M \in N$. Thus $i_{E}^{M}$ witnesses that $\kappa$ is superstrong in $M$. To see that (1) follows from this, suppose $\beta<\kappa$. In $M$ there is a superstrong cardinal between $\beta=j(\beta)$ and $j(\kappa)$, so in $V$ there is a superstrong cardinal between $\beta$ and $\kappa$.

(2) Let $j: V \rightarrow M$ witness that $\kappa$ is superstrong. $j$ also witnesses that $\kappa$ is Shelah, for if $f: \kappa \rightarrow \kappa$ then $V_{(j(f))(\kappa)} \subseteq V_{j(\kappa)} \subseteq M$. Let us check that $\kappa$ is Shelah in $M$ also, from which (2) follows. Suppose $f: \kappa \rightarrow \kappa$. Let $E$ be the extender derived from $j$ with support $V_{(j(f))(\kappa)+1} \cup V_{\kappa+1} . E \in V_{j(\kappa)}$ so $E \in M$. We have $k: \operatorname{Ult}(V ; E) \rightarrow M$ with $k \circ i_{E}=j$ and with $k$ i $V_{(j(f))(\kappa)+1}=$ the identity. Thus $k\left(i_{E}(f)\right)=j(f) . k \uparrow V_{\kappa+1}$ is the identity, so $k(\kappa)=\kappa$. It follows that $k\left(\left(i_{E}(f)\right)(\kappa)\right)=k\left(i_{E}(f)\right)(k(\kappa))=(j(f))(\kappa)$. Hence $i_{E}(f)(\kappa) \leq($ actually $=)(j(f))(\kappa)$, and so $V_{\left(i_{E}(f)\right)(\kappa)} \subseteq V_{(j(f))(\kappa)} \subseteq \operatorname{Ult}(V ; E)$. Since $V_{j(\kappa)} \cap \operatorname{Ult}(V ; E)=V_{j(\kappa)} \cap \operatorname{Ult}(M ; E)$ and $i_{E}^{V}(f)=i_{E}^{M}(f)$, we have that $V_{\left(i_{E}^{M}(f)\right)(\kappa)} \subseteq \operatorname{Ult}(M ; E)$. This means that $i_{E}^{M}$ witnesses that $\kappa$ is Shelah in $M$.

(3) Let $\kappa$ be Shelah and let $f: \kappa \rightarrow \kappa$. Let $g(\alpha)=\alpha+2+f(\alpha)+1$. Let $j: V \rightarrow M$ witness that $\kappa$ is Shelah, with respect to $g$. Let $E$ be the extender derived from $j$ with support $V_{(j(f))(\kappa)} \cdot E:{ }^{<\omega}\left(V_{(j(f))(\kappa)}\right) \rightarrow \mathscr{P}(\mathscr{P}(\kappa))$, so 
$E$ is coded by an element of $V_{\kappa+2+(j(f))(\kappa)+1}$. Hence $E \in M .(j(f))(\kappa)=$ $\left(i_{E}(f)\right)(\kappa)=\left(i_{E}^{M}(f)\right)(\kappa)$. Furthermore $\kappa$ is closed under $j(f)$, since $j(f)$ । $\kappa=f$. Thus $i_{E}^{M}$ witnesses the Woodinness of $j(\kappa)$ with respect to $j(f)$ in $M$. By the elementarity of $j$, some embedding witnesses the Woodinness of $\kappa$ with respect to $f$ in $V$. Since $f$ was arbitrary, $\kappa$ is Woodin. Note also that $E \in V_{j(\kappa)} \cap M$, so we also get that some $i_{F}$ such that $F$ is an extender $\in V_{\kappa}$ witnesses the Woodinness of $\kappa$ with respect to $f$ in $V$. (We will see later that Woodin cardinals always have this property.) Such an extender belongs to $M$ and so witnesses the Woodinness of $\kappa$ with respect to $f$ in $M$. Moreover, for any elementary $k: V \rightarrow N$ with $N$ transitive and $\operatorname{crit}(k)=\kappa$, this same extender witnesses the Woodinness of $\kappa$ with respect to $f$ in $N$. But our argument has shown that for every $f$ and every such $N, \kappa$ is Woodin with respect to $f$ in $N$. Since there is such an $N$, the remaining part of (3) holds.

(4) Let $\delta$ be Woodin. If $\delta$ is not regular then there is a $\gamma<\delta$ and an $f: \gamma \rightarrow \delta$ with unbounded range. Let $g(0)=\gamma, g(\alpha)=f(1+\alpha)$ for $\alpha<\gamma$, and $g(\alpha)=0$ for $\delta>\alpha \geq \gamma$. No ordinal $>0$ is closed under $g$, so $g$ contradicts Woodinness. If limit ordinal $\gamma<\delta$ and $2^{\gamma} \geq \delta$, let $f: \delta \rightarrow \delta$ be such that $f(0)=\gamma$. If $j$ witnesses Woodinness of $\kappa$ with respect to $f$, then $\operatorname{crit}(j)$ is a measurable cardinal. But $\operatorname{crit}(j)>f(0)$ so $\operatorname{crit}(j)$ cannot even be a strong limit cardinal. This contradiction completes the proof that $\kappa$ is inaccessible.

Assume that $\beta<\delta$ is such that, for all $\kappa$ with $\beta \leq \kappa<\delta$, there is a $\lambda<\delta$ such that $\kappa$ is not $\lambda$-strong. For $\kappa \geq \beta$, let $\lambda(\kappa)$ be the least $\lambda \geq \kappa$ such that $\kappa$ is not $\lambda$-strong. Let $f(\kappa)=\beta$ for $\kappa<\beta$ and let $f(\kappa)=\lambda(\kappa)+3$ for $\kappa \geq \beta$. Let $j: V \rightarrow M$ witness Woodinness of $\delta$ with respect to $f$. Let $\kappa=\operatorname{crit}(j)$. Let $E$ be the extender derived from $j$ with support $V_{(j(\lambda))(\kappa)} . E \in M$. Since $V_{(j(\lambda))(\kappa)} \in \operatorname{Ult}(M ; E)$, it follows that $E$ witness that $\kappa$ is $((j(\lambda))(\kappa))$-strong in $M$. But $\kappa>\beta$, since $\kappa$ is closed under $f$, and so $\kappa>j(\beta)=\beta$. This gives us the contradiction that $\kappa$ is not $((j(\lambda))(\kappa))$-strong in $M$.

The argument just given generalizes to give the following useful consequence (actually an equivalent) of Woodinness.

Lemma 4.2. Let $\delta$ be Woodin and let $A \subseteq V_{\delta}$. There are arbitrarily large $\kappa<\delta$ such that for all $\alpha<\delta$ there is $a j: V \rightarrow M$ with $j$ elementary, $\operatorname{crit}(j)=\kappa$, $M$ transitive, $V_{\alpha} \subseteq M$, and $V_{\alpha} \cap A=V_{\alpha} \cap j(A)$. Moreover $j$ can be chosen as $i_{E}$ for $E$ an extender $\in V_{\delta}^{\alpha}$ with $\operatorname{support}(E) \supseteq V_{\alpha}$ and with $\operatorname{support}(E)$ countably closed.

Proof. Assume for a contradiction that $\beta<\delta$ is such that for all $\kappa$ with $\delta>\kappa \geq \beta$ there is an $\alpha<\delta$ for which there is no extender $E \in V_{\delta}$ with $\operatorname{support}(E) \supseteq V_{\alpha}, \operatorname{support}(E)$ countably closed, $\operatorname{crit}(E)=\kappa$ and $V_{\alpha} \cap A=$ $V_{\alpha} \cap i_{E}(A)$. For $\kappa \geq \beta$ let $\alpha(\kappa)$ be the least $\alpha \geq \kappa+2$ with this property. Let $f(\kappa)=\beta+1$ for $\kappa<\beta$ and let $f(\kappa)$ be $\alpha(\kappa)+\omega_{1}+1$ for $\kappa \geq \beta$. Let $j: V \rightarrow M$ witness Woodinness of $\delta$ with respect to $f$. Let $\kappa=\operatorname{crit}(j)$. Let $E$ be the extender derived from $j$ with support $V_{(j(\alpha))(\kappa)+\omega_{1}} \cdot E \in M$, 
$E \in V_{j(\kappa)}$, and $\operatorname{support}(E)$ is countably closed. We have the commutative diagram

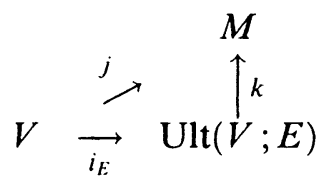

with $k \uparrow V_{(j(\alpha))(\kappa)+\omega_{1}}$ the identity. $k\left(i_{E}(A)\right)=j(A)$, so $j(A) \cap V_{(j(\alpha))(\kappa)}=$ $i_{E}(A) \cap V_{(j(\alpha))(\kappa)} \cdot i_{E} \uparrow V_{\kappa+1}=i_{E}^{M} \uparrow V_{\kappa+1}$, so $j(A) \cap V_{(j(\alpha))(\kappa)}=i_{E}^{M}(A) \cap$ $V_{(j(\alpha))(\kappa)}$. Since $j(A) \cap V_{\kappa}=A \cap V_{\kappa}, i_{E}^{M}(j(A)) \cap V_{i_{E}^{M}(\kappa)}=i_{E}^{M}(A) \cap V_{i_{E}^{M}(\kappa)}$, and so $i_{E}^{M}(j(A)) \cap V_{(j(\alpha))(\kappa)}=i_{E}^{M}(A) \cap V_{(j(\alpha))(\kappa)}=j(A) \cap V_{(j(\alpha))(\kappa)} . \kappa$ is closed under $f$, so $\kappa>\beta$ and therefore $\kappa>j(\beta)$. Thus $E$ contradicts the definition of the function $j(\alpha)$ in $M$.

Though we do not need the next lemma later, we prove it to show that Woodinness of $\delta$ is always witnessed by extenders in $V_{\delta}$.

Lemma 4.3. Let $\delta$ be Woodin and let $f: \delta \rightarrow \delta$. There is an extender $E \in$ $V_{\delta}$, with countably closed support and with $\operatorname{crit}(E)$ closed under $f$, such that $V_{\left(i_{E}(f)\right)(\operatorname{crit}(E))} \subseteq \operatorname{support}(E)$.

Proof. We may assume without loss of generality, that $f(\gamma) \geq \gamma$ for all $\gamma<\delta$. Let $A=f$, i.e. $A=\{\langle\gamma, f(\gamma)\rangle \mid \gamma<\delta\}$. Let $\kappa$ be as given by Lemma 4.2. Let $\alpha=f(\kappa)+3$. Let $E$ be given as in Lemma 4.2. Since $V_{f(\kappa)} \subseteq \operatorname{support}(E)$, it suffices to show that $f(\kappa)=\left(i_{E}(f)\right)(\kappa)$. But $\langle\kappa, f(\kappa)\rangle \in V_{f(\kappa)+3} \cap A$ and so $\langle\kappa, f(\kappa)\rangle \in V_{f(\kappa)+3} \cap i_{E}(A)$. Hence $\left(i_{E}(f)\right)(\kappa)=f(\kappa)$.

Let $\delta$ be a strong limit cardinal. Let $\omega \leq \alpha \leq \delta$ and $\beta$ be ordinals and let $z \in{ }^{<\omega}\left(V_{\delta+\beta}\right)$. The $(\alpha, \beta)$-type of $z$ relative to $\delta$ is the set of formulas $\varphi$ of the language of set theory with constants $c(a)$ for elements $a$ of $V_{\alpha}$ and with a constant $c(\delta)$ for $\delta$ if $\beta>0$ such that $\left(V_{\delta+\beta} ; \in,\left\langle a \mid a \in V_{\alpha}\right\rangle\right) \vDash \varphi[z]$ (or, more briefly, $\left.V_{\delta+\beta} \vDash \varphi[z]\right)$. Note for future reference that the $(\alpha, \beta)$-type of $z$ is essentially a subset of $V_{\alpha}$, for $\alpha$ infinite. It follows that if $\omega \leq \alpha^{\prime}<\alpha<\delta$ and $\beta^{\prime}<\beta$ then the $\left(\alpha^{\prime}, \beta^{\prime}\right)$-type of $z$ relative to $\delta$ is expressed by a single element of the $(\alpha, \beta)$-type of $z^{-}\left\langle\beta^{\prime}\right\rangle$.

For strong limit cardinals $\delta$, ordinals $\beta$, and $z \in \epsilon^{<\omega}\left(V_{\delta+\beta}\right)$, we say that $\kappa<$ $\delta$ is $\beta$-reflecting in $z$ relative to $\delta$ if for all $\alpha<\delta$ there is an extender $E \in V_{\delta}$ with $\operatorname{support}(E)$ countably closed, $\operatorname{crit}(E)=\kappa, i_{E}(\delta)=\delta, V_{\alpha} \subseteq \operatorname{support}(E)$, and the $(\alpha, \beta)$-type of $z$ relative to $\delta$ in $V$ the same as the $\left(\alpha, i_{E}(\beta)\right)$-type of $i_{E}(z)$ relative to $\delta$ in $\operatorname{Ult}(V ; E)$. Note for future reference that if $\kappa<\delta$ and $\beta^{\prime}<\beta$, then the assertion that $\kappa$ is $\beta^{\prime}$-reflecting in $z$ relative to $\delta$ is expressed by a single element of the $(\kappa+1, \beta)$-type of $z^{-}\left\langle\beta^{\prime}\right\rangle$. The only nonobvious point in the verification of this is using the fact that $V_{\delta+i_{E}\left(\beta^{\prime}\right)} \cap \operatorname{Ult}(V ; E)$ satisfies $\varphi\left[i_{E}(z), a\right]$ if and only if $E(a)\left(\left\{x \mid V_{\delta+\beta^{\prime}}\right.\right.$ satisfies $\left.\left.\varphi[z, x]\right\}\right)=1$. 
Lemma 4.4. Let $\delta$ be an inaccessible. The following are equivalent.

(a) $\delta$ is Woodin.

(b) For all $\beta$ and all $z \in{ }^{<\omega}\left(V_{\delta+\beta}\right)$, the set of $\kappa$ which are $\beta$-reflecting in $z$ relative to $\delta$ is unbounded in $\delta$.

(c) For all $z \in{ }^{<\omega}\left(V_{\delta+1}\right)$, there is a $\kappa<\delta$ such that $\kappa$ is 1-reflecting in $z$ relative to $\delta$.

Proof. We first show that (a) implies (b). Assume that $\delta$ is Woodin, that $\beta$ is an ordinal, and that $z \in^{<\omega}\left(V_{\delta+\beta}\right)$. In the sequel we omit the phrase "relative to $\delta$." Let $A=$ the $(\delta, \beta)$-type of $z$. By Lemma 4.2 there are unboundedly many $\kappa<\delta$ such that for all $\alpha<\delta$ there is an extender $E \in V_{\delta}$ with $\operatorname{crit}(E)=\kappa$, $V_{\alpha} \subseteq \operatorname{support}(E), \operatorname{support}(E)$ countably closed, and $V_{\alpha} \cap A=V_{\alpha} \cap i_{E}(A) . \delta$ is inaccessible and $E \in V_{\delta}$, so $i_{E}(\delta)=\delta . i_{E}(A)=$ the $\left(\delta, i_{E}(\beta)\right)$-type of $i_{E}(z)$ in $\operatorname{Ult}(V ; E)$, so the fact that $V_{\alpha} \cap A=V_{\alpha} \cap i_{E}(A)$ means that the $(\alpha, \beta)$-type of $z$ in $V$ is the same as the $\left(\alpha, i_{E}(\beta)\right)$-type of $i_{E}(z)$ in $\operatorname{Ult}(V ; E)$. Thus all of these unboundedly many $\kappa$ are $\beta$-reflecting in $z$.

We complete the proof by showing that (c) implies (a). Assume that $\delta$ satisfies (c). Once again we shall omit "relative to $\delta$." Let $f: \delta \rightarrow \delta$. Let $\kappa$ be 1 -reflecting in $\langle f\rangle$. Let $\alpha=\max \{\kappa+1, \sup \{f(\xi)+1 \mid \xi \leq \kappa\}\}$. Let $E$ witness for $\alpha$ that $\kappa$ is 1 -reflecting in $\langle f\rangle$. Let $\xi \leq \kappa$ and let $\gamma=f(\xi)$. The fact that $\gamma=f(\xi)$ is expressed by a member of the $(\alpha, 1)$-type of $\langle f\rangle$. Hence the same element of the $(\alpha, 1)$-type of $i_{E}(\langle f\rangle)$ expresses the fact that $\left(i_{E}(f)\right)(\xi)=\gamma$. For $\xi<\kappa$ this gives us that $\left(i_{E}(f)\right)(\xi)=\gamma=f(\xi)<\alpha \leq i_{E}(\kappa)$ and so that $f(\xi)<\kappa$. Hence $\kappa$ is closed under $f$. For $\xi=\kappa$ we get that $\left(i_{E}(f)\right)(\kappa)=\gamma=f(\kappa)$. Since $V_{f(\kappa)} \subseteq \operatorname{support}(E), i_{E}$ witnesses the Woodinness of $\kappa$ with respect to $f$.

Our goal in the rest of this section is to prove a technical lemma which will give us the individual steps in our construction of iteration trees in $\S 5$. First we prove a preliminary lemma.

Lemma 4.5. Let $M$ and $N$ be countably closed, transitive, proper class models of ZFC. Let $\delta$ be inaccessible. Let $\kappa<\delta$ with $V_{\kappa+1} \cap M=V_{\kappa+1} \cap N$. Let $\beta$ and $\beta^{\prime}$ be ordinals and let $x \in{ }^{<\omega}\left(V_{\delta+\beta}\right) \cap M$ and $x^{\prime} \in{ }^{<\omega}\left(V_{\delta+\beta^{\prime}}\right) \cap N$. Suppose that the $(\kappa, \beta)$-type of $x$ relative to $\delta$ in $M$ is the same as the $\left(\kappa, \beta^{\prime}\right)$-type of $x^{\prime}$ relative to $\delta$ in $N$. Suppose that $E$ witnesses, for some $\alpha<\delta$, that $\kappa$ is $\beta$-reflecting in $x$ relative to $\delta$ in $M$. Then Ult $(N ; E)$ is wellfounded, $V_{\alpha} \cap \operatorname{Ult}(N ; E)=V_{\alpha} \cap M$, and the $\left(\alpha, i_{E}^{N}\left(\beta^{\prime}\right)\right)$-type of $i_{E}^{N}\left(x^{\prime}\right)$ relative to $\delta$ in $\operatorname{Ult}(N ; E)$ is the same as the $(\alpha, \beta)$-type of $x$ relative to $\delta$ in $M$.

Proof. We omit "relative to $\delta$." By Lemma 1.6, Ult $(N ; E)$ is wellfounded. Since $V_{\kappa+1} \cap N=V_{\kappa+1} \cap M$ we have that $V_{i_{i}^{N}(\kappa)+1} \cap \operatorname{Ult}(N ; E)=V_{i_{E}^{M}(\kappa)+1} \cap$ $\operatorname{Ult}(M ; E)$. Since $\alpha \leq i_{E}^{M}(\kappa)$, it follows that $V_{\alpha} \cap \operatorname{Ult}(N ; E)=V_{\alpha} \cap \operatorname{Ult}(M ; E)$ $=V_{\alpha} \cap M$. 
Let $\tau=$ the $(\alpha, \beta)$-type of $x$ in $M=$ the $\left(\alpha, i_{E}^{M}(\beta)\right)$-type of $i_{E}^{M}(x)$ in $\operatorname{Ult}(M ; E)$. Let $\tau=\llbracket f f \mathbb{1}_{a}^{M}$. Since $f:{ }^{\ell h(a)}\left(V_{\kappa}\right) \rightarrow V_{\kappa+1}$, it follows that $\tau=$ $\llbracket f \mathbb{1}_{a}^{N}$. Furthermore, the values of $f$ are $(\xi, \beta)$-types of $x$ in $M$ for various $\xi \leq \kappa$. By assumption, the $(\xi, \beta)$-type of $x$ in $M$ is the same as the $\left(\xi, \beta^{\prime}\right)$ -

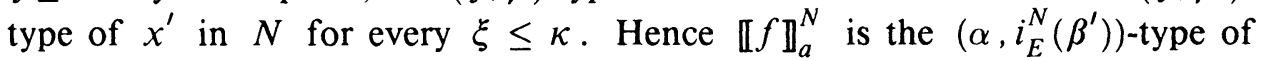
$i_{E}^{N}\left(x^{\prime}\right)$ in $\operatorname{Ult}(N ; E)$.

Lemma 4.6 (One-Step Lemma). Let $M$ and $N$ be countably closed, transitive, proper class models of ZFC. Let $\delta$ be Woodin in $M$ and inaccessible in $V$, let $\kappa<\delta$, let $\eta<\delta$, let $\beta$ and $\beta^{\prime}$ be ordinals, let $\xi<\beta$, let $x$ and $y$ belong to ${ }^{<\omega}\left(V_{\delta+\xi}\right) \cap M$, let $x^{\prime} \in{ }^{<\omega}\left(V_{\delta+\beta^{\prime}}\right) \cap N$, and let $\varphi(v)$ be a formula of set theory. Assume

(a) $V_{\kappa+1} \cap M=V_{\kappa+1} \cap N$;

(b) the $(\kappa, \beta)$-type of $x$ relative to $\delta$ in $M$ is the same as the $\left(\kappa, \beta^{\prime}\right)$-type of $x^{\prime}$ relative to $\delta$ in $N$;

(c) $\kappa$ is $\beta$-reflecting in $x$ relative to $\delta$ in $M$;

(d) $V_{\delta+\beta} \cap M \vDash \varphi(\xi)$.

Then there is an $E \in V_{\delta} \cap M$ such that $M$ satisfies " $E$ is an extender with countably closed support," such that $\operatorname{crit}(E)=\kappa$, and such that $\operatorname{Ult}(N ; E)$ is wellfounded, and there are also $\kappa^{*}$ with $\eta<\kappa^{*}<\delta, \xi^{*}<i_{E}^{N}\left(\beta^{\prime}\right)$ with $i_{E}\left(x^{\prime}\right) \in{ }^{<\omega}\left(V_{\delta+\xi^{*}}\right)$, and $y^{*} \in{ }^{<\omega}\left(V_{\delta+\xi^{*}}\right) \cap \operatorname{Ult}(N ; E)$, with the properties that $\operatorname{support}(E) \supseteq V_{\kappa^{*}+1} \cap M$ and

$\left(\mathrm{a}^{*}\right) V_{\kappa^{*}+1} \cap \operatorname{Ult}(N ; E)=V_{\kappa^{*}+1} \cap M$;

$\left(\mathrm{b}^{*}\right)$ the $\left(\kappa^{*}, \xi^{*}\right)$-type of $i_{E}^{N}\left(x^{\prime}\right)^{-} y^{*}$ relative to $\delta$ in $\mathrm{Ult}(N ; E)$ is the same as the $\left(\kappa^{*}, \xi\right)$-type of $x^{\frown} y$ relative to $\delta$ in $M$;

$\left(\mathrm{c}^{*}\right) \kappa^{*}$ is $\xi^{*}$-reflecting in $i_{E}^{N}\left(x^{\prime}\right)^{-} y^{*}$ relative to $\delta$ in $\operatorname{Ult}(N ; E)$;

$\left(\mathrm{d}^{*}\right) V_{\delta+i_{E}^{N}\left(\boldsymbol{\beta}^{\prime}\right)} \vDash \varphi\left[\xi^{*}\right]$;

$\left(\mathrm{e}^{*}\right)$ if $y$ is a finite sequence of ordinals, then $y^{*}$ is definable in $V_{\delta+i_{E}^{N}\left(\beta^{\prime}\right)} \cap$ $\operatorname{Ult}(N ; E)$ from $\delta, i_{N}^{E}\left(x^{\prime}\right)$, and elements of $V_{\kappa^{*}+1} \cap \operatorname{Ult}(N ; E)$.

Proof. Once more we omit "relative to $\delta$." Let $\kappa^{*}$ be such that $\eta<\kappa^{*}<\delta$ and $\kappa^{*}$ is $\xi$-reflecting in $x^{-} y$ in $M$. Let $E$ witness that $\kappa$ is $\beta$-reflecting in $x$ in $M$ for $\kappa^{*}+1$. By Lemma 4.5 we have $\left(\mathrm{a}^{*}\right)$ and that the $\left(\kappa^{*}+1, i_{E}^{N}\left(\beta^{\prime}\right)\right)$-type of $i_{E}^{N}\left(x^{\prime}\right)$ in $\operatorname{Ult}(N, E)$, which is wellfounded, is the same as the $\left(\kappa^{*}+1, \beta\right)$-type of $x$ in $M$.

Let $\tau$ be the $\left(\kappa^{*}, \xi\right)$-type of $x^{\frown} y$ in $M . V_{\delta+\beta} \cap M$ satisfies

$(\exists u)(\exists v)\left(v\right.$ is an ordinal \& $u \epsilon^{<\omega}\left(V_{\delta+v}\right)$

$\& \tau=$ the $\left(\kappa^{*}, v\right)$-type of $x^{-} u$ relative to $\delta$

$\& \kappa^{*}$ is $v$-reflecting in $x^{-} u$ relative to $\left.\delta \& \varphi(v)\right)$. 
Therefore (the formal version of) this formula belongs to the $\left(\kappa^{*}+1, \beta\right)$ type of $x \in M$. Hence the formula belongs to the $\left(\kappa^{*}+1, i_{E}^{N}\left(\beta^{\prime}\right)\right)$-type of $i_{E}^{N}\left(x^{\prime}\right)$ in $\operatorname{Ult}(N ; E)$. So there are $y^{*}$ and $\xi^{*}$ such that $\xi^{*}$ is an ordinal and $\tau=$ the $\left(\kappa^{*}, \xi^{*}\right)$-type of $i_{E}^{N}\left(x^{\prime}\right)^{-} y^{*}$ in Ult $(N ; E)$ and $\kappa^{*}$ is $\xi^{*}$-reflecting in $i_{E}^{N}\left(x^{\prime}\right)^{-} y^{*}$ in $\operatorname{Ult}(N ; E)$ and $V_{\delta+i_{E}^{N}\left(\beta^{\prime}\right)} \cap \operatorname{Ult}(N ; E) \vDash \varphi\left[\xi^{*}\right]$. This gives $\left(\mathrm{b}^{*}\right),\left(\mathrm{c}^{*}\right)$, and $\left(\mathrm{d}^{*}\right)$. If $y$ is a finite sequence of ordinals, then so is $y^{*}$ and we may take the lexicographically least $y^{* \frown}\left\langle\xi^{*}\right\rangle$. This $y^{*}$ is definable from $\delta, \tau, \kappa^{*}$, and $i_{E}^{N}\left(x^{\prime}\right)$ in $V_{\delta+i_{E}^{N}\left(\beta^{\prime}\right)} \cap \operatorname{Ult}(N ; E)$. Since $\tau$ essentially belongs to $V_{\kappa^{*}+1},\left(\mathrm{e}^{*}\right)$ holds.

Remark. The hypotheses of the One-Step Lemma actually imply that $\delta$ is Woodin in $N$ and so in $\operatorname{Ult}(N ; E)$. We see this as follows. Since $\xi<\beta$, $\beta>0$ and so, by hypothesis (b), $V_{\delta+\beta} \cap M$ and hence $V_{\delta+\beta^{\prime}} \cap N$ satisfy " $\delta$ is Woodin." (In the applications we shall always know without this argument that $\delta$ is Woodin in $N$.)

\section{The Main Theorem}

Thoroughout this section let $\delta$ be a fixed inaccessible cardinal. When we speak of $(\alpha, \beta)$-types and of $\beta$-reflecting cardinals we shall omit the expression "relative to $\delta . "$

We begin by illustrating the use of the One-Step Lemma in building iteration trees by proving the following theorem (which will not directly be used later).

Theorem 5.1. If $\delta$ is Woodin then for every $n \in \omega$ there is a countably closed alternating chain on $V$ of length $n+1$.

Proof. The theorem is trivially true for $n=0$. Assume then that $n>0$. Let $\kappa_{0}$ be $(n-1)$-reflecting in $\varnothing$. Assume inductively that we have, for some $k$ with $0 \leq k<n$, a countably closed alternating chain $\left(\left\langle M_{m} \mid m \leq k\right\rangle,\left\langle E_{m}\right|\right.$ $\left.m<k\rangle,\left\langle\rho_{m} \mid m<k\right\rangle\right)$ on $V$ and ordinals $\kappa_{0}, \kappa, \ldots, \kappa_{k}$ with each $E_{m} \in V_{\delta}$, with $\operatorname{crit}\left(E_{m}\right)=\kappa_{m}$ for $m<k$, and with $\kappa_{k} \leq \rho_{k-1}$ if $k>0$. Assume inductively also that the $\left(\kappa_{k}, n-k-1\right)$-type of $\varnothing$ in $M_{k}$ is the same as the $\left(\kappa_{k}, n-k-1\right)$-type of $\varnothing$ in $M_{k-1}$, (where $k \dot{-j}=k-j$ if $k \geq j$ and $k \dot{-j}=0$ if $k \leq j)$ and that $\kappa_{k}$ is $(n-k-1)$-reflecting in $\varnothing$ in $M_{k}$.

If $n-k>1$, apply the One-Step Lemma with $M=M_{k}, N=M_{k-1}$, $\kappa=\kappa_{k}, \eta=\kappa_{k}, \beta=\beta^{\prime}=n-k-1, \xi=n-k-2, \varphi(v)$ as " $\delta+v$ is the greatest ordinal," and $x=x^{\prime}=y=\varnothing$. Let $E, \kappa^{*}, \xi^{*}$, and $y^{*}$ be as given by the One-Step Lemma. Clearly $\xi^{*}=n-k-2$ and $y^{*}=\varnothing$. Let $E_{k}=E$ and $\rho_{k}=\kappa^{*}$. Let $M_{k+1}=\operatorname{Ult}\left(M_{k-1} ; E_{k}\right)$. Let $\kappa_{k+1}=\kappa^{*}$. Our inductive assumptions hold for $k+1$.

If $n=k+1$ let $E_{k}$ witness that $\kappa_{k}$ is 0-reflecting in $\varnothing$ in $M_{k}$ with $\alpha=\kappa_{k}$, let $\rho_{k}=\kappa_{k}$, and let $M_{n}=\operatorname{Ult}\left(M_{k-1} ; E_{k}\right)$.

The theorem just proved makes no use of the fact that Lemma 4.4 gives arbitrarily large reflecting cardinals $<\delta$, and it similarly makes no real use of 
the $\eta$ of the One-Step Lemma. To see how we can make use of these ingredients, consider the following game $\mathscr{G}_{n}$ :

$$
\begin{array}{cccccccccc}
I & \alpha_{0} & & \alpha_{1} & & & \cdots & \alpha_{n-1} & & \\
I I & & \kappa_{0} & & \left\langle E_{0}, \rho_{0}, \kappa_{1}\right\rangle & & & \left\langle E_{n-2}, \rho_{n-2}, \kappa_{n-1}\right\rangle & & \left\langle E_{n-1}, \rho_{n-1}\right\rangle
\end{array}
$$

The first player to disobey one of the following rules loses a play of $\mathscr{G}_{n}$. If all the rules are obeyed then $I I$ wins.

(1) $\alpha_{k}<\delta$.

(2) $\alpha_{k}<\kappa_{k}<\delta, \kappa_{k+1} \leq \rho_{k}, \rho_{n-1}>\alpha_{n}, E_{k} \in V_{\delta}$, the $E_{k}$ and the $\rho_{k}$ generate a countably closed alternating chain on $V$, and $\operatorname{crit}\left(E_{k}\right)=\kappa_{k}$.

Theorem 5.2. If $\delta$ is Woodin, then for every $n \in \omega$ player II has a winning strategy for $\mathscr{G}_{n}$.

The proof of Theorem 5.2 is just like that of Theorem 5.1, except that we take $\kappa_{0}>\alpha_{0}$, in the application of the One-Step Lemma we take $\eta=\alpha_{k+1}$, and in the case $n=k+1$ we let $E_{k}$ witness that $\kappa_{k}$ is 0 -reflecting in $\varnothing$ in $M_{k}$ with $\alpha=\alpha_{n}+1$.

In fact Theorem 5.2 does not require full Woodinness of $\delta$. It suffices that $\delta$ be Woodin with respect to functions definable in $V_{\delta}$. Indeed this property can be shown equivalent with $I I$ 's having winning strategies for all the $\mathscr{G}_{n}$.

A further ingredient of Lemma 4.4 and of the One-Step Lemma is missing from Theorem 5.2 as well as from Theorem 5.1. In the proofs of these theorems we took the $z$ of Lemma 4.4 and the $x, x^{\prime}$, and $y$ of the One-Step Lemma to be the empty sequence. In the construction to come we shall be building an alternating chain and-in the two branches of the alternating chain-be building branches through the images of a certain tree $T$. The parameters just mentioned will allow us to extend one of these branches when we are given an extension of the other.

Superficially it appears that the One-Step Lemma is useful only for building finite alternating chains. The requirement that $\xi<\beta$ seems to militate against building infinite chains via the lemma. Since we need to build infinite alternating chains, we must sidestep this problem. There is more than one way to do so. Here we proceed as follows. and

For the rest of this section let $\lambda<c_{0}<c_{1}<c_{2}$ be cardinals such that $\lambda>\delta$

(i) All $c_{k}, k \leq 2$, are strong limit cardinals of cofinality greater than $\delta$;

(ii) $c_{0}$ and $c_{1}$ satisfy the same formulas in $V_{c_{2}}$, allowing parameters from $V_{\lambda+1}$ (i.e., the $\left(\lambda+1, c_{2}\right)$-type of $c_{0}$ is the same as the $\left(\lambda+1, c_{2}\right)$-type of $c_{1}$ ).

Lemma 5.3. For any $\lambda>\delta$, there exist $c_{0}, c_{1}$, and $c_{2}$ satisfying (i) and (ii) with $\lambda<c_{0}<c_{1}<c_{2}$.

Proof. Let $Z$ be the class of all strong limit cardinals of cofinality $>\delta$. Let $c_{2}$ be the $\left|V_{\lambda+2}\right|^{+}$th element of $Z$. There are only $\left|V_{\lambda+2}\right|$ possible $\left(\lambda+1, c_{2}\right)$-types, 
so there must be $c_{0}<c_{1}<c_{2}$ with $c_{0}$ and $c_{1} \in Z$ and $c_{0}$ and $c_{1}$ having the same $\left(\lambda+1, c_{2}\right)$-type.

Our plan is to use the "descending" sequence of ordinals:

$$
c_{0}+1, c_{0} \sim c_{1}, c_{0}+1, c_{0} \sim c_{1}, \ldots
$$

The key points are (a) If $\kappa<\delta$ is $c_{0}$-reflecting in some $z \in{ }^{<\omega}\left(V_{\lambda+1}\right)$, then $V_{c_{2}}$ satisfies " $\kappa$ is $c_{0}$-reflecting in $z$ " and so (ii) gives that $V_{c_{2}}$ satisfies " $\kappa$ is $c_{1}$-reflecting in $z$." (b) For $\alpha<\delta$ and $z \in^{<\omega}\left(V_{\lambda+1}\right)$, the $\left(\alpha, c_{0}\right)$-type of $z$ is the same as the $\left(\alpha, c_{1}\right)$-type of $z$. Condition (i) is for notational convenience. It guarantees that $c_{0}, c_{0}$, and $c_{2}$ will be fixed by all the embeddings arising in iteration trees whose extenders belong to $V_{\delta}$.

We first consider the problem of embedding normal form for $\neg p[T]$, where $T$ is a $\delta^{+}$-homogeneous tree, and of the homogeneity of the corresponding $T^{*}$.

For $\kappa_{0}<\delta$ and $T$ a tree on $\omega \times \lambda$ (the $\lambda$ we chose above), consider the following game $\mathscr{G}_{\kappa_{0}}^{T}$.

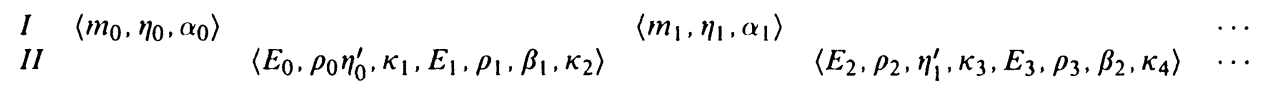

The first player to disobey one of the following rules loses a play of $\mathscr{G}_{\kappa_{0}}^{T}$. If all rules are obeyed, $I I$ wins.

R1. The $E_{n}$ and $\rho_{n}$ generate a countably closed alternating chain on $V$ with all $E_{n} \in V_{\delta}$.

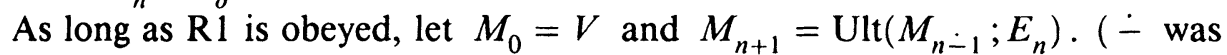
defined during the proof of Theorem 5.1.) Also let $i_{m, n}$, for $m \leq n$ and $m=0$ or $n-m$ even, be the canonical elementary embeddings associated with the alternating chain.

R2. $\kappa_{n}=\operatorname{crit}\left(E_{n}\right)$.

R3. $\kappa_{2 n}<\alpha_{n}<\delta$.

R4. $\kappa_{2 n+2}>\kappa_{2 n+1}>\alpha_{n}$.

R5. $\beta_{n+1}<i_{2 n, 2 n+2}\left(\beta_{n}\right)$, where $\beta_{0}=c_{0}$.

R6. Let $t_{n}=\left\langle i_{2 k, 2 n-2}\left(\eta_{k}\right) \mid k<n\right\rangle . \quad\left\langle\left\langle m_{k} \mid k \leq n\right\rangle, t_{n+1}\right\rangle \in i_{0,2 n}(T)$.

R7. Let $u_{n}=\left\langle i_{2 k+1,2 n-1}\left(\eta_{k}^{\prime}\right) \mid k<n\right\rangle .\left\langle\left\langle m_{k} \mid k \leq n\right\rangle, u_{n+1}\right\rangle \in i_{0,2 n+1}(T)$.

R8. $\eta_{n}^{\prime}$ is definable in $V_{c_{2}} \cap M_{2 n+1}$ from elements of $\left(V_{\kappa_{2 n+1}+1} \cap M_{2 n+1}\right) \cup$ $\left\{\delta, i_{0,2 n+1}(T), c_{0}\right\}$.

Remark. A few words about what is happening in the game are perhaps in order. $I$ is playing a $\langle x, f\rangle \in[T]$, along with some ordinals $\alpha_{i} . I I$ is playing an alternating chain, an $f^{\prime}$ such that $\left\langle x, f^{\prime}\right\rangle$ belongs to the image of $[T]$ in the direct limit along the odd branch of the alternating chain, and a sequence of ordinals $\beta_{i}$ which give a descending chain in the direct limit along the even branch. The sequence $f^{\prime}$ is required to belong to a certain subset of the image of $[T(x)]$, a subset of size less than $\delta$. The role of the $\alpha_{i}$ is to keep the critical points of the extenders increasing. 
Lemma 5.4. If $\delta$ is Woodin and $T$ is a tree on $\omega \times \lambda$, then the set of $\kappa_{0}<\delta$ such that II has a winning strategy for $\mathscr{G}_{\kappa_{0}}^{T}$ is unbounded in $\delta$.

Proof. By Lemma 4.5, there are unboundedly many $\kappa<\delta$ such that $\kappa$ is $\left(c_{0}+1\right)$-reflecting in $\langle T\rangle$. Let $\kappa_{0}$ be any such $\kappa$. We shall construct a winning strategy $\tau$ for II for $\mathscr{G}_{\kappa_{0}}^{T}$.

Assume inductively that we have defined $\tau$ on all positions of length $<2 n$. Suppose that we are given a position of length $2 n$ which is consistent with $\tau$ and is such that neither player has disobeyed the rules. Let us also assume inductively

$(*)_{n} \quad \kappa_{2 n} \leq \rho_{2 n-1}$ if $n>0$;

$(* *)_{n}$ the $\left(\kappa_{2 n}, \beta_{n}+1\right)$-type of $\left\langle i_{0,2 n}(T)\right\rangle^{-} i_{2 n-2,2 n}\left(t_{n}\right)$ in $M_{2 n}$ is the same as the $\left(\kappa_{2 n}, c_{0}+1\right)$-type of $\left\langle i_{0,2 n-1}(T)\right\rangle u_{n}$ in $M_{2 n-1}$;

$(* * *)_{n} \quad \kappa_{2 n}$ is $\left(\beta_{n}+1\right)$-reflecting in $\left\langle i_{0,2 n}(T)\right\rangle i_{2 n-2,2 n}\left(t_{n}\right)$ in $M_{2 n}$.

Note that $(* *)_{0}$ and $(* * *)_{0}$ hold. (Recall that $\beta_{0}=c_{0}$.)

Let $I$ play $\left\langle m_{n}, \eta_{n}, \alpha_{n}\right\rangle$ and assume that this move obeys the rules. Thus $\alpha_{n}<\delta$ and $t_{n+1}=i_{2 n-2,2 n}\left(t_{n}\right)^{-}\left\langle\eta_{n}\right\rangle$ is such that $\left\langle\left\langle m_{k} \mid k \leq n\right\rangle, t_{n+1}\right\rangle \in$ $i_{0,2 n}(T)$.

The assumptions of the One-Step Lemma hold with $M=M_{2 n}, N=M_{2 n-1}$, $\kappa=\kappa_{2 n}, \eta=\alpha_{n}, \beta=\beta_{n}+1, \beta^{\prime}=c_{0}+1, \xi=\beta_{n}, x=\left\langle i_{0,2 n}(T)\right\rangle i_{2 n-2,2 n}\left(t_{n}\right)$, $x^{\prime}=\left\langle i_{0,2 n-1}(T)\right\rangle u_{n}, y=\left\langle\eta_{n}\right\rangle$, and $\varphi(v)$ as " $\delta+v$ is the largest ordinal." $(*)_{n}$ and the fact that R1 has been obeyed give (a) of the One-Step Lemma, $(* *)_{n}$ gives (b), and $(* * *)_{n}$ gives $(\mathrm{c})$. (d) is obvious.

Let $E, \kappa^{*}, \xi^{*}$, and $y^{*}$ be given by the One-Step Lemma. $\left(\mathrm{b}^{*}\right)$ of the OneStep Lemma implies that $y^{*}$ is a sequence of length one. $\left(\mathrm{d}^{*}\right)$ implies that $\xi^{*}=c_{0}$.

Let $\tau$ call for $I I$ to play

$$
E_{2 n}=E, \quad \rho_{2 n}=\kappa^{*}, \quad\left\langle\eta_{n}^{\prime}\right\rangle=y^{*}, \quad \text { and } \kappa_{2 n+1}=\kappa^{*} .
$$

(The rest of $I I$ 's move will be specified later.)

By $(*)_{n},\left(\mathrm{a}^{*}\right)$ of the One-Step Lemma, and Lemma $3.1, \mathrm{R} 1$ is obeyed. (We still have an alternating chain.) The definitions of $\kappa_{2 n+1}$ and $\rho_{2 n}$, together with $\left(b^{*}\right)$ and $\left(c^{*}\right)$ of the One-Step Lemma, give

$(*)_{n}^{\prime} \quad \kappa_{2 n+1} \leq \rho_{2 n} ;$

$(* *)_{n}^{\prime}$ the $\left(\kappa_{2 n+1}, c_{0}\right)$-type of $\left\langle i_{0,2 n+1}(T)\right\rangle^{-} u_{n+1}$ in $M_{2 n+1}$ is the same as the $\left(\kappa_{2 n+1}, \beta_{n}\right)$-type of $\left\langle i_{0,2 n}(T)\right\rangle^{-} t_{n+1}$ in $M_{2 n}$;

$(* * *)_{n}^{\prime} \quad \kappa_{2 n+1}$ is $c_{0}$-reflecting in $\left\langle i_{0,2 n+1}(T)\right\rangle^{-} u_{n+1}$ in $M_{2 n+1}$.

$\mathrm{R} 2$ is obeyed, since $\kappa_{2 n}=\kappa^{*}=\operatorname{crit}(E)=\operatorname{crit}\left(E_{2 n}\right) . \mathrm{R} 4$ is obeyed, since $\kappa_{2 n+1}=\kappa^{*}>\eta=\alpha_{n}$. By $(* *)_{n}^{\prime}$ and the fact that R6 has been obeyed,

$$
\left\langle\left\langle m_{k} \mid k \leq n\right\rangle, u_{n+1}\right\rangle \in i_{0,2 n+1}(T)
$$


and so $\mathrm{R} 7$ is obeyed. $\left(\mathrm{e}^{*}\right)$ of the One-Step Lemma gives that $y^{*}$ is definable in $V_{\delta+c_{0}+1} \cap M_{2 n+1}$ from $\delta,\left\langle i_{0,2 n+1}(T)\right\rangle^{\sim} i_{2 n-1,2 n+1}\left(u_{n}\right)$, and elements of $V_{\kappa_{2 n+1}+1} \cap M_{2 n+1}$. Since earlier moves obeyed R8, $i_{2 n-1,2 n+1}\left(u_{n}\right)$ is definable in $V_{c_{2}} \cap M_{2 n+1}$ from elements of $\left(V_{\kappa_{2 n+1}+1} \cap M_{2 n+1}\right) \cup\left\{\delta, i_{0,2 n+1}(T), c_{0}\right\}$. It follows that R8 is obeyed.

By property (ii) of $\left\langle c_{0}, c_{1}, c_{1}\right\rangle$ (we have been using property (i) without mention), $(* *)_{n}^{\prime}$ and $(* * *)_{n}^{\prime}$ imply

$(* *)_{n}^{\prime \prime}$ the $\left(\kappa_{2 n+1}, c_{1}\right)$-type of $\left\langle i_{0,2 n+1}(T)\right\rangle^{-} u_{n+1}$ in $M_{2 n+1}$ is the same as the $\left(\kappa_{2 n+1}, \beta_{n}\right)$-type of $\left\langle i_{0,2 n}(T)\right\rangle^{\frown} t_{n+1}$ in $M_{2 n}$;

$(* * *)_{n}^{\prime \prime} \quad \kappa_{2 n+1}$ is $c_{1}$-reflecting in $\left\langle i_{0,2 n+1}(T)\right\rangle^{-} u_{n+1}$ in $M_{2 n+1}$.

The assumptions of the One-Step Lemma hold with $M=M_{2 n+1}, N=M_{2 n}$, $\kappa=\kappa_{2 n+1}, \eta=\kappa_{2 n+1}, \beta=c_{1}, \beta^{\prime}=\beta_{n}, \xi=c_{0}+1, x=\left\langle i_{0,2 n+1}(T)\right\rangle^{-} u_{n+1}$, $x^{\prime}=\left\langle i_{0,2 n}(T)\right\rangle^{-} t_{n+1}, y=\varnothing$, and $\varphi(v)$ as the trivial formula $v=v .(*)_{n}^{\prime}$ and the fact that R1 has been obeyed give (a) of the One-Step Lemma, $(* *)^{\prime \prime}$ gives $(\mathrm{b})$, and $(* * *)^{\prime \prime}$ gives (c). (d) is trivial.

Let $E, \kappa^{*}, \xi^{*}$, and $y^{*}$ be given by the One-Step Lemma. ( $\left.{ }^{*}\right)$ of the One-Step Lemma implies that $y^{*}=\varnothing$. $\left(\mathrm{b}^{*}\right)$ of the One-Step Lemma implies that $\xi^{*}$ is a successor ordinal.

Let $\tau$ call for $I I$ to play $E_{2 n+1}=E, \rho_{2 n+1}=\kappa^{*}, \beta_{n+1}+1=\xi^{*}$, and $\kappa_{2 n+2}=\kappa^{*}$. By $(*)_{n}^{\prime},\left(\mathrm{a}^{*}\right)$ of the One-Step Lemma, and Lemma 3.1, R1 is obeyed. The definitions of $\kappa_{2 n+2}$ and $\rho_{2 n+1}$ and $\left(\mathrm{b}^{*}\right)$ and $\left(\mathrm{c}^{*}\right)$ of the OneStep Lemma give $(*)_{n+1},(* *)_{n+1}$, and $(* * *)_{n+1} . \mathrm{R} 2$ is obeyed, since $\kappa_{2 n+1}=$ $\kappa^{*}=\operatorname{crit}(E)=\operatorname{crit}\left(E_{2 n+1}\right)$. Since $\beta_{n+1}<\xi^{*}<i_{2 n, 2 n+2}\left(\beta^{\prime}\right)=i_{2 n, 2 n+2}\left(\beta_{n}\right), \mathrm{R} 5$ is obeyed. Thus all rules are obeyed.

Theorem 5.5. If $T$ is a $\delta^{+}$-homogeneous tree on $\omega \times \lambda$ and $\delta$ is Woodin, then $\neg p[T]$ has embedding normal form.

Proof. By Lemma 5.4 let $\kappa_{0}<\delta$ be such that $I I$ has a winning strategy $\tau$ for the game $\mathscr{G}_{\kappa_{0}}^{T}$. For each $\langle s, t\rangle \in T$, let $\left\langle M_{k}(s, t) \mid k \leq 2 \ell h(s)\right\rangle,\left\langle E_{k}(s, t)\right|$ $k<2 \ell h(s)\rangle,\left\langle\rho_{k}(s, t) \mid k<2 \ell h(s)\right\rangle,\left\langle u_{k}(s, t) \mid k \leq \ln (s)\right\rangle$, and $\left\langle\beta_{k}(s, t)\right|$ $k \leq \ell h(s)\rangle$ result from play consistent with $\tau$ in which $I$ plays $m_{k}=s(k)$, $\eta_{k}=\left(i_{0,2 k}(s, t)\right)(t(k))$, and $\alpha_{k}=\kappa_{2 k}($ already played by $I I)$ for $k<\ell h(s)$.

Let $\left\langle\mu_{s} \mid s \in{ }^{<\omega} \omega\right\rangle$ witness that $T$ is $\delta^{+}$-homogeneous. Since each $E_{k}(s, t) \in$ $V_{\delta}$, R8 implies that all $u_{k}(s, t)$ are definable in $V_{c_{2}}$ from elements of $V_{\delta} \cup$ $\left\{\delta, c_{0}, T\right\}$. Since the $\mu_{s}$ are $\delta^{+}$complete, it follows that the $E_{k}(s, t), \rho_{k}(s, t)$, and $u_{k}(s, t)$ are, for each $s$, constant on a set of measure 1 with respect to $\mu_{s}$ and, consequently, that the $M_{k}(s, t)$ are also constant. Let $M_{k}(s), E_{k}(s)$, $\rho_{k}(s)$, and $u_{k}(s)$ be these constant values, and let $\mu_{s}\left(X_{s}\right)=1$ with these constant values attained whenever $t \in X_{s}$. Note that $s_{1} \subseteq s_{2}$ implies that $M_{k}\left(s_{1}\right)=M_{k}\left(s_{2}\right)$ for $k \leq 2 \ell h\left(s_{1}\right), E_{k}\left(s_{1}\right)=E_{k}\left(s_{2}\right)$ and $\rho_{k}\left(s_{1}\right)=\rho_{k}\left(s_{2}\right)$ for $k<2 \ell h\left(s_{1}\right)$, and $u_{k}\left(s_{1}\right)=u_{k}\left(s_{2}\right)$ for $k \leq \ell h\left(s_{1}\right)$. Let $i_{k_{1}, k_{2}}(s)$, for 
$k_{2} \leq 2 \ell h(s), k_{1} \leq k_{2}$, and $k_{1}=0$ or else $k_{2}-k_{1}$ even, be the canonical elementary embeddings associated with

$$
\left(\left\langle M_{k}(s) \mid k \leq 2 \ell h(s)\right\rangle,\left\langle E_{k}(s) \mid k<2 \ell h(s)\right\rangle,\left\langle\rho_{k}(s) \mid k<2 \ell h(s)\right\rangle\right) .
$$

Let us show that the system

$$
\left(\left\langle M_{2 \ell h(s)} \mid s \in{ }^{<\omega} \omega\right\rangle,\left\langle i_{2 \ell h\left(s_{1}\right), 2 \ell h\left(s_{2}\right)} \mid s_{1} \subseteq s_{2} \& s_{1}, s_{2} \in{ }^{<\omega} \omega\right\rangle\right)
$$

gives embedding normal form for $\neg p[T]$.

We must check property (c) in the definition of embedding normal form. Let $x \in{ }^{\omega} \omega$. Let $M_{k}=M_{k}(x \backslash n), E_{k}=E_{k}(x \backslash n)$, and $\rho_{k}=\rho_{k}(x \backslash n)$, where $n$ is large enough that these are defined. Let $\mathscr{C}$ be the alternating chain $\left(\left\langle M_{k} \mid k \in \omega\right\rangle,\left\langle E_{k} \mid k \in \omega\right\rangle,\left\langle\rho_{k} \mid k \in \omega\right\rangle\right)$. Recall that Even $=\{2 n \mid n \in \omega\}$. We must show that

$$
\begin{aligned}
& x \in p[T] \Rightarrow M_{\text {Even }} \text { is illfounded; } \\
& x \notin p[T] \Rightarrow M_{\text {Even }} \text { is wellfounded } .
\end{aligned}
$$

Assume first that $x \in p[T]$. By Lemma 1.1, there is an $f: \omega \rightarrow \lambda$ such that $(\forall k)\left(f \mid k \in X_{x \mid k}\right)$. For each $k \in \omega$, let $\beta_{k}=\beta_{k}(x|k, f| k)$. R5 implies that

$$
\beta_{k+1}<i_{2 k, 2 k+2}\left(\beta_{k}\right) \text {, }
$$

where the $i_{m, n}$ are the canonical elementary embeddings associated with $\mathscr{C}$. Thus $\left\langle i_{2 k}\right.$.Even $\left.\left(\beta_{k}\right) \mid k \in \omega\right\rangle$ is an infinite descending sequence of ordinals of $M_{\text {Even }}$.

Assume now that $x \notin p[T]$. Let $u_{k}=u_{k}(x \mid k)$. By R7, $u_{k+1} \in$ $i_{0,2 k+1}(T(x))$. Since $x \notin p[T], T(x)$ is wellfounded. Let

$$
\gamma_{k}=\operatorname{rank}_{i_{0.2 k+1}(T(x))}\left(u_{k+1}\right) \text {. }
$$

Since $i_{2 k+1,2 k+3}\left(u_{k+1}\right) \subsetneq u_{k+2}$, we have that $i_{2 k+1,2 k+3}\left(\gamma_{k}\right)>\gamma_{k+1}$. For each $k$ let $\xi_{2 k+1}=\gamma_{k}$. The $\xi_{n}, n \in \omega$-Even, satisfy the hypotheses of Lemma 3.2. Hence $M_{\text {Even }}$ is wellfounded.

Theorem 5.6. If $T$ is a $\delta^{+}$-homogeneous tree on $\omega \times \lambda, T^{*}$ is defined from $T$ as in $\S 2$, and $\delta$ is Woodin, then $(\forall \alpha<\delta)\left(T^{*}\right.$ is $\alpha$-homogeneous $)$.

Proof. Let $\left\langle\mu_{s} \mid s \in{ }^{<\omega} \omega\right\rangle$ witness that $T$ is $\delta^{+}$-homogeneous. Let $\alpha<\kappa_{0}<\delta$ so that $I I$ has a winning strategy $\tau$ for $\mathscr{G}_{\kappa_{0}}^{T}$. Let $M_{k}(s), E_{k}(s), \rho_{\kappa}(s)$, and $\beta_{\kappa}(s, t)$ be defined as in the proof of Theorem 5.5. Let $i_{m, n}(s)$ be the canonical elementary embedding associated with

$$
\left(\left\langle M_{k}(s) \mid k \leq 2 \ell h(s)\right\rangle,\left\langle E_{k}(s) \mid k<2 \ell h(s)\right\rangle,\left\langle\rho_{k}(s) \mid k<2 \ell h(s)\right\rangle\right),
$$

for $m \leq n \leq 2 \ell h(s)$ and either $m=0$ or else $n-m$ is even.

For $s \in{ }^{<\omega} \omega$ and $k<\ell h(s)$ define $f_{k}^{s}: T_{s \mid k} \rightarrow \mathrm{ON}$ by

$$
f_{k}^{s}(t)=\beta_{k}(s \mid k, t) \text {. }
$$


Let $e_{k}(s)=\llbracket f_{k}^{s} \rrbracket_{\mu_{s / k}}$. Define measures $\nu_{s}$ for $s \in{ }^{<\omega} \omega$ by

$$
\nu_{s}(X)=1 \Leftrightarrow\left\langle\left(i_{2 k, 2 \ell h(s)}(s)\right)\left(e_{k}(s)\right) \mid k<\ell h(s)\right\rangle \in\left(i_{0,2 \ell h(s)}(s)\right)(X) .
$$

R4 implies that $\kappa_{2 k+2}>\kappa_{2 k+1}>\alpha_{k}=\kappa_{2 k}$. Hence the critical points of the $E_{k}(s)$ are $\geq \kappa_{0}$, and the $\nu_{s}$ are all $\kappa_{0}$-complete and so are $\alpha$-complete. If $s_{1} \subseteq s_{2}$, then

$$
\begin{aligned}
\nu_{s_{1}}(X)=1 & \Leftrightarrow\left\langle\left(i_{2 k, 2 \ln \left(s_{1}\right)}\left(s_{1}\right)\right)\left(e_{k}\left(s_{1}\right)\right) \mid k<\operatorname{lh}\left(s_{1}\right)\right\rangle \in\left(i_{0,2 \ell h\left(s_{1}\right)}\left(s_{1}\right)\right)(X) \\
& \Leftrightarrow\left\langle\left(i_{2 k, 2 \ell h\left(s_{1}\right)}\left(s_{2}\right)\right)\left(e_{k}\left(s_{2}\right)\right) \mid k<\operatorname{lh}\left(s_{1}\right)\right\rangle \in\left(i_{0,2 \ell h\left(s_{1}\right)}\left(s_{2}\right)\right)(X) \\
& \Leftrightarrow\left\langle\left(i_{2 k, 2 \ell h\left(s_{2}\right)}\left(s_{2}\right)\right)\left(e_{k}\left(s_{2}\right)\right) \mid k<\operatorname{lh}\left(s_{1}\right)\right\rangle \in\left(i_{0,2 \ell h\left(s_{2}\right)}\left(s_{2}\right)\right)(X) \\
& \Leftrightarrow \nu_{s_{2}}\left(\left\{z|z| \ln \left(s_{1}\right) \in X\right\}\right)=1 .
\end{aligned}
$$

Furthermore, if $x \notin p[T]$, then the proof of Theorem 5.5 shows that $M_{\text {Even }}(x)$, the direct limit of $\left(\left\langle M_{2 k}(x \uparrow k) \mid k \in \omega\right\rangle,\left\langle i_{2 m, 2 n}(x \mid n+1) \mid m \leq n \in \omega\right\rangle\right)$, is wellfounded. By Lemma 1.2.(b), it follows that

$$
x \in p\left[T^{*}\right] \Rightarrow x \notin p[T] \Rightarrow \operatorname{Ult}\left(V ;\left\langle\nu_{x \mid n} \mid n \in \omega\right\rangle\right) \text { is wellfounded. }
$$

The theorem will be proved if we show that, for all $s \in{ }^{<\omega} \omega, \nu_{s}\left(T_{s}^{*}\right)=1$. Fix then $s \in{ }^{<\omega} \omega$. We must show that

$$
\nu_{s}\left(\left\{z \mid(\forall k)\left(k+1<\ell h(s) \Rightarrow z(k+1)<j_{k, k+1}(z(k))\right)\right\}\right)=1,
$$

where $j_{m, n}, m \leq n$, are the canonical elementary embeddings of $\operatorname{Ult}\left(V ; \mu_{s \mid m}\right)$ into $\operatorname{Ult}\left(V ; \mu_{s \mid n}\right)$. By the definition of $\nu_{s}$, this is equivalent to

$$
(\forall k)\left(k+1<\ell h(s) \Rightarrow\left(i_{2 k+2,2 \ell h(s)}\left(e_{k+1}\right)\right)<\left(i_{0,2 \ell h(s)}\left(j_{k, k+1}\right)\right)\left(i_{2 k, 2 \ell h(s)}\left(e_{k}\right)\right)\right) \text {, }
$$

where we abbreviate $e_{n}(s)$ by $e_{n}$ and $i_{m, n}(s)$ by $i_{m, n}$. Fix then $k$ with $k+1<\ell h(s)$. We must show that

$$
i_{2 k+2,2 \ell h(s)}\left(e_{k+1}\right)<\left(i_{0,2 \ell h(s)}\left(j_{k, k+1}\right)\right)\left(i_{2 k, 2 \ell h(s)}\left(e_{k}\right)\right) .
$$

By the elementarity of $i_{2 k+2,2 \ell h(s)}$, this is equivalent to

$$
e_{k+1}<\left(i_{0,2 k+2}\left(j_{k, k+1}\right)\right)\left(i_{2 k, 2 k+2}\left(e_{k}\right)\right) \text {. }
$$

Since $\mu_{s \nmid k}$ and $\mu_{s\lceil k+1}$ are $\delta^{+}$-complete, Lemma 3.3 gives that $\left(i_{0,2 k+2}\left(j_{k, k+1}\right)\right)$ $1 \mathrm{ON}=j_{k, k+1} \mid \mathrm{ON}$. What we must show is thus finally reduced to

$$
e_{k+1}<j_{k, k+1}\left(i_{2 k, 2 k+2}\left(e_{k}\right)\right) \text {. }
$$

On the other hand, R5 implies that $\beta_{k+1}(s, t)<i_{2 k, 2 k+2}\left(\beta_{k}(s, t)\right)$ for all $t \in T_{s}$, and so $\beta_{k+1}(s \uparrow k+1, t)<i_{2 k, 2 k+2}\left(\beta_{k}(s \uparrow k, t \uparrow k)\right)$ for all $t \in T_{s \uparrow k+1}$. By the definition of $f_{k+1}^{s}$ and $f_{k}^{s}$,

$$
f_{k+1}^{s}(t)<i_{2 k, 2 k+2}\left(f_{k}^{s}(t \uparrow k)\right)
$$

for all $t \in T_{s \mid k+1}$. Thus $\llbracket f_{k+1}^{s} \rrbracket_{\mu_{s \mid k+1}}<j_{k, k+1}\left(\llbracket i_{2 k, 2 k+2} \circ f_{k}^{s} \rrbracket_{\mu_{s \mid k}}\right)$. Since $\llbracket i_{2 k, 2 k+2} \circ f_{k}^{s} \rrbracket_{\mu_{s\} k}}=\left(j_{0, k}\left(i_{2 k, 2 k+2}\right)\right)\left(\llbracket f_{k}^{s} \rrbracket_{\mu_{s\} k}}\right)$, we get

$$
\llbracket f_{k+1}^{s} \rrbracket_{\mu_{s \mid k+1}}<j_{k, k+1}\left(\left(j_{0, k}\left(i_{2 k, 2 k+2}\right)\right)\left(\llbracket f_{k}^{s} \rrbracket_{\mu_{s \mid k}}\right)\right) \text {. }
$$


By the definitions of $e_{k}$ and $e_{k+1}$, this means that

$$
e_{k+1}<j_{k, k+1}\left(\left(j_{0, k}\left(i_{2 k, 2 k+2}\right)\right)\left(e_{k}\right)\right) \text {. }
$$

Applying Lemma 3.3 again, we get

$$
e_{k+1}<j_{k, k+1}\left(i_{2 k, 2 k+2}\left(e_{k}\right)\right) \text {. }
$$

Since this is exactly what we are trying to show, the proof is complete.

Our remaining task is to extend the results obtained thus far in this section for the operation $T \mapsto T^{*}$ to corresponding results for the operation $T \mapsto \widetilde{T}$. For this we need to build iteration trees which have, instead of a single infinite branch in addition to Even, branches $b_{y}$ for each $y \in{ }^{\omega} \omega$. To do this we have to amalgamate a tree of alternating chain constructions of the sort already done. For this we require a modification of the game $\mathscr{G}_{\kappa_{0}}^{T}$. For this in turn we need a slight generalization of the notion of an alternating chain.

A pseudoalternating chain on $V$ of length $2 n+1$ is a system $\left(\left\langle M_{k}\right| k \leq\right.$ $\left.2 n\rangle,\left\langle E_{k} \mid k<2 n\right\rangle,\left\langle\rho_{k} \mid k<2 n\right\rangle,\left\langle\widehat{M}_{2 k} \mid k<n\right\rangle,\left\langle\hat{i}_{2 k} \mid k<n\right\rangle\right)$ such that

(1) for all $k \leq 2 n, M_{k}$ is a transitive proper class model of ZFC; $M_{0}=V$; for all $\kappa<n, \widehat{M}_{2 k}$ is a transitive proper class model of $\mathrm{ZFC}$;

(2) $\left\langle\rho_{k} \mid k<2 n\right\rangle$ is a nondecreasing sequence of ordinals;

(3) for all $k<n, V_{\rho_{2 k}+1} \cap \widehat{M}_{2 k}=V_{\rho_{2 k+1}} \cap M_{2 k+1}$; for all $k<n V_{\rho_{2 k+1}+1} \cap$ $M_{2 k+1}=V_{\rho_{2 k+1}+1} \cap M_{2 k+2}$; and, for all $k$ with $k+1<n, V_{\rho_{2 k+1}+1} \cap$ $M_{2 k+2}=V_{\rho_{2 k+1}+1} \cap \widehat{M}_{2 k+2}$;

(4) for all $k<n, \widehat{M}_{2 k}$ satisfies " $E_{2 k}$ is an extender"; and, for all $k<n$, $M_{2 k+1}$ satisfies " $E_{2 k+1}$ is an extender";

(5) for all $k<n, \operatorname{support}\left(E_{2 k}\right) \supseteq V_{p_{2 k}+1} \cap \widehat{M}_{2 k}$; and, for all $k<n$, $\operatorname{support}\left(E_{2 k+1}\right) \supseteq V_{\rho_{2 k+1}+1} \cap M_{2 k+1}$;

(6) for all $k<n, \operatorname{crit}\left(E_{2 k}\right) \leq \rho_{2 k-1}$ and $M_{2 k+1}=\operatorname{Ult}\left(M_{2 k-1} ; E_{2 k}\right)$; and, for all $k<n, \operatorname{crit}\left(E_{2 k+1}\right) \leq \rho_{2 k}$ and $M_{2 k+2}=\operatorname{Uit}\left(\widehat{M}_{2 k} ; E_{2 k+1}\right)$;

(7) for all $k<n, \hat{i}_{2 k}: M_{2 k} \rightarrow \widehat{M}_{2 k}$ is an elementary embedding (which may be the identity, in which case $\widehat{M}_{2 k}=M_{2 k}$ );

(8) for all $k$ with $0<k<n, \operatorname{crit}\left(\hat{i}_{2 k}\right)>\rho_{2 k-1}$. (If $\hat{i}_{2 k}$ is the identity then we declare $\operatorname{crit}\left(\hat{i}_{2 k}\right)=\infty$, with $\infty>$ any ordinal number.)

Remark. A pseudoalternating chain on $V$ of length $2 n+1$ is just an alternating chain on $V$ of length $2 n+1$ with the extra "links" $M_{2 k} \stackrel{\hat{i}_{2 k}}{\longrightarrow} \widehat{M}_{2 k}$ interposed:

$$
\begin{aligned}
& V=M_{0} \stackrel{\hat{i}_{0}}{\longrightarrow} \widehat{M}_{0} \stackrel{i_{l_{1}}^{\widehat{u}_{0}}}{\longrightarrow} M_{2} \stackrel{\hat{i}_{2}}{\longrightarrow} \widehat{M}_{2} \rightarrow \quad \cdots \stackrel{\hat{i}_{l_{2 n-1}}^{\hat{n}_{2 n-2}}}{\longrightarrow} M_{2 n} \\
& i_{E_{0}}^{M_{0} \searrow} \\
& M_{1} \stackrel{i_{E_{2}}^{M_{1}}}{\longrightarrow} \quad M_{3} \rightarrow \quad \cdots \stackrel{i_{E_{2 n-2}}^{i_{2 n-3}}}{\longrightarrow} M_{2 n-1}
\end{aligned}
$$


when $n>0$. If all the $\hat{i}_{2 k}$ are identities, then we have, in essence, an ordinary alternating chain. Note that condition (8) guarantees that the last clause of (3) holds.

Let us say that a pseudoalternating chain on $V$ of length $2 n+1$ is countably closed if each $\widehat{M}_{2 k}$ satisfies "support $\left(E_{2 k}\right)$ is countably closed," each $M_{2 k+1}$ satisfies "support $\left(E_{2 k+1}\right)$ is countably closed", and each $\widehat{M}_{2 k}$ is countably closed.

We have the following analogue of Lemma 3.1.

Lemma 5.7. Let $\left(\left\langle M_{k} \mid k \leq 2 n\right\rangle,\left\langle E_{k} \mid k<2 n\right\rangle,\left\langle\rho_{k} \mid k<2 n\right\rangle,\left\langle\widehat{M}_{2 k} \mid k<n\right\rangle\right.$, $\left.\left\langle\hat{i}_{2 k} \mid k<n\right\rangle\right)$ be a countably closed pseudoalternating chain on $V$. Let $\widehat{M}$ be a countably closed proper class model of ZFC and let $\hat{i}: M_{2 n} \rightarrow \widehat{M}$ be an elementary embedding such that $\operatorname{crit}(\hat{i})>\rho_{2 n-1}$ if $n>0$. Let $\rho$ be an ordinal with $\rho \geq \rho_{2 n-1}$ if $n>0$. Let $E \in \widehat{M}$ be such that $\widehat{M}$ satisfies " $E$ is an extender with countably closed support " and such that $\operatorname{support}(E) \supseteq V_{\rho+1} \cap \widehat{M}$. Assume that $\operatorname{crit}(E) \leq \rho_{2 n-1}$ if $n>0$ and that $\operatorname{crit}(E) \leq \max (\rho, \operatorname{crit}(\hat{i}))$ if $n=0$. Let $\rho^{*}$ be an ordinal $\geq \rho$. Let $E^{*} \in \operatorname{Ult}\left(M_{2 n-1} ; E\right)$ be such that $\operatorname{Ult}\left(M_{2 n-1} ; E\right)$ satisfies " $E^{*}$ is an extender with countably closed support" and such that $\operatorname{support}\left(E^{*}\right) \supseteq V_{\rho^{*}+1} \cap \operatorname{Ult}\left(M_{2 n-1} ; E\right)$. Assume that $\operatorname{crit}\left(E^{*}\right) \leq \rho$.

There is a unique countably closed pseudoalternating chain $\left(\left\langle M_{k}^{\prime}\right| k \leq 2 n+\right.$ 2), $\left.\left\langle E_{k}^{\prime} \mid k<2 n+2\right\rangle,\left\langle\rho_{k}^{\prime} \mid k<2 n+2\right\rangle,\left\langle\widehat{M}_{2 k}^{\prime} \mid k \leq n\right\rangle,\left\langle\hat{i}_{2 k}^{\prime} \mid k \leq n\right\rangle\right)$ on $V$ of length $2 n+3$ with $M_{k}^{\prime}=M_{k}$ for all $k \leq 2 n, E_{k}^{\prime}=E_{k}$ and $\rho_{k}^{\prime}=\rho_{k}$ for all $k<2 n, \widehat{M}_{2 k}^{\prime}=\widehat{M}_{2 k}$ and $\hat{i}_{2 k}^{\prime}=\hat{i}_{2 k}$ for all $k<n, \widehat{M}_{2 n}^{\prime}=\widehat{M}, \quad \hat{i}_{2 n}^{\prime}=\hat{i}$, $E_{2 n}^{\prime}=E, \rho_{2 n}^{\prime}=\rho, E_{2 n+1}^{\prime}=E^{*}$, and $\rho_{2 n+1}^{\prime}=\rho^{*}$.

The proof of Lemma 5.7 is like that of Lemma 3.1. The only new point is that the fact that $\operatorname{crit}(\hat{i})>\rho_{2 n-1}$, which guarantees (8), implies also that $V_{\rho_{2 n-1}+1} \cap M_{2 n}=V_{\rho_{2 n-1}+1} \cap \widehat{M}$ as required by the last clause of (3).

For trees $T$ on $(\omega \times \omega) \times \lambda$ and ordinals $\kappa_{0}<\delta$, we consider the following game $\tilde{\mathscr{G}}_{\kappa_{0}}^{T}$ :

$$
\begin{aligned}
& I\left\langle m_{0}, p_{0}, \eta_{0}, \alpha_{0}, \widehat{M}_{0}, \hat{i}_{0}\right\rangle \quad\left\langle m_{1}, p_{1}, \eta_{1}, \alpha_{1}, \widehat{M}_{2}, \hat{i}_{2}\right\rangle \quad \ldots \\
& I I\left\langle E_{0}, \rho_{0}, \eta_{0}^{\prime}, \kappa_{1}, E_{1}, \rho_{1}, \beta_{1}, \kappa_{2}\right\rangle \quad\left\langle E_{2}, \rho_{2}, \eta_{1}^{\prime}, \kappa_{3}, E_{3}, \rho_{3}, \beta_{2}, \kappa_{4}\right\rangle \quad \ldots
\end{aligned}
$$

The first player to disobey one of the following rules loses a play of $\tilde{\mathscr{G}}_{\kappa_{0}}^{T}$. If all rules are obeyed, $I I$ wins.

$\widetilde{\mathrm{R}} 1$. The $E_{n}, \rho_{n}, \hat{i}_{2 n}$, and $\widehat{M}_{2 n}$ generate a countably closed pseudoalternating chain on $V$ with all $E_{n} \in V_{\delta}$ and with $\operatorname{crit}\left(\hat{i}_{0}\right)>\kappa_{0}, \hat{i}_{2 n}(\delta)=\delta$, and $(\forall k \leq 2)\left(\hat{i}_{2 n}\left(c_{k}\right)=c_{k}\right)$.

As long as $\widetilde{\mathrm{R}} 1$ is obeyed define $M_{n}$ and $i_{m, n}: M_{m} \rightarrow M_{n}$ in the obvious way. Note that $\widetilde{R} 1$-unlike $\mathrm{R} 1$ of $\mathscr{G}_{\kappa_{0}}^{T}$-is a constraint on the moves of $I$ as well as 
of $I I$. In particular, $I$ violates $\widetilde{\mathrm{R}} 1$ if he does not choose $\hat{i}_{2 n}: M_{2 n} \rightarrow \widehat{M}_{2 n}$ as an elementary embedding or fails to have $\operatorname{crit}\left(\hat{i}_{2 n+2}\right)>\rho_{2 n+1}$.

$\widetilde{R} 2, \widetilde{R} 3, \widetilde{R} 4, \widetilde{R} 5$, and $\widetilde{R} 8$ are word-for-word repetitions of $R 2, R 3, R 4$, $\mathrm{R} 5$, and $\mathrm{R} 8$ respectively. $\widetilde{\mathrm{R}} 6$ and $\widetilde{\mathrm{R}} 7$ are the natural variants of $\mathrm{R} 6$ and $\mathrm{R} 7$ respectively:

$$
\begin{aligned}
\widetilde{\mathrm{R}} 6 \text {. Let } t_{n}= & \left\langle i_{2 k, 2 n-2}\left(\eta_{k}\right) \mid k<n\right\rangle . \\
& \left\langle\left\langle\left\langle m_{k} \mid k \leq n\right\rangle,\left\langle p_{k} \mid k \leq n\right\rangle\right\rangle, t_{n+1}\right\rangle \in i_{0,2 n}(T) .
\end{aligned}
$$

$\widetilde{\mathrm{R}} 7$. Let $u_{n}=\left\langle i_{2 k+1,2 n-1}\left(\eta_{k}^{\prime}\right) \mid k<n\right\rangle$.

$$
\left\langle\left\langle\left\langle m_{k} \mid k \leq n\right\rangle,\left\langle p_{k} \mid k \leq n\right\rangle\right\rangle, u_{n+1}\right\rangle \in i_{0,2 n+1}(T) .
$$

Here, or rather in the following Lemma 5.8, we are passing beyond the bounds of Kelley-Morse set theory, since a strategy for $I I$ has to be of a higher type than a proper class. In the application of Lemma 5.8, each $\hat{i}_{2 n}: M_{2 n} \rightarrow \widehat{M}_{2 n}$ will be $\tilde{i}_{p, q}: \widetilde{M}_{p} \rightarrow \widetilde{M}_{q}$ for some iteration tree $\mathscr{T} \in V_{\delta}$ with models $\widetilde{M}_{k}$ and canonical embeddings $\tilde{i}_{p, q}$. The careful reader will then want to think of $\widetilde{\mathrm{R}} 1$ as strengthened to demand that $\hat{i}_{2 n}$ and $\widehat{M}_{2 n}$ are of this sort, so that $I$ 's moves may be thought of as sets.

Lemma 5.8. If $\delta$ is Woodin and $T$ is a tree on $(\omega \times \omega) \times \lambda$, then the set of $\kappa_{0}<\delta$ such that II has a winning strategy for $\tilde{\mathscr{G}}_{\kappa_{0}}^{T}$ is unbounded in $\delta$.

Proof. As in the proof of Lemma 5.2, we let $\kappa_{0}<\delta$ be $\left(c_{0}+1\right)$-reflecting in $\langle T\rangle$, and we construct a winning strategy $\tau$ for $I I$ for $\tilde{\mathscr{G}}_{\kappa_{0}}^{T}$.

The construction of $\tau$ is almost the same as the construction in the proof of Lemma 5.4. Assume inductively that we have defined $\tau$ on all positions of lengths $<2 n$ and that we are given a position of length $2 n$ which is consistent with $\tau$ and is such that neither player has disobeyed the rules. Assume inductively $(*)_{n},(* *)_{n}$, and $(* * *)_{n}$-word for word repetitions of the corresponding assertions in the proof of Lemma 5.4. As before, $\beta_{0}=c_{0}$.

Let $I$ play $\left\langle m_{n}, p_{n}, \alpha_{n}, \widehat{M}_{2 n}, \hat{i}_{2 n}\right\rangle$ and assume that this move obeys all relevant rules. Thus $\alpha_{n}<\delta$ and $t_{n+1}=i_{2 n-2,2 n}\left(t_{n}\right)^{-}\left\langle\eta_{n}\right\rangle$ is such that

$$
\left\langle\left\langle\left\langle m_{k} \mid k \leq n\right\rangle,\left\langle p_{k} \mid k \leq n\right\rangle\right\rangle, t_{n+1}\right\rangle \in i_{0,2 n}(T) .
$$

Furthermore $\widehat{M}_{2 n}$ is a countably closed proper class model of ZFC, $\hat{i}_{2 n}: M_{2 n} \rightarrow$ $\widehat{M}_{2 n}$ is elementary, $\operatorname{crit}\left(\hat{i}_{2 n}\right)>\rho_{2 n-1}$ if $n>0, \operatorname{crit}\left(\hat{i}_{2 n}\right)>\kappa_{0}$ if $n=0$, $\hat{i}_{2 n}(\delta)=\delta$, and, for all $k \leq 2, \hat{i}_{2 n}\left(c_{k}\right)=c_{k}$.

Thus $\left\langle\left\langle\left\langle m_{k} \mid k \leq n\right\rangle,\left\langle p_{k} \mid k \leq n\right\rangle\right\rangle, \hat{i}_{2 k}\left(t_{n+1}\right)\right\rangle \in \hat{i}_{2 n}\left(i_{0,2 n}(T)\right)$. Furthermore we have, since $\operatorname{crit}\left(\hat{i}_{2 n}\right)>\rho_{2 n-1}$ if $n>0$ and $\operatorname{crit}\left(i_{2 n}\right)>\kappa_{0}$ if $n=0$, that $\kappa_{2 n}<\operatorname{crit}\left(\hat{i}_{2 n}\right)$. This gives us the following.

$(* *)_{n}^{\wedge}$ The $\left(\kappa_{2 n}, \hat{i}_{2 n}\left(\beta_{n}\right)+1\right)$-type of $\left\langle\hat{i}_{2 n}\left(i_{0.2 n}(T)\right\rangle^{-} \hat{i}_{2 n}\left(i_{2 n-2,2 n}\left(t_{n}\right)\right)\right.$ in $\widehat{M}_{2 n}$ is the same as the $\left(\kappa_{2 n}, c_{0}+1\right)$-type of $\left\langle i_{0,2 n-1}(T)\right\rangle^{-} u_{n}$ in $M_{2 n-1}$. 
$(* * *)_{n}^{\wedge} \quad \begin{gathered}\kappa_{2 n} \\ \widehat{M}_{2 n}\end{gathered}$ is $\left(\hat{i}_{2 n}\left(\beta_{n}\right)+1\right)$-reflecting in $\left\langle\hat{i}_{2 n}\left(i_{0,2 n}(T)\right)\right\rangle-\hat{i}_{2 n}\left(i_{2 n-2,2 n}\left(t_{n}\right)\right)$ in

The assumptions of the One-Step Lemma hold with $M=\widehat{M}_{2 n}, N=M_{2 n-1}$, $\kappa=\kappa_{2 n}, \eta=\alpha_{n}, \quad \beta=\hat{i}_{2 n}\left(\beta_{n}\right)+1, \beta^{\prime}=c_{0}+1, \xi=\hat{i}_{2 n}\left(\beta_{n}\right), x=$ $\left\langle\hat{i}_{2 n}\left(i_{0,2 n}(T)\right)\right\rangle \hat{i}_{2 n}\left(i_{2 n-2,2 n}\left(t_{n}\right)\right), x^{\prime}=\left\langle i_{0,2 n-1}(T)\right\rangle u_{n}, \quad y=\left\langle\eta_{n}\right\rangle$, and $\varphi(v)$ as " $\delta+v$ is the largest ordinal." $(*)_{n}$ and the fact that $\widetilde{\mathrm{R}} 1$ has been obeyed give $(\mathrm{a}),(* *)_{n}^{\wedge}$ gives $(\mathrm{b})$, and $(* * *)_{n}^{\wedge}$ gives $(\mathrm{c}) .(\mathrm{d})$ is obvious.

Let $E, x^{*}, \xi^{*}$, and $y^{*}$ be given by the One-Step Lemma. $y^{*}$ is a sequence of length one and $\xi^{*}=c_{0}$.

Let $\tau$ call for $I I$ to play

$$
E_{2 n}=E, \quad \rho_{2 n}=\kappa^{*}, \quad\left\langle\eta_{n}^{\prime}\right\rangle=y^{*}, \text { and } \kappa_{2 n+1}=\kappa^{*} .
$$

As in the proof of Lemma 5.4, $\widetilde{R} 1$ is obeyed. As in that proof we get the following.

$(*)_{n}^{\prime} \quad \kappa_{2 n+1} \leq \rho_{2 n}$.

$(* *)^{\wedge \prime}$ The $\left(\kappa_{2 n+1}, c_{0}\right)$-type of $\left\langle i_{0,2 n+1}(T)\right\rangle^{\wedge} u_{n+1}$ in $M_{2 n+1}$ is the same as the $\left(\kappa_{2 n+1}, \hat{i}_{2 n}\left(\beta_{n}\right)\right)$-type of $\left\langle\hat{i}_{2 n}\left(i_{0,2 n}(T)\right)\right\rangle \hat{i}_{2 n}\left(t_{n+1}\right)$ in $\widehat{M}_{2 n}$.

$(* * *)_{n}^{\prime} \quad \kappa_{2 n+1}$ is $c_{0}$-reflecting in $\left\langle i_{0,2 n+1}(T)\right\rangle u_{n+1}$ in $M_{2 n+1}$.

As in the proof of Lemma $5.4, \widetilde{R} 2, \widetilde{R} 4, \widetilde{R} 7$, and $\widetilde{R} 8$ are obeyed.

As in the proof of Lemma 5.4, we get the following.

$(* *)_{n}^{\wedge \prime \prime}$ The $\left(\kappa_{2 n+1}, c_{1}\right)$-type of $\left\langle i_{0,2 n+1}(T)\right\rangle-u_{n+1}$ in $M_{2 n+1}$ is the same as the $\left(\kappa_{2 n+1}, \hat{i}_{2 n}\left(\beta_{n}\right)\right)$-type of $\left\langle\hat{i}_{2 n}\left(i_{0,2 n}(T)\right)\right\rangle^{-} \hat{i}_{2 n}\left(t_{n+1}\right)$ in $\widehat{M}_{2 n}$.

$(* * *)_{n}^{\wedge \prime \prime} \kappa_{2 n+1}$ is $c_{1}$-reflecting in $\left\langle i_{0}, 2 n+1(T)\right\rangle^{-} u_{n+1}$ in $M_{2 n+1}$.

The assumptions of the One-Step Lemma hold with $M=M_{2 n+1}, N=$ $\widehat{M}_{2 n}, \kappa=\kappa_{2 n+1}, \eta=\kappa_{2 n+1}, \quad \beta=c_{1}, \beta^{\prime}=\hat{i}_{2 n}\left(\beta_{n}\right), \xi=c_{0}+1, x=$ $\left\langle i_{0,2 n+1}(T)\right\rangle u_{n+1}, x^{\prime}=\left\langle\hat{i}_{2 n}\left(i_{0,2 n}(T)\right)\right\rangle-\hat{i}_{2 n}\left(t_{n+1}\right), y=\varnothing$, and $\varphi(v)$ the trivial formula $v=v$.

Let $E, \kappa^{*}, \xi^{*}$, and $y^{*}$ be given by the One-Step Lemma. $y^{*}=\varnothing . \xi^{*}$ is a successor ordinal.

Let $\tau$ call for $I I$ to play $E_{2 n+1}=E, \rho_{2 n+1}=\kappa^{*}, \beta_{n+1}+1=\xi^{*}$, and $\kappa_{2 n+2}=\kappa^{*}$. As in the proof of Lemma 5.4, $\widetilde{\mathrm{R}} 1, \widetilde{\mathrm{R}} 2$, and $\widetilde{\mathrm{R}} 5$ are obeyed and $(*)_{n+1},(* *)_{n+1}$, and $(* * *)_{n+1}$ hold.

Let $\prec$ be the following partial ordering.

(i) $0 \prec n$ for all $n>0$.

(ii) $2 m \prec 2 n \Leftrightarrow m<n$.

(iii) $2 m+1 \prec 2 n+1 \Leftrightarrow r_{m+1} \subsetneq r_{n+1}$, where $\left\langle r_{k} \mid k \in \omega\right\rangle$ is the enumeration of ${ }^{<\omega} \omega$ in $\S 2$.

(iv) $m \prec n$ only if (i), (ii), or (iii) require $m \prec n$.

Note that $(\omega, \prec)$ is a tree. 
Lemma 5.9. Let $T$ be a tree on $(\omega \times \omega) \times \lambda$, let $\kappa_{0}<\lambda$, let $\tau$ be a winning strategy for II for $\tilde{\mathscr{G}}_{\kappa_{0}}^{T}$, and let $\left\langle\mu_{\langle s, r\rangle} \mid s \in{ }^{<\omega} \omega \& r \in{ }^{<\omega} \omega \& \ln (s)=\ell h(r)\right\rangle$ witness that $T$ is $\delta^{+}$-homogeneous.

There are $\left\langle\mathscr{T}_{s} \mid s \in{ }^{<\omega} \omega\right\rangle,\left\langle X_{\langle s, r\rangle} \mid s \in{ }^{<\omega} \omega \& r \in{ }^{<\omega} \omega \& \ln (s)=\operatorname{lh}(r)\right\rangle$, and $\left\langle P_{\langle s, r, t\rangle} \mid s \in{ }^{<\omega} \omega \& r \epsilon^{<\omega} \omega \& \ell h(s)=\ell h(r) \& t \in X_{\langle s, r\rangle}\right\rangle$ such that

$$
\begin{aligned}
& \mathscr{T}_{s}=(<\mid 2 \ell h(s)+1,\left\langle\widetilde{M}_{k}(s) \mid k \leq 2 \ell h(s)\right\rangle, \\
&\left.\left\langle\widetilde{E}_{k}(s) \mid k<2 \ell h(s)\right\rangle,\left\langle\tilde{\rho}_{k}(s) \mid k<2 \ell h(s)\right\rangle\right)
\end{aligned}
$$

is a countably closed iteration tree on $V$ of length $2 \ell h(s)+1, \mathscr{T}_{s_{1}}$, extends $\mathscr{T}_{s_{2}}$ if $s_{1}$ extends $s_{2}, \mu_{\langle s, r\rangle}\left(X_{\langle s, r\rangle}\right)=1, P_{\langle s, r, t\rangle}$ is a position of length $2 \theta(r) \stackrel{s_{2}}{=}$ $2 \max \left\{\ell h\left(r_{q}\right) \mid r_{q} \subseteq r \& q \leq \ell h(r)\right\}$ in $\tilde{\mathscr{G}}_{\kappa_{0}}^{T}$ with all rules obeyed, and the following conditions are met.

(a) If $\langle s, r, t\rangle$ extends $\left\langle s^{\prime}, r^{\prime}, t^{\prime}\right\rangle$, then $P_{\langle s, r, t\rangle}$ extends $P_{\left\langle s^{\prime}, r^{\prime}, t^{\prime}\right\rangle}$. Moreover if $\theta(r) \geq k$ and $r\left|k=r^{\prime}\right| k$ and $t\left|k=t^{\prime}\right| k$ (and $t \in X_{\langle s, r\rangle}$ and $\left.t^{\prime} \in X_{\left\langle s, r^{\prime}\right\rangle}\right)$ then the first $2 k$ moves of $P_{\langle s, r, t\rangle}$ are the same as those of $P_{\left\langle s, r^{\prime}, t^{\prime}\right\rangle}$.

(b) $m_{k}(s, r, t)=s(k), p_{k}(s, r, t)=r(k), \eta_{k}(s, r, t)=\left(i_{0,2 k}(s, r, t)\right)(t(k))$. (Where $m_{k}(s, r, t)$, etc., are the appropriate moves in $P_{\langle s, r, t\rangle}$ )

(c) If $r_{1} \subseteq r$, then $\alpha_{0}(s, r, t)=\kappa_{0}$. If $r_{k+1} \subseteq r$ and $0<k<\ell h(r)$, then $\alpha_{\ell h\left(r_{k+1}\right)-1}(s, r, t)=\tilde{\rho}_{2 k-1}(s)$.

(d) If $r_{k+1} \subseteq r$ and $k<\operatorname{lh}(r)$, then $\widehat{M}_{2 \ell h\left(r_{k+1}\right)-2}(s, r, t)=\widetilde{M}_{2 k}(s)$, $\hat{i}_{2 \ell h\left(r_{k+1}\right)-2}(s, r, t)=\tilde{i}_{0,2 k}(s)$ if $\ln \left(r_{k+1}\right)=1$, and $\hat{i}_{2 \ell h\left(r_{k+1}\right)-2}(s, r, t)=$ $\tilde{i}_{2 k^{\prime}+2,2 k}(s)$ if $k^{\prime}<k$ is maximal such that $r_{k^{\prime}+1} \subseteq r_{k+1}$. (Here $\tilde{i}_{m, n}(s)$, $m \preceq n \leq 2 \ell h(s)$, are the canonical elementary embeddings associated with $\left.\mathscr{T}_{s}.\right)$

(e) $P_{\langle s, r, t\rangle}$ is consistent with $\tau$.

(f) If $r_{k+1} \subseteq r$ and $k<\ell h(r)$, then

$$
\begin{aligned}
E_{2 \ell h\left(r_{k+1}\right)-2}(s, r, t) & =\widetilde{E}_{2 k}(s) ; \\
\rho_{2 \ell h\left(r_{k+1}\right)-2}(s, r, t) & =\tilde{\rho}_{2 k}(s) ; \\
M_{2 \ell h\left(r_{k+1}\right)-1}(s, r, t) & =\widetilde{M}_{2 k+1}(s) ; \\
E_{2 \ell h\left(r_{k+1}\right)-1}(s, r, t) & =\widetilde{E}_{2 k+1}(s) ; \\
\rho_{2 \ell h\left(r_{k+1}\right)-1}(s, r, t) & =\tilde{\rho}_{2 k+1}(s) ; \\
M_{2 \ell h\left(r_{k+1}\right)}(s, r, t) & =\widetilde{M}_{2 k+2}(s) .
\end{aligned}
$$

Proof. Fix $k \in \omega$. Assume that we have defined all our objects for all $s$ and $r$ whose lengths are $\leq k$ and that all our conditions are satisfied. (In the case $k=0$ this can be done trivially.) 
Now fix $s$ with $\ell h(s)=k+1$. Let $\widetilde{M}_{n}(s)=\widetilde{M}_{n}(s \mid k)$ for $n \leq 2 k$; let $\widetilde{E}_{n}(s)=\widetilde{E}_{n}(s \uparrow k)$ and $\tilde{\rho}_{n}(s)=\tilde{\rho}_{n}(s \uparrow k)$ for $n<2 k$ (i.e., let $\mathscr{T}_{s}$ extend $\mathscr{T}_{s \uparrow k}$, as required).

Let us pause to calculate lower bounds for $\operatorname{crit}\left(\tilde{i}_{2 n, 2 n+2}(s)\right)$ for $n<k$. By definition this is $\operatorname{crit}\left(\widetilde{E}_{2 n+1}(s)\right)$. By (f) and the fact that $\widetilde{E}_{2 n+1}(s)=$ $\widetilde{E}_{2 n+1}(s \uparrow k), \widetilde{E}_{2 n+1}(s)=E_{2 \ell h\left(r_{n+1}\right)-1}(s \uparrow k, r, t)$ for $r_{n+1} \subseteq r$ and $t \in$ $X_{\langle s \mid k, r\rangle}$. Fix such $r$ and $t . \operatorname{crit}\left(\tilde{i}_{2 n, 2 n+2}(s)\right)=\operatorname{crit}\left(E_{2 \ell h\left(r_{n+1}\right)-1}(s \mid k, r, t)\right)=$ $\kappa_{2 \ell h\left(r_{n+1}\right)-1}(s \uparrow k, r, t)$. $\widetilde{\mathrm{R}} 4$ gives that

$$
\kappa_{2 \ell h\left(r_{n+1}\right)-1}(s \mid k, r, t)>\alpha_{\ell h\left(r_{n+1}\right)-1}(s \mid k, r, t) .
$$

Hence $\operatorname{crit}\left(\tilde{i}_{2 n, 2 n+2}(s)\right)>\alpha_{\ell h\left(r_{n+1}\right)-1}(s \mid k, r, t)$. Thus the case $n=0$ gives

$$
\operatorname{crit}\left(\tilde{i}_{0,2}(s)\right)>\alpha_{0}(s \uparrow k, r, t)=\kappa_{0},
$$

and, for $n>0$

$$
\operatorname{crit}\left(\tilde{i}_{2 n, 2 n+2}(s)\right)>\tilde{\rho}_{2 n-1}(s)
$$

(In both cases we have used (c).)

Now fix $r$ with $\ell h(r)=\ell h(s)=k+1$.

If $r_{k+1} \nsubseteq r$ then $\theta(r)=\theta(r \mid k)$, and so we let $X_{\langle s, r\rangle}=X_{\langle s|k, r| k\rangle}$ and let $P_{\langle s, r, t\rangle}=P_{\langle s|k, r| k, t \mid k\rangle}$ for $t \in X_{\langle s, r\rangle}$.

Assume then that $r_{k+1} \subseteq r . X_{\langle s, r\rangle}$ will project to a subset of $X_{\langle s|k, r| k\rangle}$ (i.e., $X_{\langle s, r\rangle} \subseteq\left\{t|t| k \in X_{\langle|k, r| k\rangle}\right\}$. We shall actually define $P_{\langle s, r, t\rangle}$ for all $t$ such that $t \mid k \in X_{\langle s|k, r| k\rangle}$.

For $t \in X_{\langle s|k, r| k\rangle}$, let $m_{\ell h\left(r_{k+1}\right)-1}(s, r, t), p_{\ell h\left(r_{k+1}\right)-1}(s, r, t), \eta_{\ell h\left(r_{k+1}\right)-1}(s, r, t)$, $\alpha_{\ell h\left(r_{k+1}\right)-1}(s, r, t), \widehat{M}_{2 \ell h\left(r_{k+1}\right)-2}(s, r, t)$, and $\hat{i}_{2 \ell h\left(r_{k+1}\right)-2}(s, r, t)$ be given by (b), (c), and (d).

Since $t \in T_{\langle s, r\rangle}$, this move obeys $\widetilde{R} 6$. It is easy to see that it obeys all the requirements of $\widetilde{\mathrm{R}} 1$ except perhaps those that $\operatorname{crit}\left(\hat{i}_{2 n+2}\right)>\rho_{2 n+1}$ and that $\operatorname{crit}\left(\hat{i}_{0}\right)>\kappa_{0}$.

Assume first that $\ell h\left(r_{k+1}\right)=1$. We must show that $\operatorname{crit}\left(\hat{i}_{0}(s, r, t)\right)>\kappa_{0}$. If $k>0$ then $\operatorname{crit}\left(\hat{i}_{0}(s, r, t)\right)=\operatorname{crit}\left(\tilde{i}_{0,2 k}(s)\right)=\inf \left\{\operatorname{crit}\left(\tilde{i}_{2 n, 2 n+2}(s)\right) \mid n<k\right\}>$ $\inf \left\{\tilde{\rho}_{2 n-1}(s) \mid 0<n<k\right\} \geq \tilde{\rho}_{0}(s) \geq \kappa_{0}$. If $k=0$ then $\hat{i}_{0}$ is the identity map, so $\operatorname{crit}\left(\hat{i}_{0}\right)=\infty>\kappa_{0}$.

Now assume that $\ell h\left(r_{k+1}\right)>1$. We must show that $\operatorname{crit}\left(\hat{i}_{2 \ell h\left(r_{k+1}\right)-2}(s, r, t)\right)$ $>\rho_{2 \ell h\left(r_{k+1}\right)-3}(s, r, t)$. Now $\hat{i}_{2 \ell h\left(r_{k+1}\right)-2}(s, r, t)=\tilde{i}_{2 k^{\prime}+2,2 k}(s)$, where $k^{\prime}<k$ is maximal such that $r_{k^{\prime}+1} \subset r_{k+1}$. So

$$
\begin{aligned}
\operatorname{crit}\left(\hat{i}_{2 \ell h\left(r_{k+1}\right)-2}(s, r, t)\right) & =\inf \left\{\operatorname{crit}\left(\tilde{i}_{2 n, 2 n+2}(s)\right) \mid k^{\prime}<n<k\right\} \\
& >\inf \left\{\tilde{\rho}_{2 n-1}(s) \mid k^{\prime}<n<k\right\} \\
& \geq \tilde{\rho}_{2 k^{\prime}+1}(s) \\
& =\rho_{2 \ell h\left(r_{k^{\prime}+1}\right)-1}(s, r, t)=\rho_{2 \ell h\left(r_{k+1}\right)-3}(s, r, t)
\end{aligned}
$$

as required. (Note $\inf \varnothing=\infty$.) 
For $t \in X_{\langle s|k, r| k\rangle}$, we complete the definition of $P_{\langle s, r, t\rangle}$ by letting $I I$ move as dictated by $\tau$, and hence by (e). Note that condition (a) continues to be satisfied.

Since $\mu_{\langle s, r\rangle}$ is $\delta^{+}$-complete, there is a set $X_{\langle s, r\rangle}$ projecting into $X_{\langle s|k, r| k\rangle}$ with $\mu_{\langle s, r\rangle}\left(X_{\langle s, r\rangle}\right)=1$ and there are $E, \rho, M, E^{\prime}, \rho^{\prime}$, and $M^{\prime}$ such that, for all $t \in X_{\langle s, r\rangle}$,

$$
\begin{gathered}
E_{2 \ell h\left(r_{k+1}\right)-2}(s, r, t)=E, \\
\rho_{2 \ell h\left(r_{k+1}\right)-2}(s, r, t)=\rho, \\
M_{2 \ell h\left(r_{k+1}\right)-1}(s, r, t)=M, \\
E_{2 \ell h\left(r_{k+1}\right)-1}(s, r, t)=E^{\prime}, \\
\rho_{2 \ell h\left(r_{k+1}\right)-1}(s, r, t)=\rho^{\prime},
\end{gathered}
$$

and

$$
M_{2 \ell h\left(r_{k+1}\right)}(s, r, t)=M^{\prime}
$$

In accordance with (f), set $\widetilde{E}_{2 k}(s)=E, \tilde{\rho}_{2 k}(s)=\rho, \widetilde{M}_{2 k+1}(s)=M, \widetilde{E}_{2 k+1}(s)=$ $E^{\prime}, \tilde{\rho}_{2 k+1}(s)=\rho^{\prime}$, and $\widetilde{M}_{2 k+2}(s)=M^{\prime}$.

It remains only to verify that $\left(\prec \mid 2 k+3,\left\langle\widetilde{M}_{k^{\prime}}(s) \mid k^{\prime} \leq 2 k+2\right\rangle,\left\langle\widetilde{E}_{k^{\prime}}(s)\right|\right.$ $\left.\left.k^{\prime}<2 k+2\right\rangle,\left\langle\tilde{\rho}_{k^{\prime}}(s) \mid k^{\prime}<2 k+2\right\rangle\right)$ is an iteration tree, and our induction step will be complete.

With $r$ as above $\left(r \supseteq r_{k+1}\right.$ and $\left.\ell h(r)=\ell h(s)\right)$, let $t \in X_{\langle s, r\rangle}$. Most of the properties of iteration trees are easily verified. Some examples:

$$
E_{2 \ell h\left(r_{k+1}\right)-2}(s, r, t) \in \widehat{M}_{2 \ell h\left(r_{k+1}\right)-2}(s, r, t),
$$

so $\widetilde{E}_{2 k}(s) \in \widetilde{M}_{2 k}(s)$. If $\operatorname{lh}\left(r_{k+1}\right)>1$ then

$$
M_{2 \ell h\left(r_{k+1}\right)-1}(s, r, t)=\operatorname{Uit}\left(M_{2 \ell h\left(r_{k+1}\right)-3}(s, r, t) ; E_{2 \ell h\left(r_{k+1}\right)-2}(s, r, t)\right),
$$

so

$$
\widetilde{M}_{2 k+1}(s)=\operatorname{Uit}\left(\widetilde{M}_{2 k^{\prime}+1}(s) ; \widetilde{E}_{2 k}(s)\right) \text {, }
$$

where $k^{\prime}<k$ is maximal such that $r_{k^{\prime}+1} \subseteq r_{k+1} .2 k^{\prime}+1$ is the $\prec$ immediate predecessor of $2 k+1$, so $\widetilde{M}_{2 k+1}(s)=\operatorname{Ult}\left(\widetilde{M}_{(2 k)^{*}}(s) ; \widetilde{E}_{2 k}(s)\right)$ as required. We omit the other verifications, except for that of $\tilde{\rho}_{2 k-1}(s) \leq \tilde{\rho}_{2 k}(s)$ for $k>0$. We have that $\tilde{\rho}_{2 k}(s)=\rho_{2 \ell h\left(r_{k+1}\right)-2}(s, r, t)$. By property (6) of pseudoalternating chains,

$$
\rho_{2 \ell h\left(r_{k+1}\right)-2}(s, r, t) \geq \operatorname{crit}\left(E_{2 \ell h\left(r_{k+1}\right)-1}(s, r, t)\right)=\kappa_{2 \ell h\left(r_{k+1}\right)-1}(s, r, t) .
$$

By $\widetilde{R} 4$,

By (c),

$$
\kappa_{\left.2 \ell h\left(r_{k+1}\right)\right)-1}(s, r, t)>\alpha_{\ell h\left(r_{k+1}\right)-1}(s, r, t) .
$$

$$
\alpha_{\ell h\left(r_{k+1}\right)-1}(s, r, t)=\tilde{\rho}_{2 k-1}(s) \text { for } k>0 .
$$


Theorem 5.10. If $T$ is a $\delta^{+}$-homogeneous tree on $(\omega \times \omega) \times \lambda$ and $\delta$ is Woodin, then $\{x \mid(\forall y)(x, y) \notin p[T]\}$ has embedding normal form.

Proof. Let $\left\langle\mu_{\langle s, r\rangle} \mid s \in{ }^{<\omega} \omega \& r \in{ }^{<\omega} \omega \& \ell h(s)=\ell h(r)\right\rangle$ witness that $T$ is $\delta^{+}$-homogeneous. By Lemma 5.8 let $\kappa_{0}<\delta$ be such that $I I$ has a winning strategy $\tau$ for $\tilde{\mathscr{G}}_{\kappa_{0}}^{T}$. Let

$$
\left\langle\mathscr{T}_{s} \mid s \in{ }^{<\omega} \omega\right\rangle, \quad\left\langle X_{\langle s, r\rangle} \mid s \in{ }^{<\omega} \omega \& r \in{ }^{<\omega} \omega \& \operatorname{lh}(s)=\operatorname{lh}(r)\right\rangle,
$$

and

$$
\left\langle P_{\langle s, r, t\rangle} \mid s \in{ }^{<\omega} \omega \& r \in{ }^{<\omega} \omega \& \operatorname{lh}(s)=\ell h(r) \& t \in X_{\langle s, r\rangle}\right\rangle
$$

be given by Lemma 5.9 .

Note that the $\delta^{+}$completeness of $\mu_{\langle s, r\rangle}$ guarantees that there is a set of $t$ of $\mu_{\langle s, r\rangle}$ measure 1 on which $u_{k}(s, r, t)$ is constant. Without loss of generality, we may assume that $X_{\langle s, r\rangle}$ is a subset of this set. Let then $u(s, r)=u(s, r, t)$ for all $t \in X_{\langle s, r\rangle}$.

Let us show that the system

$$
\left.\left(\left\langle\widetilde{M}_{2 \ell h(s)}\right| s \in{ }^{<\omega} \omega\right),\left\langle\tilde{i}_{2 \ell h\left(s_{1}\right), 2 \ell h\left(s_{2}\right)} \mid s_{1} \subseteq s_{2} \& s_{1} \in{ }^{<\omega} \omega \& s_{2} \in{ }^{<\omega} \omega\right\rangle\right)
$$

gives embedding normal form for $\{x \mid(\forall y)(x, y) \notin p[T]\}$.

Let $x \in{ }^{\omega} \omega$, in order to check property (c) in the definition of embedding normal form. Let $\widetilde{M}_{k}=\widetilde{M}_{k}(x \uparrow k), \widetilde{E}_{k}=\widetilde{E}_{k}(x \uparrow k+1)$, and $\tilde{\rho}_{k}=\rho_{k}(x \uparrow k+1)$ for $k \in \omega$. The infinite branches of the iteration tree

$$
\mathscr{T}=\left(\prec,\left\langle M_{k} \mid k \in \omega\right\rangle,\left\langle E_{k} \mid k \in \omega\right\rangle,\left\langle\rho_{k} \mid k \in \omega\right\rangle\right)
$$

are Even $=\{2 n \mid n \in \omega\}$ and, for each $y \in{ }^{\omega} \omega, b_{y}=\{0\} \cup\left\{2 n+1 \mid r_{n+1} \subseteq y\right\}$. We must show that

$$
\begin{aligned}
& (\exists y)((x, y) \in p[T]) \Rightarrow M_{\text {Even }} \text { is illfounded; } \\
& (\forall y)((x, y) \notin p[T]) \Rightarrow M_{\text {Even }} \text { is wellfounded. }
\end{aligned}
$$

Assume first that $(\exists y)((x, y) \in p[T])$. Fix $y$ such that $(x, y) \in p[T]$. By Lemma 2.1 , there is an $f: \omega \rightarrow \lambda$ such that $(\forall k)\left(f \uparrow k \in X_{\langle x|k, y| k\rangle}\right)$. For each $k$, let $q(k)$ be such that $r_{q(k)+1}=y \uparrow k+1$. Let

$$
\beta_{k+1}=\beta_{k+1}(x \uparrow q(k)+1, y \uparrow q(k)+1, f \uparrow q(k)+1) .
$$

$\widetilde{R} 5$ implies that

$$
\beta_{k+2}<\tilde{i}_{2 q(k)+2,2 q(k+1)+2}\left(\beta_{k+1}\right)
$$

where the $\tilde{i}_{m, n}, m \prec n$, are the canonical elementary embeddings associated with $\mathscr{T}$. Thus $\left\langle\tilde{i}_{2 q(k)+2 \text {,Even }}\left(\beta_{k+1}\right) \mid k \in \omega\right\rangle$ is an infinite descending sequence of ordinals of $\widetilde{M}_{\text {Even }}$.

Assume now that $(\forall y)(x, y) \notin p[T]$. $\widetilde{\mathrm{R}} 7$ gives that, for each $k \in \omega$,

$$
\left\langle\left\langle x \uparrow \ell h\left(r_{k+1}\right), r_{k+1}\right\rangle, u_{\ell h\left(r_{k+1}\right)}(x \uparrow k+1, r)\right\rangle \in\left(i_{0,2 \ell h\left(r_{k+1}\right)-1}(x \uparrow k+1, r, t)\right)(T)
$$


for all $r \supseteq r_{k+1}$ with $\ell h(r)=k+1$ and all $t \in X_{\langle x \mid k+1, r\rangle}$. Since

$$
i_{0,2 \ell h\left(r_{k+1}\right)-1}(x \uparrow k+1, r, t)=\tilde{i}_{0,2 k+1}
$$

for all such $r$ and $t$ (by (d) and (f)), it follows that

$$
\left\langle\left\langle x \uparrow \operatorname{lh}\left(r_{k+1}\right), r_{k+1}\right\rangle, u_{\ell h\left(r_{k+1}\right)}(x \uparrow k+1, r)\right\rangle \in \tilde{i}_{0,2 k+1}(T)
$$

for all $r \supseteq r_{k+1}$ with $\ell h(r)=k+1$.

Since $(\forall y)((x, y) \notin p[T])$, the tree $T(x)$ of all $\langle r, t\rangle$ such that $\langle\langle x|$ $\ell h(r), r\rangle, t\rangle \in T$ is wellfounded. Let

$$
\gamma_{k+1}=\operatorname{rank}_{i_{0.2 k+1}(T(x))}\left(\left\langle r_{k+1}, u_{\ell h\left(r_{k+1}\right)}(x \uparrow k+1, r)\right\rangle\right)
$$

for $r \supseteq r_{k+1}$ and $\ell h(r)=k+1$. Since

$$
i_{2 \ell h\left(r_{k^{\prime}+1}\right)-1,2 \ell h\left(r_{k+1}\right)-1}(x \uparrow k+1, r, t)=\tilde{i}_{2 k^{\prime}+1,2 k+1}
$$

for $r_{k^{\prime}+1} \subseteq r_{k+1} \subseteq r, \ell h(r)=k+1$, and $t \in X_{\langle x \mid k+1, r\rangle}$, we have that

$$
\tilde{i}_{2 k^{\prime}+1,2 k+1}\left(u_{k^{\prime}+1}(x \uparrow k+1, r)\right) \subsetneq u_{k+1}(x \uparrow k+1, r)
$$

for $r_{k^{\prime}+1} \subseteq r_{k+1} \subseteq r$ and $\ell h(r)=k+1$. Thus $\tilde{i}_{2 k^{\prime}+1,2 k+1}\left(\gamma_{k^{\prime}+1}\right)>\gamma_{k+1}$. If we let $\xi_{2 k+1}=\gamma_{k+1}$, then the $\xi_{n}, n \in \omega$-Even, satisfy the hypotheses of Lemma 3.2, and therefore $M_{\text {Even }}$ is wellfounded.

Theorem 5.11 (Main Theorem). If $T$ is a $\delta^{+}$-homogeneous tree on $(\omega \times \omega) \times Z$, $\delta$ is Woodin, and $\widetilde{T}$ is defined from $T$ as in $\S 2$, then $(\forall \alpha<\delta)(\widetilde{T}$ is $\alpha$ homogeneous).

Proof. We may assume $Z=\lambda$, since $\lambda$ was an arbitrary cardinal $>\delta$ and replacing $Z$ by a set of ordinals gives a tree isomorphic to the original $\tilde{T}$. Let $\left\langle\mu_{\langle s, r\rangle} \mid s \in{ }^{<\omega} \omega \& r \in{ }^{<\omega} \omega \& \ell h(s)=\ell h(r)\right\rangle$ witness that $T$ is $\delta^{+}$. homogeneous. Let $\alpha<\kappa_{0}<\delta$ be such that $I I$ has a winning strategy for $\tilde{\mathscr{G}}_{\kappa_{0}}^{T}$. Let $\widetilde{M}_{k}(s), \widetilde{E}_{k}(s), \tilde{\rho}_{k}(s)$, and $\tilde{i}_{m, n}(s)$ be as in the proof of Theorem 5.10 .

For $k<\ell h(s), r_{k} \subseteq r$, and $t \in X_{\langle s, r\rangle}$, consider the ordinal $\beta_{\ell h\left(r_{k}\right)}(s, r, t)$. By the second part of condition (a), this ordinal depends only on $s$ and $t i$ $\ell h\left(r_{k}\right)$ for $t \in X_{\langle s, r\rangle}$. For $s \in{ }^{<\omega} \omega$ and $k \leq \ell h(s)$, we may thus define $f_{k}^{s}: Z_{s}^{k} \rightarrow \mathrm{ON}$ with $\mu_{\left(\operatorname{si\ell h}\left(r_{k}\right), r_{k}\right\rangle}\left(Z_{s}^{k}\right)=1$ by

$$
f_{k}^{s}(t)=\beta_{\ell h\left(r_{k}\right)}\left(s, r, t^{\prime}\right)
$$

for all $t \in Z_{s}^{k}$, all $r \supseteq r_{k}$ with $\ell h(r)=\ell h(s)$, and all $t^{\prime} \supseteq t$ with $t^{\prime} \in X_{\langle s, r\rangle}$.

Let $e_{k}(s)=\llbracket f_{k}^{s} \rrbracket_{\mu_{\left.\left(1, r / h r_{k}\right), r_{k}\right)}}$. Define measures $\nu_{s}$ for $s \in{ }^{<\omega} \omega$ by

$$
\nu_{s}(X)=1 \Leftrightarrow\left\langle\left(\tilde{i}_{2 k, 2 \ell h(s)}(s)\right)\left(e_{k}(s)\right) \mid k<\ell h(s)\right\rangle \in\left(\tilde{i}_{0,2 \ell h(s)}(s)\right)(X) .
$$

Since the critical points of all $E_{k}\left(s^{\prime}\right)$ are $\geq \kappa_{0}$ the $\nu_{s}$ are all $\kappa_{0}$-complete and so $\alpha$-complete. If $s_{1} \subseteq s_{2}$ then $\nu_{s_{1}}(X)=1 \Leftrightarrow \nu_{s_{2}}\left(\left\{z|z| \ln \left(s_{1}\right) \in X\right\}\right)=$ 1 , exactly as in the proof of Theorem 5.6. Furthermore, if $x \in p[\widetilde{T}]$ then 
$(\forall y)((x, y) \notin p[T])$ and so the proof of Theorem 5.6 gives that the model $\widetilde{M}_{\text {Even }}(x)$ which is the direct limit of

$$
\left(\left\langle\widetilde{M}_{2 k}(x \mid k) \mid k \in \omega\right\rangle,\left\langle\tilde{i}_{2 m, 2 n}(x \uparrow n+1) \mid m \leq n \in \omega\right\rangle\right)
$$

is wellfounded. By Lemma 1.2(b) it follows that

$$
x \in p[\widetilde{T}] \Rightarrow \operatorname{Ult}\left(V ;\left\langle\nu_{x \mid n} \mid n \in \omega\right\rangle\right) \text { is wellfounded. }
$$

The theorem will be proved if we can show that, for all $s \epsilon^{<\omega} \omega, \nu_{s}\left(\widetilde{T}_{s}\right)=1$. Fix then $s \in{ }^{<\omega} \omega$. We must show that

$$
\nu_{s}\left(\left\{z \mid(\forall k)\left(\forall k^{\prime}\right)\left(\left(r_{k} \subseteq r_{k^{\prime}} \& k^{\prime}<\ell h(s)\right) \Rightarrow z\left(k^{\prime}\right)<j_{k, k^{\prime}}(z(k))\right)\right\}\right)=1 \text {, }
$$

where $j_{k, k^{\prime}}$ is the canonical elementary embedding of $\operatorname{Ult}\left(V ; \mu_{\left\langle s \mid \ell h\left(r_{k}\right), r_{k}\right\rangle}\right)$ into $\operatorname{Ult}\left(V ; \mu_{\left\langle s \mid \ell h\left(r_{k^{\prime}}\right), r_{\left.k^{\prime}\right\rangle}\right\rangle}\right)$. By the definition of $\nu_{s}$, this is equivalent to

$$
\begin{aligned}
(\forall k)\left(\forall k^{\prime}\right)\left(\left(r_{k} \subseteq r_{k^{\prime}}\right.\right. & \left.\& k^{\prime}<\ell h(s)\right) \Rightarrow \\
& \tilde{i}_{2 k^{\prime}, 2 \ell h(s)}\left(e_{k^{\prime}}\right)<\left(\tilde{i}_{0,2 \ell h(s)}\left(j_{k, k^{\prime}}\right)\right)\left(\tilde{i}_{2 k, 2 \ell h(s)}\left(e_{k}\right)\right),
\end{aligned}
$$

where we abbreviate $e_{n}(s)$ by $e_{n}$ and $\tilde{i}_{m, n}(s)$ by $\tilde{i}_{m, n}$.

Fix then $k$ and $k^{\prime}$ with $r_{k} \subseteq r_{k^{\prime}}$ and $k^{\prime}<\ell h(s)$. We must show that $\tilde{i}_{2 k^{\prime}, 2 \ell h(s)}\left(e_{k^{\prime}}\right)<\left(\tilde{i}_{0,2 \ell h(s)}\left(j_{k, k^{\prime}}\right)\right)\left(\tilde{i}_{2 k, 2 \ell h(s)}\left(e_{k}\right)\right)$. By the elementarity of $\tilde{i}_{2 k^{\prime}, 2 \ell h(s)}$, this is equivalent to

$$
e_{k^{\prime}}<\left(\tilde{i}_{0,2 k^{\prime}}\left(j_{k, k^{\prime}}\right)\right)\left(\tilde{i}_{2 k, 2 k^{\prime}}\left(e_{k}\right)\right) \text {. }
$$

Since $\mu_{\left\langle s \mid \ell h\left(r_{k}\right), r_{k}\right\rangle}$ and $\mu_{\left\langle s \mid \ell h\left(r_{k^{\prime}}\right), r_{k^{\prime}}\right\rangle}$ are $\delta^{+}$-complete, Lemma 3.3 applies. Thus $\tilde{i}_{0,2 k^{\prime}}\left(j_{k, k^{\prime}}\right) \uparrow \mathrm{ON}=j_{k, k^{\prime}} \uparrow \mathrm{ON}$. What we must show is thus finally reduced to

$$
e_{k^{\prime}}<j_{k, k^{\prime}}\left(\tilde{i}_{2 k, 2 k^{\prime}}\left(e_{k}\right)\right) \text {. }
$$

On the other hand, $\widetilde{R} 5$ gives that

$$
\beta_{\ell h\left(r_{k^{\prime}}\right)}\left(s, r, t^{*}\right)<\left(i_{2 \ell h\left(r_{k}\right), 2 \ell h\left(r_{k^{\prime}}\right)}\left(s, r, t^{*}\right)\right)\left(\beta_{\ell h\left(r_{k}\right)}\left(s, r, t^{*}\right)\right)
$$

for all $r \supseteq r_{k+1}$ with $\ell h(r)=\ell h(s)$ and all $t^{*} \in X_{\langle s, r\rangle}$. (d) and (f) thus give that

$$
\beta_{\ell h\left(r_{k^{\prime}}\right)}\left(s, r, t^{*}\right)<\tilde{i}_{2 k, 2 k^{\prime}}\left(\beta_{\ell h\left(r_{k}\right)}\left(s, r, t^{*}\right)\right)
$$

for all such $r$ and $t^{*}$. By the definition of $f_{k}^{s}$ and $f_{k^{\prime}}^{s}$,

$$
f_{k^{\prime}}^{s}\left(t^{\prime}\right)<\tilde{i}_{2 k, 2 k^{\prime}}\left(f_{k}^{s}(t)\right)
$$

for all $t^{\prime} \in Z_{s}^{k^{\prime}}$ and all $t \in Z_{s}^{k}$ with $t \subseteq t^{\prime}$. Thus

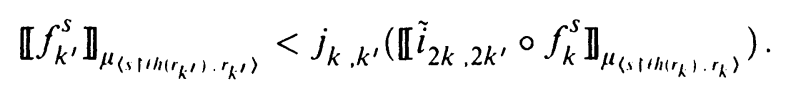

Hence

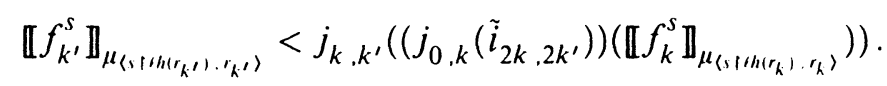


By the definitions of $e_{k}$ and $e_{k^{\prime}}$, this means that

$$
e_{k^{\prime}}<j_{k, k^{\prime}}\left(\left(j_{0, k}\left(\tilde{i}_{2 k, 2 k^{\prime}}\right)\right)\left(e_{k}\right)\right) \text {. }
$$

Applying Lemma 3.3 again, we get

$$
e_{k^{\prime}}<j_{k, k^{\prime}}\left(\tilde{i}_{2 k, 2 k^{\prime}}\left(e_{k}\right)\right) \text {. }
$$

Since this is just what we are trying to show, the proof is complete.

\section{REFERENCES}

1. A. Dodd, The core model, London Math. Soc. Lecture Notes, no. 61, Cambridge Univ. Press, Cambridge, England, 1982.

2. M. Foreman, M. Magidor, and S. Shelah, Martin's maximum, saturated ideals, and non-regular ultrafilters, Annals of Math. 127 (1988), $1-47$.

3. T. Jech, Set theory, Academic Press, New York, 1978.

4. A. Kechris, Homogeneous trees and projective scales, Cabal Seminar 1977-79 (A. S. Kechris, D. A. Martin, and Y. N. Moschovakis, eds.), Lecture Notes in Math., no. 839, Springer-Verlag, Berlin, 1981, pp. 33-74.

5. A. Levy, Axiom schemata of strong infinity in axiomatic set theory, Pacific J. Math. 10 (1960), 223-238.

6. __ Definability in axiomatic set theory II, Mathematical Logic and Foundations of Set Theory, proceedings of an International Colloquium held in Jerusalem in 1968, North-Holland, Amsterdam, 1970, pp. 129-145.

7. D. A. Martin, Measurable cardinals and analytic games, Fund. Math. 66 (1970), 287-291.

8. __ Infinite games, Proc. Internat. Congr. Math., 1978 (O. Lehto, ed.), Academia Scientiarum Fennica, Helsinki, 1980, pp. 269-273.

9. D. A. Martin and R. M. Solovay, A basis theorem for $\Sigma_{3}^{1}$ sets of reals, Ann. of Math. 89 (1969), 138-159.

10. D. A. Martin and J. R. Steel, Projective determinacy, Proc. Nat. Acad. Sci. U.S.A. (to appear).

11. Iteration trees (to appear).

12. Y. N. Moschovakis, Descriptive set theory, North-Holland, Amsterdam, 1980.

13. H. Woodin, Supercompact cardinals, sets of reals and weakly homogeneous trees, Proc. Nat. Acad. Sci. U.S.A. (to appear).

Department of Mathematics, University of California at los Angeles, Los Angeles, CALIFORNIA 90024-1555 